\title{
Seeking fixed points in multiple coupling scalar theories in the $\varepsilon$ expansion
}

\author{
Hugh Osborn ${ }^{a}$ and Andreas Stergiou ${ }^{b}$ \\ ${ }^{a}$ Department of Applied Mathematics and Theoretical Physics, \\ Wilberforce Road, Cambridge CB3 OWA, U.K. \\ ${ }^{b}$ Theoretical Physics Department, CERN, \\ Geneva, Switzerland \\ E-mail: h.osborn@damtp.cam.ac.uk, andreas.stergiou@cern.ch
}

ABSTRACT: Fixed points for scalar theories in $4-\varepsilon, 6-\varepsilon$ and $3-\varepsilon$ dimensions are discussed. It is shown how a large range of known fixed points for the four dimensional case can be obtained by using a general framework with two couplings. The original maximal symmetry, $O(N)$, is broken to various subgroups, both discrete and continuous. A similar discussion is applied to the six dimensional case. Perturbative applications of the $a$-theorem are used to help classify potential fixed points. At lowest order in the $\varepsilon$ expansion it is shown that at fixed points there is a lower bound for $a$ which is saturated at bifurcation points.

KEYwords: Conformal Field Theory, Renormalization Group

ARXIV EPRINT: 1707.06165 


\section{Contents}

1 Introduction 1

2 Two flavour scalar theory in $6-\varepsilon$ dimensions $\quad 6$

3 Two flavour scalar theory in $4-\varepsilon$ dimensions $\quad 10$

4 Scalar theories with reduced symetry in $6-\varepsilon$ dimensions $\quad 14$

$\begin{array}{lll}4.1 & O(m) \times O(n) \text { theories in } 6-\varepsilon \text { dimensions } & 19\end{array}$

5 Scalar theories with reduced symmetry in $4-\varepsilon$ dimensions $\quad 23$

$\begin{array}{lll}5.1 & \text { Fixed points with discrete } H \subset O(N) & 29\end{array}$

$\begin{array}{lll}5.1 .1 & \text { Hypercubic fixed poins } & 29\end{array}$

5.1.2 Hypertetrahedral fixed points 31

5.2 Fixed points with continuous $H \subset O(N)$

$\begin{array}{lll}5.2 .1 & O(m) \times O(n) \text { fixed points } & 36\end{array}$

$\begin{array}{lll}\text { 5.2.2 } & M N \text { fixed points } & 39\end{array}$

6 Scalar theories in $3-\varepsilon$ dimensions $\quad 40$

7 Multiple couplings in $4-\varepsilon$ dimensions $\quad 47$

8 Conclusion $\quad 53$

A Bifurcations of fixed points in $6-\varepsilon$ dimensions $\quad 54$

B Alternative fixed points in $4-\varepsilon$ dimensions $\quad 55$

$\begin{array}{ll}\text { C Bounds for tensor products } & 57\end{array}$

D Perturbations at the hypertetrahedral fixed point $\quad 59$

$\begin{array}{lll}\mathrm{E} C_{T} \text { for scalar theories } & 61\end{array}$

\section{Introduction}

In seeking conformal field theories, whether through finding zeros of $\beta$-functions or applying bootstrap consistency conditions, it is natural to impose as much symmetry as possible. This then restricts the number of couplings and correspondingly non zero operator product coefficients so that the number of channels to be considered in a bootstrap analysis is reduced. In the cardinal exemplar of the success of the numerical bootstrap, the three dimensional Ising model with two relevant fields $\sigma, \epsilon$, there is a $\mathbb{Z}_{2}$ symmetry with $\sigma \mathbb{Z}_{2}$ odd. 
The imposition of symmetries might introduce a selection bias in finding non trivial CFTs. On the other hand the fixed points of non symmetric theories might have an emergent symmetry. According to the notion of universality fixed points are determined by the associated symmetry group. To an extent it is often tacitly assumed that fixed points without a high degree of symmetry may be neglected. Nevertheless for any potential classification of CFTs in three or more dimensions it is necessary to determine all possible fixed points which may have smaller symmetry groups than have been hitherto considered. Even with knowledge of the fixed points in quantum field theories in particular dimensions, an important question is how they are linked under possible RG flows induced by perturbations by adding relevant operators. Determining the spectrum of relevant operators is part of the data defining a CFT or can be found by solving linear eigenvalue equations. What happens under RG flow, and whether new fixed points are attained, is a non linear problem. For slightly relevant perturbations it is possible to use conformal perturbation theory but this becomes difficult beyond lowest order. In a perturbative context the $\varepsilon$ expansion can be taken to higher order by determining $\beta$-functions and anomalous dimensions in a time honoured fashion. This may be applied, as originally suggested by Wilson and Fisher [1], in $4-\varepsilon$ dimensions but can also be used in $6-\varepsilon$ and $3-\varepsilon$ dimensions.

In two dimensions, at least for minimal models, there is a well understood framework of CFTs which are linked by RG flows. In two dimensions conformal perturbation theory can be used systematically, as originally proposed by Zamolodchikov [2] and extended subsequently [3-8]. A crucial organising principle is the $c$-theorem which requires that the Virasoro central charge $c$ for any unitary CFT at fixed points reached by RG flow arising from a relevant perturbation has a lower value of $c$ than the original unperturbed CFT. Minimal models, with $c<1$, then flow ultimately to the Ising model with $c=\frac{1}{2}$.

There is no such simple picture in three or other dimensions of current interest. Here we attempt to discuss potential fixed points and RG flows using the $\varepsilon$-expansion with various additional assumptions for simplicity. For determining critical exponents in many different condensed matter systems with a wide range of symmetries there is a large literature from the 1970's and later. A review discussing large number such models which are applicable to critical phenomena for condensed matter systems is [9]. The $\varepsilon$-expansion is a version of conformal perturbation theory starting from free field theories and which allows an extension beyond lowest order. An assumption made here is that it is qualitatively correct in determining possible CFTs in three dimensions starting from $4-\varepsilon$ dimensions through essentially one loop calculations. Of course in particular applications loop calculations to three or more loops and sophisticated resummation methods may be used to obtain more accurate results for critical exponents.

Results in the $\varepsilon$-expansion depend critically on the number $\mathcal{N}$ of scalar fields. The symmetry of the kinetic term is $O(\mathcal{N})$. In $4-\varepsilon$ dimensions there is a fixed point with $O(N)$, $N=\mathcal{N}$ symmetry. This is stable for $N \lesssim 4$. For $N>4$ there are relevant perturbations which lead to a variety of fixed points. Whether there are fixed points which cannot be reached by simple $\mathrm{RG}$ flows from free fields is not yet clear. A related question is how many couplings are necessary in order to find particular fixed points. For the Ising model it is sufficient to consider just $\mathcal{N}=1$ and the $\phi^{4}$ perturbation with a single coupling. In $6-\varepsilon$ 
dimensions if $\mathcal{N}=N+1$ an $O(N)$ renormalisable theory is formed by fields forming an $N$ vector and a singlet. There are then fixed points with $O(N)$ symmetry if $N$ is large enough.

In $\varepsilon$-expansions the starting point is a potential of the form

$$
V(\phi ; g)=\sum_{I} g^{I} P_{I}(\phi),
$$

where $\left\{P_{I}(\phi)\right\}$ are polynomials of degree 4 , or 3 or 6 , depending on the dimension. The symmetry group $H_{g}$ for $V$ is determined by

$$
V(\mathrm{~g} \phi ; g)=V(\phi ; g) \text { for all } \mathrm{g} \in H_{g} \subset O(N) .
$$

For each polynomial $P_{I}(\phi)$ there is a corresponding symmetry group $H_{I}$ defined as in (1.2). For generic couplings $H_{g} \supseteq H \simeq \cap H_{I}$, but $H_{g}$ may be larger than $H$. Under RG flow the symmetry is in general $\cap H_{g}$ for all $g$, but the symmetry may be enhanced at particular points in the space of couplings. It is also important to note that the larger symmetry defined by

$$
V(\mathrm{~g} \phi ; g)=V(\phi ; g \mathrm{~g}), \quad(g \mathrm{~g})^{I}=g^{J} \mathrm{~g}_{J}^{I}, \quad \text { for all } \mathrm{g} \in G_{g} \subset O(N),
$$

defines a physically equivalent theory if $(g \mathrm{~g})^{I} \neq g^{I}$. At a fixed point with $g=g_{*}$ if $G_{g_{*}} / H_{g_{*}}$ is discrete then there are equivalent fixed points with identical critical exponents. If $G_{g_{*}} / H_{g_{*}}$ is continuous there are submanifolds of equivalent fixed points which contain apparently exactly marginal operators. In general $G_{g} \supset G$ where $G \subset O(N)$ is the symmetry associated to the set of polynomials $\left\{P_{I}\right\}$ such that for $\mathrm{g} \in G, P_{I}(\mathrm{~g} \phi)=\mathrm{g}_{I}^{J} P_{J}(\phi)$. Of course if $\left\{P_{I}\right\}$ includes all $\left(\begin{array}{c}N+p \\ p\end{array}\right)$ polynomials of degree $p$ then $G \simeq O(N)$, this is still true if $\left\{P_{I}\right\}$ forms a representation space for a faithful irreducible representation of $O(N)$.

Determining all possible polynomials invariant under subgroups of $O(N)$, discrete or continuous, is very non trivial task. For $N=2,3$ the results are simple but general results for quartic polynomials when $N=4[10,11]$ or $N=6$ are much more involved [12-14]. An important result due to Michel [15] in $4-\varepsilon$ dimensions is that stable fixed points are unique (an essentially identical proof is described in [16]). This then implies at such a fixed point $G_{g_{*}} \simeq H_{g_{*}}$. This does not hold if there is a submanifold of continuously connected equivalent fixed points but otherwise provides constraints on the possible existence of stable fixed points for particular breakings of $O(N)$, or in $6-\varepsilon$ dimensions.

RG flow for multiple couplings, which is determined by solving

$$
\frac{\mathrm{d}}{\mathrm{d} t} g^{I}=-\beta^{I}(g)
$$

is potentially very non trivial but is constrained by the $a$-theorem in four dimensions and its possible extensions in other cases. In a strong version of the $a$-theorem there exists a function $a(g)$ which decreases monotonically under RG flow until the flow reaches fixed points $g^{I}=g_{*}^{I}$ where the $\beta$-functions vanish. For a stable fixed point $a(g)$ has a local minimum and the stability matrix $\left[M_{I}^{J}\right]$ defined by

$$
M_{I}^{J}=\left.\partial_{I} \beta^{J}(g)\right|_{g=g_{*}},
$$


is positive definite. In four dimensions an $a$-function satisfying the requirements for a strong $a$-theorem can be constructed directly from the one and two loop $\beta$-functions [17], the same is true in six dimensions [18] and, as will be shown later, in three dimensions. In four [19] and six [20] there are arguments that these results, expressing the $\beta$-functions as a gradient flow, can be extended to arbitrary perturbative order. This imposes non trivial constraints on the higher loop $\beta$-functions, even for purely scalar theories. Using minimal subtraction the expressions for $a(g)$ can be easily extended to $4-\varepsilon$ and $6-\varepsilon$ dimensions and so the existence of an $a$-function is relevant for fixed points accessible through an $\varepsilon$ expansion. There are however no comparable arguments for theories starting from three dimensions. In four dimensions at a fixed point $a$, determined by the trace anomaly for a curved background or the three point function for the energy momentum tensor on flat space, is necessarily positive. However in a perturbative discussion in $4-\varepsilon$ dimensions the results for $a(g)$ away from fixed points are not necessarily bounded below. Under RG flow $a(g)$ decreases but it may be that no fixed point is attained and the assumption that the couplings are $\mathrm{O}(\varepsilon)$ breaks down. In particular $a(g)$ could become negative in situations where the potential $V(\phi ; g)$ is also unbounded below and there is no stable ground state. These conclusions hold even more strongly in perturbative discussions in $6-\varepsilon$ dimensions, since cubic potentials of course are never bounded below.

In our discussions in $6-\varepsilon$ and $4-\varepsilon$ dimensions we consider perturbative expressions for $a(g)$, neglecting the free field contributions which are irrelevant here and also allowing for convenience an arbitrary overall normalisation. The most stable fixed point $g_{*}$ is then the one at which $a\left(g_{*}\right)$ is the least local minimum. In the framework of the $\varepsilon$-expansion we are able to show that $a\left(g_{*}\right)$ has a lower bound, of order $\varepsilon^{2}, \varepsilon^{3}$ in $6-\varepsilon, 4-\varepsilon$ dimensions. This bound is attained when two fixed points collide and then disappear from the set of fixed points for real couplings, so that there is a saddle-node bifurcation. If the initial couplings are small but are such that $a(g)$ is less than the bound then the RG flow cannot reach perturbative fixed points calculable in the $\varepsilon$-expansion. The existence of $a(g)$ nevertheless avoids the possibility of more complicated bifurcations [21].

In this paper we discuss a range of fixed points present in scalar field theories when the number of fields $\mathcal{N}>1$ and there are two or more couplings. For simplicity we first consider in sections 2 and 3 the cases when $\mathcal{N}=2$ in $6-\varepsilon$ and $4-\varepsilon$ dimensions. For an arbitrary renormalisable theory a complete analysis of potential fixed points is possible. In the four dimensional case there is just the $O(2)$ symmetric point and two decoupled Ising fixed points. In both cases the couplings transform under $O(2)$ and potential fixed points are related to the different irreducible components.

For general $N$ there are many more possibilities of finding fixed points where the initial symmetry is broken by slightly relevant perturbations. In section 4 we consider perturbations of the $O(N)$ symmetric theory in six dimensions. The unperturbed theory has a critical point in $6-\varepsilon$ dimensions so long as $N>N_{\text {crit }}[22,23]$. With one additional coupling for a relevant operator there is a breaking $O(N) \rightarrow O(m) \times O(n), m+n=N$. There is then a new fixed point corresponding to an $O(m)$ invariant theory with $n$ decoupled free fields so long as $m>N_{\text {crit }}$, or equivalently $m \leftrightarrow n$. We also consider breakings $O(N) \rightarrow O(m) \times O(n)$, $m n=N$ involving additional fields which form symmetric traceless tensor under $O(m)$. 
In section 5 we analyse breakings of the $O(N)$ symmetric theory in $4-\varepsilon$ dimensions due to perturbations formed by a symmetric traceless 4-tensor. At lowest order in the $\varepsilon$-expansion this becomes relevant for $N>4$. We consider a general framework in which the $\mathrm{RG}$ flow may be restricted to a two dimensional space of couplings. In the simplest case to three loops there are three coefficients $a, b, c$, but overall normalisation is arbitrary and there are bounds which constrain the allowed values. This formalism covers a range of theories considered in the literature, including ones with hypercubic and hypertetrahedral symmetry, and also cases where there is an unbroken $O(m)$ symmetry, $N=n m$. It is shown how the $a$-function has a lower bound at fixed points which is attained at bifurcation points where new fixed points emerge. The general formalism is shown to give identical results to three loops with previous calculations for particular models.

We also discuss in section 6 scalar theories in $3-\varepsilon$ dimensions with a $\phi^{6}$ interaction using results for $\beta$-functions for a general renormalisable potential at two and four loops. Such theories have an $O(N)$ invariant fixed point but, by determining the anomalous dimensions of scalar operators with dimensions less than three, there is no apparent large $N$ theory valid for dimensions away from three (the $O(N)$ theories in four and six dimensions at large $N$ can be related to an $O(N)$ model for any $d$ with an interaction $\sigma \phi^{2}$ ). The $O(N)$ fixed point is unstable against perturbations for any $N$ and we consider the simple case leading to a fixed point with hypercubic symmetry.

In section 7 we extend the framework of section 5 to multiple couplings corresponding to several slightly relevant operators formed by symmetric traceless 4 -tensors. The algebraic conditions necessary for a closed RG flow are generalised to this case and it is shown how the bound on the $a$-function remains valid to lowest order in $\varepsilon$ and that this bound is realised at the bifurcation point where there is an exactly marginal operator. An example with three couplings, in which there are five inequivalent fixed points, is considered. A theory with arbitrarily many couplings with a closed RG flow is also considered.

Various details are contained in five appendices. In appendix A we analyse the bifurcation point for the $O(N)$ symmetric theory in $6-\varepsilon$ dimensions. Appendix B describes a different $4-\varepsilon$ fixed point than the ones considered in section 5 . In appendix $\mathrm{C}$ we obtain bounds on the coefficients $a, b$ which appear in the general discussion in $4-\varepsilon$ dimensions. The bounds are saturated in the case of hypercubic symmetry. Appendix D considers perturbations of the theory with hypertetrahedral symmetry. The analysis shows in general the presence of relevant operators. Finally in appendix $\mathrm{E}$ we show how the perturbative expression for $C_{T}$, the coefficient of the energy momentum tensor two point function, at the fixed point can be constrained by large $N$ results. This allows $C_{T}$ to be found for arbitrary theories in the $\varepsilon$ expansion in $4-\varepsilon$ dimensions to order $\varepsilon^{3}$.

In general the different sections are more or less independent and may be read, if desired, separately. Detailed results for many cases have already appeared in the literature but are here presented in a hopefully unified framework. 


\section{Two flavour scalar theory in $6-\varepsilon$ dimensions}

A general two component $\phi^{3}$ theory is described by a potential

$$
V(\phi)=\frac{1}{6} \lambda_{1} \phi_{1}^{3}+\frac{1}{6} \lambda_{2} \phi_{2}^{3}+\frac{1}{2} g_{1} \phi_{1} \phi_{2}^{2}+\frac{1}{2} g_{2} \phi_{1}^{2} \phi_{2},
$$

depending on couplings $G_{I}=\left(\lambda_{1}, \lambda_{2}, g_{1}, g_{2}\right)$. Results for $\beta$-functions for such renormalisable $\phi^{3}$ theories, with fields $\phi_{i}$, in $6-\varepsilon$ dimensions are succinctly given in terms of $\beta_{V}(\phi)$ where, taking $V \rightarrow(4 \pi)^{\frac{3}{2}} V$,

$$
\beta_{V}(\phi)=\varepsilon V(\phi)+V_{i}(\phi)\left(-\frac{1}{2} \varepsilon \delta_{i j}+\gamma_{i j}\right) \phi_{j}+\bar{\beta}_{V}(\phi), \quad V_{i}=\partial_{i} V
$$

for $\gamma_{i j}$ the symmetric anomalous dimension matrix and $\bar{\beta}_{V}$ corresponding to the contributions of one particle irreducible graphs. Taking $V(\phi)=\frac{1}{6} \lambda_{i j k} \phi_{i} \phi_{j} \phi_{k}$ then $\beta_{V}(\phi)=$ $\frac{1}{6} \beta_{i j k} \phi_{i} \phi_{j} \phi_{k}$. At one loop

$$
\bar{\beta}_{V}^{(1)}=-\frac{1}{6} V_{i j} V_{j k} V_{k i}, \quad \gamma_{i j}^{(1)} \phi_{i} \phi_{j}=\frac{1}{12} V_{i j} V_{i j},
$$

and at two loops ${ }^{1}$

$$
\begin{aligned}
\bar{\beta}_{V}(2) & =-\frac{1}{8} V_{i j} V_{i k} V_{l m} \lambda_{j l n} \lambda_{k m n}+\frac{7}{144} V_{i j} V_{j k}\left(\lambda^{2}\right)_{k l} V_{l i}-\frac{1}{12} V_{i l} V_{j m} V_{k n} \lambda_{i j k} \lambda_{l m n}, \\
\gamma_{i j}{ }^{(2)} \phi_{i} \phi_{j} & =\frac{1}{18}\left(V_{i j} V_{k l} \lambda_{i k m} \lambda_{j l m}-\frac{11}{24} V_{i j}\left(\lambda^{2}\right)_{j k} V_{i k}\right), \quad\left(\lambda^{2}\right)_{i j}=\lambda_{i k l} \lambda_{j k l}, \quad \lambda_{i j k}=V_{i j k} .
\end{aligned}
$$

and also at three loops

$$
\begin{aligned}
\bar{\beta}_{V}{ }^{(3)}= & \frac{1}{6}\left(-V_{i j} V_{k l} V_{m n}\left(\lambda_{i k p} \lambda_{j m q} \lambda_{l n r} \lambda_{p q r}+\frac{1}{24}\left(23-24 \zeta_{3}\right) \lambda_{i p q} \lambda_{k p r} \lambda_{m q r} \lambda_{j l n}\right)\right. \\
& -V_{i j} V_{k l}\left(\frac{9}{8} \delta_{i k} \lambda_{j r p} \lambda_{l r q}+\frac{47}{288}\left(\lambda^{2}\right)_{i k} \delta_{j p} \delta_{l q}+\frac{47}{144} \delta_{i k}\left(\lambda^{2}\right)_{j p} \delta_{l q}+\frac{3}{16} \lambda_{i k p} \lambda_{j l q}\right) \lambda_{p s m} \lambda_{q s n} V_{m n} \\
& +V_{i j} V_{j k} V_{k l}\left(\frac{23}{96} \lambda_{i m n} \lambda_{m p r} \lambda_{n q r} \lambda_{l p q}-\frac{19}{108} \lambda_{i m p} \lambda_{l m q}\left(\lambda^{2}\right)_{p q}+\frac{11}{576}\left(\lambda^{2}\right)_{i m}\left(\lambda^{2}\right)_{m l}\right) \\
& +V_{i j} V_{i k} V_{m n}\left(\frac{11}{576}\left(\lambda^{2}\right)_{j m}\left(\lambda^{2}\right)_{k n}+\frac{5}{9} \lambda_{j p q} \lambda_{k p n}\left(\lambda^{2}\right)_{q m}+\frac{3}{4} \lambda_{j p q} \lambda_{k r n} \lambda_{q s m} \lambda_{p r s}\right) \\
& +V_{i j} V_{k l} V_{m n}\left(\frac{15}{16} \lambda_{j p r} \lambda_{i r m} \lambda_{k p s} \lambda_{l s n}+\frac{11}{72} \delta_{j k} \lambda_{i p m} \lambda_{l q n}\left(\lambda^{2}\right)_{p q}+\frac{11}{48} \lambda_{i k p} \lambda_{j l n}\left(\lambda^{2}\right)_{p m}\right) \\
& \left.+V_{i j} V_{k l} V_{m n}\left(\left(1-3 \zeta_{3}\right) \lambda_{j p q} \lambda_{k p r} \lambda_{l n q} \lambda_{i m r}-\frac{1}{16}\left(29-24 \zeta_{3}\right) \delta_{j k} \lambda_{i p r} \lambda_{l q s} \lambda_{p q m} \lambda_{r s n}\right)\right), \\
\gamma_{i j}{ }^{(3)} \phi_{i} \phi_{j}= & \frac{1}{108} V_{i j} V_{k l} c_{i j k l}, \\
c_{i j k l}= & \frac{71}{16} \lambda_{i m n} \lambda_{j m p} \lambda_{n q k} \lambda_{p q l}+\frac{7}{8} \lambda_{i n p} \lambda_{j l m} \lambda_{k n q} \lambda_{m p q}-\frac{103}{96} \lambda_{i k m} \lambda_{j l n}\left(\lambda^{2}\right)_{m n} \\
& -2 \lambda_{k m n} \lambda_{j l m}\left(\lambda^{2}\right)_{i n}-\frac{121}{48} \delta_{j l} \lambda_{i m n} \lambda_{p q k} \lambda_{m p r} \lambda_{n q r}+\frac{23}{576}\left(\lambda^{2}\right)_{i k}\left(\lambda^{2}\right)_{j l} \\
& -\frac{13}{288} \delta_{j l}\left(\lambda^{2}\right)_{i m}\left(\lambda^{2}\right)_{m k}+\frac{103}{72} \delta_{j l} \lambda_{i m p} \lambda_{k n p}\left(\lambda^{2}\right)_{m n}+\frac{9}{2}\left(\frac{7}{4}-\zeta_{3}\right) \lambda_{i m n} \lambda_{j p q} \lambda_{m q k} \lambda_{n p l} .
\end{aligned}
$$

\footnotetext{
${ }^{1}$ These expressions can be obtained from results in $[18,23-25]$.
} 
Including $\mathrm{O}\left(\phi^{2}\right)$ contributions in $V(\phi)$ then results for (2.2) immediately determine the associated anomalous dimensions for such operators.

For the two component theory determined by (2.1) the maximal symmetry is determined by the transformations

$$
\phi_{\theta, 1}=\cos \theta \phi_{1}+\sin \theta \phi_{2}, \quad \phi_{\theta, 2}=-\sin \theta \phi_{1}+\cos \theta \phi_{2} .
$$

Then

$$
V_{\theta}(\phi)=V\left(\phi_{\theta}\right)
$$

with $V_{\theta}$ expressed in terms of transformed couplings

$$
G_{\theta, I}=R_{I J}(\theta) G_{J},
$$

where

$$
\left[R_{I J}(\theta)\right]=\left(\begin{array}{cccc}
\cos ^{3} \theta & -\sin ^{3} \theta & 3 \cos \theta \sin ^{2} \theta & -3 \cos ^{2} \theta \sin \theta \\
\sin ^{3} \theta & \cos ^{3} \theta & 3 \cos ^{2} \theta \sin \theta & 3 \cos \theta \sin ^{2} \theta \\
\cos \theta \sin ^{2} \theta & -\cos ^{2} \theta \sin \theta & \cos ^{3} \theta-2 \cos \theta \sin ^{2} \theta & -\sin ^{3} \theta+2 \cos ^{2} \theta \sin \theta \\
\cos ^{2} \theta \sin \theta & \cos \theta \sin ^{2} \theta & \sin ^{3} \theta-2 \cos ^{2} \theta \sin \theta & \cos ^{3} \theta-2 \cos \theta \sin ^{2} \theta
\end{array}\right)
$$

defines an equivalent theory, related by a field redefinition. The $\mathrm{SO}(2)$ transformations of the couplings given by (2.8) and (2.9) may be extended to $O(2)$ by including reflections generated by $\phi_{1} \leftrightarrow \phi_{2}$ which give an equivalence under $\lambda_{1} \leftrightarrow \lambda_{2}, g_{1} \leftrightarrow g_{2}$.

The couplings may be decomposed in terms of real two-dimensional $\mathrm{SO}(2)$ irreducible representations

$$
v_{-3}=\left(\begin{array}{c}
\lambda_{1}-3 g_{1} \\
\lambda_{2}-3 g_{2}
\end{array}\right), \quad v_{1}=\left(\begin{array}{c}
\lambda_{1}+g_{1} \\
\lambda_{2}+g_{2}
\end{array}\right),
$$

where under $G_{I} \rightarrow G_{\theta, I}$

$$
v_{n} \rightarrow R_{n}(\theta) v_{n}, \quad R_{n}(\theta)=\left(\begin{array}{cc}
\cos n \theta-\sin n \theta \\
\sin n \theta & \cos n \theta
\end{array}\right) .
$$

The four couplings can be reduced to three invariants

$$
\begin{aligned}
I_{1} & =\left(\lambda_{1}-3 g_{1}\right)^{2}+\left(\lambda_{2}-3 g_{2}\right)^{2}, \quad I_{2}=\left(\lambda_{1}+g_{1}\right)^{2}+\left(\lambda_{2}+g_{2}\right)^{2}, \\
I_{3}+i I_{4} & =\left(\left(\lambda_{1}+g_{1}\right)+i\left(\lambda_{2}+g_{2}\right)\right)^{3}\left(\left(\lambda_{1}-3 g_{1}\right)+i\left(\lambda_{2}-3 g_{2}\right)\right),
\end{aligned}
$$

where $I_{3}^{2}+I_{4}^{2}=I_{1} I_{2}^{3}$. Clearly $I_{4}$ is odd under $\lambda_{1} \leftrightarrow \lambda_{2}, g_{1} \leftrightarrow g_{2}$. The invariants determine the couplings up to an $\mathrm{SO}(2)$ equivalence.

From (3.9) invariant subspaces under $\mathrm{SO}(2)$ are obtained by imposing either $v_{1}$ or $v_{-3}$ to be zero giving the conditions

$$
\text { a) } \lambda_{1}=-g_{1}, \lambda_{2}=-g_{2} \text { and b) } \lambda_{1}=3 g_{1}, \lambda_{2}=3 g_{2} \text {. }
$$

Case a) corresponds to $\lambda_{i j k}$ in (2.1) being symmetric traceless and case b) to taking $\lambda_{i j k}=$ $3 \delta_{(i j} v_{k)}$ with $v_{i}=\left(g_{1}, g_{2}\right)$. With the restrictions in (2.13) the only invariants are $I_{1}$ or $I_{2}$ 
respectively. If $\lambda_{2}=g_{2}=0$ and $g_{1}=-\lambda_{1}$ then the $O(2)$ symmetry is reduced to $\mathbb{Z}_{3}$; in this case $V(\phi)=\frac{1}{12} \lambda_{1}\left(\left(\phi_{1}+i \phi_{2}\right)^{3}+\left(\phi_{1}-i \phi_{2}\right)^{3}\right)$. If $g_{1, \theta}=g_{2, \theta}=0$ for some $\theta$, so that the theory is equivalent to two decoupled theories, then it is necessary that $I_{1}=I_{2}$, or

$$
g_{1}^{2}+g_{2}^{2}=\lambda_{1} g_{1}+\lambda_{2} g_{2} .
$$

The symmetric $2 \times 2$ anomalous dimension matrix for this theory is $\gamma(G)$ and transforms as

$$
\gamma\left(G_{\theta}\right)=R_{1}(\theta) \gamma(G) R_{1}(\theta)^{T} .
$$

Hence $\operatorname{tr} \gamma$, $\operatorname{det} \gamma$, and the eigenvalues of $\gamma$, are scalars under the equivalence transformations of the couplings and are functions of the invariants $I_{1,2,3}$. For the $\beta$-function

$$
\beta_{I}\left(G_{\theta}\right)=R_{I J}(\theta) \beta_{J}(G) .
$$

This ensures that for a fixed point when $\beta_{I}\left(G_{*}\right)=0$ then also $\beta_{I}\left(G_{* \theta}\right)=0$.

For the theory defined by (2.1) the $\beta$-functions to one loop are given from (2.3):

$$
\begin{aligned}
\beta_{\lambda_{1}}{ }^{(1)}= & -\frac{1}{2} \varepsilon \lambda_{1}-\lambda_{1}^{3}-g_{1}^{3}-3\left(\lambda_{1}+g_{1}\right) g_{2}^{2} \\
& +\frac{1}{4}\left(\lambda_{1}\left(\lambda_{1}^{2}+g_{1}^{2}+2 g_{2}^{2}\right)+g_{2}\left(\lambda_{1} g_{2}+\lambda_{2} g_{1}+2 g_{1} g_{2}\right)\right), \\
\beta_{\lambda_{2}}{ }^{(1)}= & -\frac{1}{2} \varepsilon \lambda_{2}-\lambda_{2}^{3}-g_{2}^{3}-3\left(\lambda_{2}+g_{2}\right) g_{1}^{2} \\
& +\frac{1}{4}\left(\lambda_{2}\left(\lambda_{2}^{2}+2 g_{1}^{2}+g_{2}^{2}\right)+g_{1}\left(\lambda_{1} g_{2}+\lambda_{2} g_{1}+2 g_{1} g_{2}\right)\right), \\
\beta_{g_{1}}{ }^{(1)}= & -\frac{1}{2} \varepsilon g_{1}-\left(\lambda_{1}+g_{1}\right)\left(g_{1}^{2}+g_{2}^{2}\right)-g_{1}\left(\lambda_{2}+g_{2}\right)^{2} \\
& +\frac{1}{12}\left(g_{1}\left(\lambda_{1}^{2}+2 \lambda_{2}^{2}+5 g_{1}^{2}+4 g_{2}^{2}\right)+\left(\lambda_{2}+2 g_{2}\right)\left(\lambda_{1} g_{2}+\lambda_{2} g_{1}+2 g_{1} g_{2}\right)\right), \\
\beta_{g_{2}}{ }^{(1)}= & -\frac{1}{2} \varepsilon g_{2}-\left(\lambda_{2}+g_{2}\right)\left(g_{1}^{2}+g_{2}^{2}\right)-g_{2}\left(\lambda_{1}+g_{1}\right)^{2} \\
& +\frac{1}{12}\left(g_{2}\left(2 \lambda_{1}^{2}+\lambda_{2}^{2}+4 g_{1}^{2}+5 g_{2}^{2}\right)+\left(\lambda_{1}+2 g_{1}\right)\left(\lambda_{1} g_{2}+\lambda_{2} g_{1}+2 g_{1} g_{2}\right)\right) .
\end{aligned}
$$

The positive contributions in the second line of each expression arise from the $2 \times 2$ anomalous dimension matrix which at lowest order is, from (2.3),

$$
\gamma^{(1)}=\frac{1}{12}\left(\begin{array}{cc}
\lambda_{1}^{2}+g_{1}^{2}+2 g_{2}^{2} & \lambda_{1} g_{2}+\lambda_{2} g_{1}+2 g_{1} g_{2} \\
\lambda_{1} g_{2}+\lambda_{2} g_{1}+2 g_{1} g_{2} & \lambda_{2}^{2}+2 g_{1}^{2}+g_{2}^{2}
\end{array}\right) .
$$

Finding zeros of the $\beta$-functions giving fixed points requires relations between the couplings. Requiring that the couplings satisfy a) in (2.13) the perturbative expansion of the $\beta$-function to all orders takes the form ${ }^{2}$

$$
\left.\beta_{I}(G)\right|_{g_{1}=-\lambda_{1}, g_{2}=-\lambda_{2}}=G_{I} f\left(\lambda_{1}^{2}+\lambda_{2}^{2}\right)
$$

\footnotetext{
${ }^{2}$ For case b) $\mathrm{SO}(2)$ invariance restricts $\left.\beta_{I}(G)\right|_{\lambda_{1}=3 g_{1}, \lambda_{2}=3 g_{2}}=G_{I} f_{1}\left(g_{1}{ }^{2}+g_{2}^{2}\right)+G_{I}^{\prime} f_{2}\left(g_{1}{ }^{2}+g_{2}^{2}\right)$ where $G_{I}^{\prime}=g_{1}\left(g_{1}^{2}-3 g_{2}^{2}\right)(1,0,-1,0)+g_{2}\left(g_{2}^{2}-3 g_{1}^{2}\right)(0,1,0,-1)$. The perturbative results to three loops give $f_{1}(x)=-\frac{1}{2} \varepsilon-\frac{35}{6} x-\frac{6241}{108} x^{2}-\left(\frac{7287775}{7776}+\frac{1061}{3} \zeta_{3}\right) x^{3}$ and $f_{2}(x)=-3-\frac{763}{18} x-\left(\frac{982045}{1296}+310 \zeta_{3}\right) x^{2}$.
} 
with

$$
f(x)=-\frac{1}{2} \varepsilon+\sum_{n \geq 1} b_{n} x^{n}, \quad b_{1}=\frac{1}{2}, \quad b_{2}=-\frac{83}{36}, \quad b_{3}=-\frac{10627}{2592}-\zeta(3) .
$$

The fixed point in this case then arises from the zeros of $f(x)$

$$
x_{*}=\varepsilon+\frac{83}{18} \varepsilon^{2}+\left(\frac{21913}{432}+2 \zeta_{3}\right) \varepsilon^{3}+\mathrm{O}\left(\varepsilon^{4}\right) .
$$

Other fixed points require complex couplings and so are not considered here.

Following from (2.15) the perturbative expansion the anomalous dimension matrix then takes the form

$$
\left.\gamma(G)\right|_{g_{1}=-\lambda_{1}, g_{2}=-\lambda_{2}}=\sum_{n \geq 1} a_{n}\left(\lambda_{1}^{2}+\lambda_{2}^{2}\right)^{n} \mathbb{1}
$$

with

$$
a_{1}=\frac{1}{6}, \quad a_{2}=-\frac{11}{108}, \quad a_{3}=\frac{5537}{7776}-\frac{1}{3} \zeta_{3} .
$$

To three loops

$$
\gamma\left(G_{*}\right)=\left(\frac{1}{6} \varepsilon+\frac{2}{3} \varepsilon^{2}+\frac{443}{54} \varepsilon^{3}+\mathrm{O}\left(\varepsilon^{4}\right)\right) \mathbb{1} .
$$

As a consequence of (2.16) at a fixed point satisfying a) in (2.13) by considering the derivative of (2.16) at $\theta=0$

$$
\left.H_{I} \partial_{I} \beta_{J}(G)\right|_{G=G_{*}}=0
$$

which gives in this case

$$
\left[H_{I}\right]=\left(\lambda_{2 *}-\lambda_{1 *}-\lambda_{2 *} \lambda_{1 *}\right) .
$$

As a consequence $\left.\operatorname{det}\left[\partial_{I} \beta_{J}(G)\right]\right|_{G=G_{*}}=0$ so that the matrix $\left.\left[\partial_{I} \beta_{J}(G)\right]\right|_{G=G_{*}}$ has a zero eigenvalue. At lowest order

$$
\begin{aligned}
{\left.\left[\partial_{I} \beta_{J}(G)^{(1)}\right]\right|_{g_{1}=-\lambda_{1}, g_{2}=-\lambda_{2}}=} & -\frac{1}{2} \varepsilon \mathbb{1} \\
& -\left(\begin{array}{cccc}
2 \lambda_{1}^{2}+\frac{9}{4} \lambda_{2}^{2} & -\frac{1}{4} \lambda_{1} \lambda_{2} & \frac{7}{6} \lambda_{1}^{2}+\frac{11}{12} \lambda_{2}^{2} & \frac{1}{4} \lambda_{1} \lambda_{2} \\
-\frac{1}{4} \lambda_{1} \lambda_{2} & \frac{9}{4} \lambda_{1}^{2}+2 \lambda_{2}^{2} & \frac{1}{4} \lambda_{1} \lambda_{2} & \frac{11}{12} \lambda_{1}^{2}+\frac{7}{6} \lambda_{2}^{2} \\
\frac{7}{2} \lambda_{1}^{2}+\frac{11}{4} \lambda_{2}^{2} & \frac{3}{4} \lambda_{1} \lambda_{2} & -\frac{1}{3} \lambda_{1}^{2}+\frac{5}{12} \lambda_{2}^{2} & -\frac{3}{4} \lambda_{1} \lambda_{2} \\
\frac{3}{4} \lambda_{1} \lambda_{2} & \frac{11}{4} \lambda_{1}^{2}+\frac{7}{2} \lambda_{2}^{2} & -\frac{3}{4} \lambda_{1} \lambda_{2} & \frac{5}{12} \lambda_{1}^{2}-\frac{1}{3} \lambda_{2}^{2}
\end{array}\right) .
\end{aligned}
$$

The eigenvectors at the fixed point are unchanged by higher order corrections to $\beta$ although the eigenvalues are modified. Scaling operators with definite scale dimension are determined by the left eigenvectors of $\left.\left[\partial_{I} \beta_{J}(G)\right]\right|_{G=G_{*}}$. There is always a zero mode given by (2.26). The other three left eigenvectors and corresponding eigenvalues to three loops are given by

$$
\begin{aligned}
& \left(\lambda_{1 *} \lambda_{2 *}-\lambda_{1 *}-\lambda_{2 *}\right), \quad \varepsilon-\frac{83}{18} \varepsilon^{2}-\left(\frac{38183}{648}+4 \zeta_{3}\right) \varepsilon^{3},
\end{aligned}
$$

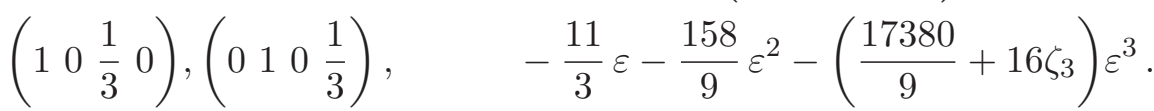

There are two degenerate relevant operators. 
An alternative reduction to that in (2.13) arises if we impose

$$
\begin{aligned}
\left(\left(\lambda_{1}+g_{1}\right)-i\left(\lambda_{2}+g_{2}\right)\right)^{3} & =C\left(\left(\lambda_{1}-3 g_{1}\right)+i\left(\lambda_{2}-3 g_{2}\right)\right), \\
\left(\left(\lambda_{1}+g_{1}\right)-i\left(\lambda_{2}+g_{2}\right)\right)^{2}\left(\left(\lambda_{1}-3 g_{1}\right)-i\left(\lambda_{2}-3 g_{2}\right)\right) & =C^{\prime}\left(\left(\lambda_{1}+g_{1}\right)+i\left(\lambda_{2}+g_{2}\right)\right),
\end{aligned}
$$

with $C, C^{\prime}$ real invariants such that $I_{2}^{3}=C^{2} I_{1}, I_{3}=C I_{1}=C^{\prime} I_{2}, I_{4}=0$. Subject to $(2.29)$

$$
\left(\begin{array}{c}
\beta_{\lambda_{1}}+\beta_{g_{1}} \\
\beta_{\lambda_{2}}+\beta_{g_{2}}
\end{array}\right)=F\left(\begin{array}{c}
\lambda_{1}+g_{1} \\
\lambda_{2}+g_{2}
\end{array}\right), \quad\left(\begin{array}{c}
\beta_{\lambda_{1}}-3 \beta_{g_{1}} \\
\beta_{\lambda_{2}}-3 \beta_{g_{2}}
\end{array}\right)=F^{\prime}\left(\begin{array}{c}
\lambda_{1}-3 g_{1} \\
\lambda_{2}-3 g_{2}
\end{array}\right),
$$

with $F, F^{\prime}$ functions of invariants. To find fixed points it is then sufficient to require just $F=F^{\prime}=0$ where $F, F^{\prime}$ depend on two independent variables. For a perturbative expansion valid away from a fixed point it is necessary that $C, C^{\prime}$ are expressible as a series expansion in the couplings. This can be achieved in (2.29) by requiring

$$
\lambda_{1} \lambda_{2}=g_{1} g_{2}, \quad \lambda_{1} g_{1}=g_{2}^{2}, \quad \lambda_{2} g_{2}=g_{1}^{2}, \quad C=C^{\prime}=I_{1}=I_{2} .
$$

In this case $F=F^{\prime}$ and

$$
\beta_{I}(G)=G_{I} F\left(I_{1}\right), \quad \gamma(G)=\left(\begin{array}{c}
\lambda_{1}+g_{1} \\
\lambda_{2}+g_{2}
\end{array}\right)\left(\lambda_{1}+g_{1} \lambda_{2}+g_{2}\right) G\left(I_{1}\right) .
$$

As (2.31) implies (2.14) this corresponds to a decoupled free field theory combined with a single field $\phi^{3}$ theory. Note that since $\operatorname{det} \gamma=0$ in this case there is always a zero anomalous dimension. To three loops,

$$
\begin{aligned}
& F(x)=-\frac{1}{2} \varepsilon-\frac{3}{4} x-\frac{125}{144} x^{2}-\left(\frac{33085}{20736}+\frac{5}{8} \zeta_{3}\right) x^{3}, \\
& G(x)=\frac{1}{12}+\frac{13}{432} x+\left(\frac{5195}{62208}-\frac{1}{24} \zeta_{3}\right) x^{2} .
\end{aligned}
$$

Of course $F(x)$ has no perturbative zeros for real couplings for $\varepsilon>0$.

\section{Two flavour scalar theory in $4-\varepsilon$ dimensions}

The structure of fixed points can be fully analysed for just two scalars [26]. A general two component $\phi^{4}$ theory is determined by the potential

$$
V(\phi)=\frac{1}{24} \lambda_{i j k l} \phi_{i} \phi_{j} \phi_{k} \phi_{l}=\frac{1}{24} \lambda_{1} \phi_{1}^{4}+\frac{1}{24} \lambda_{2} \phi_{2}^{4}+\frac{1}{4} \lambda_{0} \phi_{1}^{2} \phi_{2}^{2}+\frac{1}{6} g_{1} \phi_{1}^{3} \phi_{2}+\frac{1}{6} g_{2} \phi_{1} \phi_{2}^{3},
$$

with couplings $G_{I}=\left(\lambda_{1}, \lambda_{2}, \lambda_{0}, g_{1}, g_{2}\right)$. The transformations of the couplings $G_{I} \rightarrow G_{\theta, I}$, generating equivalent theories as in (2.8), induced by (2.6), can be decomposed into a singlet and two real two dimensional vectors. The transformations of the couplings may be reduced to irreducible components as

$$
I_{1}=\lambda_{1}+\lambda_{2}+2 \lambda_{0}, \quad v_{4}=\left(\begin{array}{c}
\frac{1}{4}\left(\lambda_{1}+\lambda_{2}-6 \lambda_{0}\right) \\
g_{1}-g_{2}
\end{array}\right), \quad v_{2}=\left(\begin{array}{c}
\frac{1}{2}\left(\lambda_{1}-\lambda_{2}\right) \\
g_{1}+g_{2}
\end{array}\right),
$$


with $v_{2}, v_{4}$ transforming as in (2.11). The invariants in this case in addition to $I_{1}$ are then

$$
\begin{aligned}
I_{2} & =\frac{1}{16}\left(\lambda_{1}+\lambda_{2}-6 \lambda_{0}\right)^{2}+\left(g_{1}-g_{2}\right)^{2}, \\
I_{3} & =\frac{1}{4}\left(\lambda_{1}-\lambda_{2}\right)^{2}+\left(g_{1}+g_{2}\right)^{2}, \\
I_{4}+i I_{5} & =\left(\frac{1}{2}\left(\lambda_{1}-\lambda_{2}\right)-i\left(g_{1}+g_{2}\right)\right)^{2}\left(\frac{1}{4}\left(\lambda_{1}+\lambda_{2}-6 \lambda_{0}\right)+i\left(g_{1}-g_{2}\right)\right),
\end{aligned}
$$

where $I_{4}^{2}+I_{5}^{2}=I_{2} I_{3}^{2}$. $I_{5}$ is odd under $\lambda_{1} \leftrightarrow \lambda_{2}, g_{1} \leftrightarrow g_{2}$.

From (3.2) invariant subspaces are also given in this case by setting two of $I_{1}, v_{2}, v_{4}$ to zero or

$$
\lambda_{1}=\lambda_{2}=-\lambda_{0}, g_{1}=-g_{2}, \quad \lambda_{1}=-\lambda_{2}, \lambda_{0}=0, g_{1}=g_{2}, \quad \lambda_{1}=\lambda_{2}=3 \lambda_{0}, g_{1}=g_{2}=0,
$$

corresponding to $\lambda_{i j k l}$ being symmetric traceless, $\lambda_{i j k l}=\delta_{(i j} s_{k l)}, s_{k k}=0, s_{11}=-s_{22}=$ $\lambda_{1}, s_{12}=2 g_{1}$, and $\lambda_{i j k l}=3 \lambda_{0} \delta_{(i j} \delta_{k l)}$. For the last case $V(\phi)=\frac{1}{8} \lambda_{0}\left(\phi_{1}^{2}+\phi_{2}^{2}\right)^{2}$ describing an $O(2)$ invariant theory. Setting $\lambda_{1}=\lambda_{2}=-\lambda_{0}, g_{1}=g_{2}=0$ gives a $\mathbb{Z}_{4}$ invariant potential. For a decoupled theory we require $g_{\theta, 1}=g_{\theta, 2}=\lambda_{\theta, 0}=0$ for some $\theta$. This gives the constraint $I_{1}^{2}=16 I_{2}, I_{1} I_{3}=4 I_{4}$ or

$$
\begin{aligned}
2 \lambda_{0}^{2}-\left(\lambda_{1}+\lambda_{2}\right) \lambda_{0}+\left(g_{1}-g_{2}\right)^{2} & =0, \\
\left(g_{1}+g_{2}\right)^{2}\left(2 \lambda_{0}-\lambda_{1}-\lambda_{2}\right)+2\left(\lambda_{1}-\lambda_{2}\right)\left(g_{1}^{2}-g_{2}^{2}\right)-\lambda_{0}\left(\lambda_{1}-\lambda_{2}\right)^{2} & =0 .
\end{aligned}
$$

For arbitrary quartic potentials $V(\phi)$ as in (3.1) the corresponding $\beta$-function can be written as in $(2.2)$, with now $\varepsilon=4-d$, where now to three loops from [27]

$$
\begin{aligned}
\bar{\beta}_{V}= & \frac{1}{2} V_{i j} V_{i j}-\frac{1}{2} V_{i j} V_{i k l} V_{j k l}+\frac{1}{4} \lambda_{i j m n} \lambda_{k l m n} V_{i k} V_{j l}-\frac{3}{16} \lambda_{i k l m} \lambda_{j k l m} V_{i n} V_{j n} \\
& +\lambda_{i j k l}\left(2 V_{i m} V_{k l n} V_{l m n}-\frac{1}{4} V_{m n} V_{i j m} V_{k l n}\right) \\
& -\frac{1}{8} V_{i k l} V_{j k l} V_{i m n} V_{j m n}+\frac{1}{2} \zeta_{3} V_{i j k} V_{i l m} V_{j l n} V_{k m n}, \\
\gamma_{i j}{ }^{(2)}= & \frac{1}{12} \lambda_{i k l m} \lambda_{j k l m}, \quad \gamma_{i j}{ }^{(3)}=-\frac{1}{16} \lambda_{i k l m} \lambda_{j k n p} \lambda_{l m n p} .
\end{aligned}
$$

The lowest order contributions to the $\beta$-functions are then easily determined, taking now $G_{I} \rightarrow(4 \pi)^{2} G_{I}$,

$$
\begin{aligned}
& \beta_{\lambda_{1}}{ }^{(1)}=-\varepsilon \lambda_{1}+3\left(\lambda_{1}^{2}+\lambda_{0}^{2}+2 g_{1}^{2}\right), \\
& \beta_{\lambda_{2}}{ }^{(1)}=-\varepsilon \lambda_{2}+3\left(\lambda_{2}^{2}+\lambda_{0}^{2}+2 g_{2}^{2}\right), \\
& \beta_{\lambda_{0}}{ }^{(1)}=-\varepsilon \lambda_{0}+\left(\lambda_{1}+\lambda_{2}\right) \lambda_{0}+4 \lambda_{0}^{2}+2 g_{1}^{2}+2 g_{2}^{2}+2 g_{1} g_{2}, \\
& \beta_{g_{1}}{ }^{(1)}=-\varepsilon g_{1}+3\left(2 \lambda_{0} g_{1}+\lambda_{0} g_{2}+\lambda_{1} g_{1}\right), \\
& \beta_{g_{2}}{ }^{(1)}=-\varepsilon g_{2}+3\left(\lambda_{0} g_{1}+2 \lambda_{0} g_{2}+\lambda_{2} g_{2}\right) .
\end{aligned}
$$

The anomalous dimension matrix is first non zero at two loops

$$
\gamma^{(2)}=\frac{1}{12}\left(\begin{array}{cc}
\lambda_{1}^{2}+3 \lambda_{0}^{2}+3 g_{1}^{2}+g_{2}^{2} & 3 \lambda_{0}\left(g_{1}+g_{2}\right)+\lambda_{1} g_{2}+\lambda_{2} g_{1} \\
3 \lambda_{0}\left(g_{1}+g_{2}\right)+\lambda_{1} g_{2}+\lambda_{2} g_{1} & \lambda_{2}^{2}+3 \lambda_{0}^{2}+g_{1}^{2}+3 g_{2}^{2}
\end{array}\right) .
$$


Finding fixed points with multiple couplings is a non trivial exercise. Analysing first the zeros for real couplings determined by the one loop $\beta$-functions imposes various constraints. These constraints reduce the representation content of the couplings to a single invariant so that higher order contributions to the $\beta$-functions are determined by a function of a single variable. There are three cases

a) $\quad \lambda_{1}=\lambda_{2}, \quad g_{1}=-g_{2}, 2 g_{1}^{2}=\lambda_{0}\left(\lambda_{1}-\lambda_{0}\right), \quad \Rightarrow \beta_{I}(G)=G_{I} f_{a}\left(\lambda_{1}+\lambda_{0}\right)$,

b) $\lambda_{0}^{2}=\lambda_{1} \lambda_{2}=g_{1} g_{2}, \quad g_{1}^{2}=\lambda_{1} \lambda_{0}, \quad g_{2}^{2}=\lambda_{2} \lambda_{0}, \quad \lambda_{0} g_{1}=\lambda_{1} g_{2}, \quad \lambda_{0} g_{2}=\lambda_{2} g_{1}$,

$$
\begin{aligned}
& \Rightarrow\left(\frac{1}{2}\left(\lambda_{1}-\lambda_{2}\right)+i\left(g_{1}+g_{2}\right)\right)^{2}=I_{1}\left(\frac{1}{4}\left(\lambda_{1}+\lambda_{2}-6 \lambda_{0}\right)+i\left(g_{1}-g_{2}\right)\right), \\
& \Rightarrow \beta_{I}(G)=G_{I} f_{b}\left(\lambda_{1}+\lambda_{2}+2 \lambda_{0}\right),
\end{aligned}
$$

c) $\quad \lambda_{1}=\lambda_{2}=3 \lambda_{0}, \quad g_{1}=g_{2}=0, \quad \Rightarrow \beta_{I}(G)=G_{I} f_{c}\left(\lambda_{0}\right)$.

In case a) $I_{2}=\frac{1}{16} I_{1}^{2}, I_{3}=I_{4}=I_{5}=0$ while in case b) $4 I_{2}=I_{3}=\frac{1}{4} I_{1}^{2}, I_{4}=\frac{1}{16} I_{1}^{3}, I_{5}=0$ and in case c) only $I_{1}$ is non zero.

From perturbative results to three loops the functions $f_{a, b, c}$ in (3.9) and their zeros as an expansion in $\varepsilon$ to $\mathrm{O}\left(\varepsilon^{3}\right)$ are given by

$$
\begin{aligned}
f_{a}(x)=f_{b}(x) & =-\varepsilon+3 x-\frac{17}{3} x^{2}+\left(\frac{145}{8}+12 \zeta_{3}\right) x^{3}, \\
x_{*} & =\frac{1}{3} \varepsilon+\frac{17}{81} \varepsilon^{2}+\left(\frac{709}{17496}-\frac{4}{27} \zeta_{3}\right) \varepsilon^{3}, \\
f_{c}(x) & =-\varepsilon+10 x-60 x^{2}+\left(617+384 \zeta_{3}\right) x^{3}, \\
x_{*} & =\frac{1}{10} \varepsilon+\frac{3}{50} \varepsilon^{2}+\left(\frac{103}{10000}-\frac{24}{625} \zeta_{3}\right) \varepsilon^{3} .
\end{aligned}
$$

The zeros determine fixed points. Case a) is equivalent to two decoupled Ising models, within the $\varepsilon$-expansion, corresponding to taking $\lambda_{0 *}=g_{1 *}=g_{2 *}=0, \lambda_{1 *}=\frac{1}{3} \varepsilon+\mathrm{O}\left(\varepsilon^{2}\right)$ and also to the theory obtained by taking $\lambda_{0 *}=\lambda_{1 *}, g_{1 *}=g_{2 *}=0, \lambda_{1 *}=\frac{1}{6} \varepsilon+\mathrm{O}\left(\varepsilon^{2}\right)$. Case b) is described by an Ising model and a free field theory, it is equivalent to taking $\lambda_{2 *}=\lambda_{0 *}=g_{1 *}=g_{2 *}=0$ when $\phi_{2}$ has no interaction. It is easy to check that these cases satisfy (3.5). Case c) is the $O(2)$ symmetric Heisenberg fixed point. Although the original theory defined by (3.1) has in general just a reflection $\mathbb{Z}_{2}$ symmetry, the fixed points all have at least a $\mathbb{Z}_{2} \times \mathbb{Z}_{2}$ reflection symmetry.

For case a) the left eigenvectors and eigenvalues of $\left.\left[\partial_{I} \beta_{J}(G)\right]\right|_{G=G_{*}}$ always include a zero mode given by

$$
\left(-4 g_{1 *}-4 g_{1 *} 4 g_{1 *} \lambda_{1 *}-3 \lambda_{0 *}-\lambda_{1 *}+3 \lambda_{0 *}\right),
$$

and to $\mathrm{O}\left(\varepsilon^{3}\right)$ the results for the others are given by

$$
\begin{aligned}
& \left(\lambda_{1 *} \lambda_{1 *} \lambda_{0 *} g_{1 *}-g_{1 *}\right),\left(\lambda_{1 *}-\lambda_{0 *} \lambda_{0 *}-\lambda_{1 *} 0 g_{1 *} g_{1 *}\right), \quad \varepsilon-\frac{17}{27} \varepsilon^{2}+\left(\frac{1603}{2916}+\frac{8}{9} \zeta_{3}\right) \varepsilon^{3}, \\
& \left(-2 \lambda_{0 *} 2 \lambda_{0 *} 0 \lambda_{1 *} \lambda_{1 *}\right), \quad \frac{1}{54} \varepsilon^{2}+\frac{109}{5832} \varepsilon^{3}, \\
& \left(\lambda_{0 *} \lambda_{0 *} \frac{1}{3}\left(\lambda_{1 *}-2 \lambda_{0 *}\right)-g_{1 *} g_{1 *}\right), \quad-\frac{1}{3} \varepsilon+\frac{19}{81} \varepsilon^{2}+\left(\frac{937}{8748}-\frac{8}{27} \zeta_{3}\right) \varepsilon^{3} .
\end{aligned}
$$


The eigenvectors lie within the invariant subspaces in (3.4). There is one slightly relevant operator. For case b) the corresponding results are

$$
\begin{aligned}
& \left(-4 g_{1 *} 4 g_{2 *} 2\left(g_{1 *}-g_{2 *}\right) \lambda_{1 *}-3 \lambda_{0 *}-\lambda_{2 *}+3 \lambda_{0 *}\right), \quad 0 \\
& \left(-4 g_{2 *} 4 g_{1 *}-2\left(g_{1 *}-g_{2 *}\right)-\lambda_{2 *}+3 \lambda_{0 *} \lambda_{1 *}-3 \lambda_{0 *}\right), \quad-\varepsilon+\frac{1}{108} \varepsilon^{2}+\frac{109}{11664} \varepsilon^{3} \text {, } \\
& \left(\lambda_{1 *} \lambda_{2 *} \lambda_{0 *} g_{1 *} g_{2 *}\right), \quad \varepsilon-\frac{17}{27} \varepsilon^{2}+\left(\frac{1603}{2916}+\frac{8}{9} \zeta_{3}\right) \varepsilon^{3}, \\
& \left(\lambda_{2 *} \lambda_{1 *} \lambda_{0 *}-g_{2 *}-g_{1 *}\right), \quad-\varepsilon, \\
& \left(6 \lambda_{0 *} 6 \lambda_{0 *} \lambda_{1 *}+\lambda_{2 *}-4 \lambda_{0 *}-3\left(g_{1 *}-g_{2 *}\right) 3\left(g_{1 *}-g_{2 *}\right)\right) \text {, } \\
& -\frac{2}{3} \varepsilon+\frac{19}{162} \varepsilon^{2}+\left(\frac{937}{17496}-\frac{4}{27} \zeta_{3}\right) \varepsilon^{3} \text {. }
\end{aligned}
$$

For the $O(2)$ symmetric case c) the left eigenvectors and associated eigenvalues are

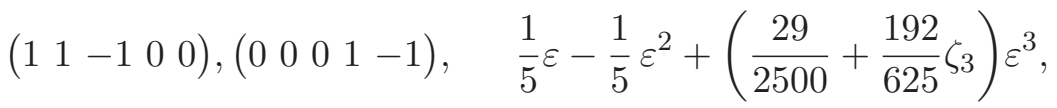

$$
\begin{aligned}
& \left(\begin{array}{lllll}
1 & -1 & 0 & 0 & 0
\end{array}\right),\left(\begin{array}{lllll}
0 & 0 & 0 & 1 & 1
\end{array}\right), \quad \frac{4}{5} \varepsilon-\frac{1}{2} \varepsilon^{2}+\left(\frac{1777}{5000}+\frac{408}{625} \zeta_{3}\right) \varepsilon^{3},
\end{aligned}
$$

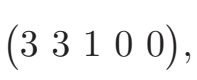

$$
\begin{aligned}
& \varepsilon-\frac{3}{5} \varepsilon^{2}+\left(\frac{257}{500}+\frac{96}{125} \zeta_{3}\right) \varepsilon^{3} \text {. }
\end{aligned}
$$

All perturbations then correspond to irrelevant operators.

Assuming the constraints on the couplings in (3.9) we have to all orders

$$
\begin{aligned}
\left.\gamma\right|_{\lambda_{1}=\lambda_{2}, g_{1}=-g_{2}, 2 g_{1}{ }^{2}=\lambda_{0}\left(\lambda_{1}-\lambda_{0}\right)} & =g_{a}\left(\lambda_{1}+\lambda_{0}\right) \mathbb{1}, \\
\left.\gamma\right|_{\lambda^{2}=\lambda_{1} \lambda_{2}=g_{1} g_{2}, g_{1}{ }^{2}=\lambda_{1} \lambda_{0}, g_{2}{ }^{2}=\lambda_{2} \lambda_{0}, \lambda_{0} g_{1}=\lambda_{1} g_{2}, \lambda_{0} g_{2}=\lambda_{2} g_{1}} & =g_{b}\left(\lambda_{1}+\lambda_{2}+2 \lambda_{0}\right) \\
\left.\gamma\right|_{\lambda_{1}=\lambda_{2}=3 \lambda_{0}, g_{1}=g_{2}=0} & =g_{c}\left(\lambda_{0}\right) \mathbb{1}
\end{aligned}
$$

with

$$
x g_{b}(x)=g_{a}(x) .
$$

To three loops

$$
g_{a}(x)=\frac{1}{12} x^{2}-\frac{1}{16} x^{3}, \quad g_{c}(x)=x^{2}-\frac{5}{2} x^{3}
$$

It is easy to see, subject to the necessary conditions for case b), that $\operatorname{det}\left(\begin{array}{cc}\lambda_{1}+\lambda_{0} & g_{1}+g_{2} \\ g_{1}+g_{2} & \lambda_{2}+\lambda_{0}\end{array}\right)=0$ so that the eigenvalues are $\lambda_{1}+\lambda_{2}+2 \lambda_{0}, 0$. The non zero anomalous dimensions at the fixed points to three loops are then

$$
\frac{1}{108}\left(\varepsilon^{2}+\frac{109}{108} \varepsilon^{3}\right), \quad \frac{1}{100}\left(\varepsilon^{2}+\frac{19}{20} \varepsilon^{3}\right),
$$

for the Ising model case and the $O(2)$ theory respectively.

The constraints in (3.9) reduce the RG flow to one dimensional trajectories up to an $O(2)$ equivalence. Relaxing the restrictions such as imposing just $v_{2}=0$ the RG flow is 
restricted to a two dimensional surface since

$$
\begin{aligned}
\beta_{I}(G) & =G_{I} a\left(\lambda_{1}+\lambda_{0}, J\right)+G_{I}^{0} b\left(\lambda_{1}+\lambda_{0}, J\right), \quad \gamma(G)=c\left(\lambda_{1}+\lambda_{0}, J\right) \mathbb{1}, \\
G_{I} & =\left(\lambda_{1}, \lambda_{1}, \lambda_{0}, g_{1},-g_{1}\right), \quad G_{I}^{0}=(3,3,1,0,0), \quad J=\lambda_{0}\left(\lambda_{1}-\lambda_{0}\right)-2 g_{1}^{2} .
\end{aligned}
$$

In terms of $(3.9) f_{a}(x)=a(x, 0), f_{c}(x)=a\left(4 x, 2 x^{2}\right)+2 x b\left(4 x, 2 x^{2}\right)$. From perturbation theory to three loops

$$
\begin{aligned}
& a(x, y)=-\varepsilon+3 x-\frac{1}{3}\left(17 x^{2}+2 y\right)+\frac{1}{8} x\left(\left(145+96 \zeta_{3}\right) x^{2}-168 y\right) \\
& b(x, y)=-y+4 x y-\frac{1}{8}\left(\left(89+96 \zeta_{3}\right) x^{2}+38 y\right) y \\
& c(x, y)=\frac{1}{12}\left(x^{2}-2 y\right)-\frac{1}{16} x\left(x^{2}-3 y\right) .
\end{aligned}
$$

Fixed points require $a(x, y)=b(x, y)=0$ unless $G_{I} \propto G_{I}^{0}$ requiring $\lambda_{1}=3 \lambda_{0}, g_{1}=0$ so that $\lambda_{1}+\lambda_{0}=4 \lambda_{0}, J=2 \lambda_{0}^{2}$ and $c\left(4 x, 2 x^{2}\right)=x^{2}-\frac{5}{2} x^{3}$ corresponding to the $O(2)$ invariant theory. Perturbatively $b(x, y)=0$ requires $y=0$. On this surface the RG flow links the fixed points corresponding to case a) and case c) in (3.9).

\section{Scalar theories with reduced symetry in $6-\varepsilon$ dimensions}

For $\mathcal{N}$ scalars $\phi_{i}$ the kinetic term has $O(\mathcal{N})$ symmetry. However for a renormalisable theory in $6-\varepsilon$ dimensions with a cubic potential there is of course no $O(\mathcal{N})$ invariant theory. For $\mathcal{N}=N+1$ and decomposing $\phi_{i}=\left(\sigma, \varphi_{i}\right)$ there is an $O(N)$ invariant theory with $\sigma=\phi_{0}$ a singlet and $\varphi_{i}$ transforming as a vector. As analysed in detail in [23] there is a fixed point with $O(N)$ symmetry in $6-\varepsilon$ dimensions. Here we consider perturbations which break the $O(N)$ symmetry but maintain the reflection symmetry under $\varphi_{i} \rightarrow-\varphi_{i}$ based on the potential with three couplings

$$
V(\phi)=\frac{1}{6} \lambda_{i j k} \phi_{i} \phi_{j} \phi_{k}=\frac{1}{2} g \sigma \varphi_{i} \varphi_{i}+\frac{1}{2} h \sigma d_{i j} \varphi_{i} \varphi_{j}+\frac{1}{6} \lambda \sigma^{3},
$$

where $d_{i j}=d_{j i}$ is assumed to satisfy, for a convenient choice of normalisation,

$$
d_{i i}=0, \quad d_{i k} d_{k j}=\delta_{i j}+b d_{i j} .
$$

For $h=0$ this is just the $O(N)$ invariant theory. Theories in which the tensors $d_{i j}$ are related by $O(N)$ transformations are equivalent. Invariance under reflections $\sigma \rightarrow-\sigma$ ensures that $(\lambda, g, h) \simeq(-\lambda,-g,-h)$ defining equivalent theories.

To lowest order the $\beta$-function in general is given by, after rescaling $\lambda_{i j k} \rightarrow(4 \pi)^{\frac{3}{2}} \lambda_{i j k}$,

$$
4 \beta_{i j k}=\frac{\partial}{\partial \lambda_{i j k}} A, \quad A=-\varepsilon \lambda_{i j k} \lambda_{i j k}-\lambda_{i j k} \lambda_{i l m} \lambda_{j l n} \lambda_{k m n}+\frac{1}{4} \lambda_{i k l} \lambda_{j k l} \lambda_{i m n} \lambda_{j m n} .
$$

Under RG flow to the IR $A$ decreases. However since the lowest order result for $A$ in (4.3) has no global minimum, the couplings may become large under RG flow and the approximation loses its validity. At any local minimum of $A$ at which $\beta_{i j k}=0$ with $\lambda_{* i j k}=\mathrm{O}(\varepsilon)[16]$

$$
A_{*}=-\frac{1}{2} \varepsilon \lambda_{* i j k} \lambda_{* i j k}=-6 \varepsilon \operatorname{tr}\left(\gamma_{*}\right),
$$


with $\gamma_{*}$ the lowest order contribution to the anomalous dimension at the critical point.

For the three coupling theory in (4.1) the general form (2.3) determines

$$
\begin{aligned}
& \beta_{\lambda}=-\frac{1}{2} \varepsilon \lambda-\frac{3}{4} \lambda^{3}-N\left(g^{3}-\frac{1}{4}\left(g^{2}+h^{2}\right) \lambda+3 g h^{2}+b h^{3}\right), \\
& \beta_{g}=-\frac{1}{2} \varepsilon g+\frac{1}{12}(N-8) g^{3}-\left(g^{2}+h^{2}\right) \lambda+\frac{1}{12} \lambda^{2} g+\frac{1}{12}(N-24) g h^{2}-\frac{2}{3} b h^{3}, \\
& \beta_{h}=-\frac{1}{2} \varepsilon h+\frac{1}{12}(N-24) g^{2} h-2 g \lambda h+\frac{1}{12} \lambda^{2} h-2 b g h^{2}-b \lambda h^{2}+\left(\frac{1}{12}(N-8)-\frac{2}{3} b^{2}\right) h^{3},
\end{aligned}
$$

and also the anomalous dimensions for $\sigma, \varphi$ become

$$
\begin{aligned}
\gamma_{\sigma} & =\frac{1}{12}\left(N\left(g^{2}+h^{2}\right)+\lambda^{2}\right), \\
\gamma_{\varphi i j} & =\frac{1}{6}\left(g^{2}+h^{2}\right) \delta_{i j}+\frac{1}{6}\left(2 g h+b h^{2}\right) d_{i j} .
\end{aligned}
$$

Of course for $h=0$ these reduce to standard results for the $O(N)$ symmetric theory.

For $h=0$ the fixed points for were analysed by Fei et al. [23]. In the $\varepsilon$ expansion possible fixed points are in general determined by the lowest order one loop contributions to the $\beta$-functions, higher orders give an expansion in powers of $\varepsilon$ for the positions of the fixed points. At large $N$ there are three inequivalent $O(N)$ fixed points, obtained by the vanishing of $\beta_{\lambda}, \beta_{h}$ in (4.5), with non zero real $\left(\lambda_{*}, g_{*}\right) \simeq\left(-\lambda_{*},-g_{*}\right)$, given by, to first order in $\varepsilon$,

$$
g_{*}^{2} \approx \frac{6}{N} \varepsilon, \quad \lambda_{*}^{2} \approx \frac{6^{3}}{N} \varepsilon, \quad g_{*} \lambda_{*}>0, \quad \text { and } \quad g_{*}^{2} \approx \frac{5}{N} \varepsilon, \quad \lambda_{*}^{2} \approx \varepsilon
$$

Defining $x=\lambda / g$ and then writing the one loop $\beta$-functions as

$$
\begin{array}{ll}
\beta_{\lambda}=-\frac{1}{2} \varepsilon \lambda+g^{3} f_{\lambda}(x), & f_{\lambda}(x)=-\frac{1}{4}\left(3 x^{3}+4 N-N x\right), \\
\beta_{g}=-\frac{1}{2} \varepsilon g+g^{3} f_{g}(x), & f_{g}(x)=\frac{1}{12}\left(N-8-12 x+x^{2}\right),
\end{array}
$$

the fixed point equations require

$$
f(x)=x f_{g}(x)-f_{\lambda}(x)=\frac{1}{6}\left(5 x^{3}-6 x^{2}-(N+4) x+6 N\right)=0 .
$$

For $N>0$ this cubic equation has coincident roots, so that $f(x)=f^{\prime}(x)=0$, at

$$
\left(x_{\text {crit }}, N_{\text {crit }}\right)=(8.7453,1038.27), \quad\left(x_{\text {crit }}^{\prime}, N_{\text {crit }}^{\prime}\right)=(1.10339,1.02145) .
$$

For $N_{\text {crit }}^{\prime}<N<N_{\text {crit }}$ there is only one real solution of $f(x)=0$ with $x<0$ for any $N>0$. For $N>N_{\text {crit }}$ there are two roots for $x>0$, which merge at $x=x_{\text {crit }}$ when $\left(\lambda^{2}, N g^{2}\right)=(0.4581,6.218) \varepsilon$, and one root with $x<0$. For real couplings it is necessary that at the fixed point $f\left(x_{*}\right)=0$ then $f_{g}\left(x_{*}\right)>0$ or $\left(x_{*}-6\right)^{2}>44-N$. For large $N$ the roots are $6, \pm \sqrt{\frac{N}{5}}$ which correspond to the three inequivalent fixed points given by (4.7). For $N=1 f(x)$ has roots at $x= \pm 1, \frac{6}{5}$ but there is just one inequivalent fixed point with 
real couplings when $\lambda_{*}=-g_{*}$ and $\lambda_{*}{ }^{2}=\varepsilon$. The roots at $x=1, \frac{6}{5}$ correspond to the fixed points which merge when $N \rightarrow N_{\text {crit }}^{\prime}$ and give $g^{2}<0$, and so both $\lambda, g$ are imaginary. In contrast to four dimensions, as a consequence of the reflection symmetry $(\lambda, g) \sim-(\lambda, g)$, the stable fixed point is not unique.

A discussion of the $\varepsilon, \varepsilon^{2}$ corrections to the bifurcation point in (4.10) is given in appendix A.

The stability matrix in this case is

$$
M=\left.\left(\begin{array}{ll}
\partial_{\lambda} \beta_{\lambda} & \partial_{g} \beta_{\lambda} \\
\partial_{\lambda} \beta_{g} & \partial_{g} \beta_{g}
\end{array}\right)\right|_{\lambda=\lambda_{*}, g=g_{*}, h=0},
$$

and, with the $\beta$-functions given by (4.5) for $h=0$.

$$
\operatorname{det} M=-\varepsilon g_{*}^{2} f^{\prime}\left(x_{*}\right), \quad \operatorname{tr} M=\varepsilon-g_{*}^{2} f^{\prime}\left(x_{*}\right), \quad x_{*}=\lambda_{*} / g_{*} \cdot f\left(x_{*}\right)=0,
$$

This determines the eigenvalues of $M$ to lowest order in $\varepsilon$

$$
\kappa_{0}=\varepsilon, \quad \kappa_{1}=-\lambda_{*} f^{\prime}\left(x_{*}\right) .
$$

For $\left(\lambda_{*}, g_{*}\right)$ to be a stable fixed point the eigenvalues must be positive which requires $f^{\prime}\left(x_{*}\right)<0$. At the roots for large $N f^{\prime}(x *) \approx-\frac{1}{6} N, \frac{1}{3} N$. When $f^{\prime}\left(x_{*}\right)=0$ there is a marginal operator. For any $x_{*}$ the corresponding $g_{*}$ is determined to this order by $g_{*}^{2}=\frac{1}{2} \varepsilon / f_{g}\left(x_{*}\right)$.

From (4.3) for $h=0$

$$
A=-\varepsilon\left(3 N g^{2}+\lambda^{2}\right)-2 N g^{3}(g+2 \lambda)-\lambda^{4}+\frac{1}{4}\left(N g^{2}+\lambda^{2}\right)^{2},
$$

which satisfies

$$
\frac{\partial}{\partial g} A=12 N \beta_{g}, \quad \frac{\partial}{\partial \lambda} A=4 \beta_{\lambda}
$$

and at a fixed point

$$
A_{*}=-\frac{1}{2}\left(3 N g_{*}^{2}+\lambda_{*}^{2}\right) \varepsilon,
$$

in accord with (4.4). This gives

$$
A_{*}=-\left.3 \frac{3 N+x^{2}}{N-8-12 x+x^{2}} \varepsilon^{2}\right|_{f(x)=0},
$$

and we may then obtain

$$
A_{*} \geq A_{* \min }=-3 \frac{3 N_{\text {crit }}+x_{\text {crit }}^{2}}{N_{\text {crit }}-8-12 x_{\text {crit }}+x_{\text {crit }}^{2}} \varepsilon^{2} \approx-9.557 \varepsilon^{2} .
$$

The minimum is attained when the fixed points merge. At the large $N$ fixed points in (4.7)

$$
A_{*} \approx-9 \varepsilon^{2},-8 \varepsilon^{2}
$$

with $A_{*}$ lower at the stable fixed point. 
The $h=0$ and $N=1$ results here are related to the discussion in section 4 by taking $\lambda_{1}=\lambda, g_{1}=g$ and $\lambda_{2}=g_{2}=0$. The fixed point obtained with $\lambda_{*}=-g_{*}$ has a $\mathbb{Z}_{3}$ symmetry as described there. For $\lambda=g$ the condition (2.14) is satisfied and the theory becomes the sum of two decoupled $N=0$ theories, with $\lambda_{1}=\lambda_{2}=\lambda / \sqrt{2}, g_{1}=g_{2}=0$.

However from (4.5) the large $N$ fixed point is unstable for non zero $h$ since

$$
\left.\partial_{h} \beta_{h}\right|_{g=g_{*}, \lambda=\lambda_{*}, h=0}=-\frac{4}{3} g_{*}^{2}-g_{*} \lambda_{*}<0 \quad \text { if } \quad N>N_{\text {crit }},
$$

so that the coupling $h$ corresponds to a relevant operator. To find fixed points which are reached for non zero $h$ we consider the linear transformation

$$
g=\frac{1}{1+\alpha^{2}}\left(g^{\prime}+\alpha^{2} h^{\prime}\right), \quad h=\frac{\alpha}{1+\alpha^{2}}\left(g^{\prime}-h^{\prime}\right), \quad \alpha^{2}-1=\alpha b .
$$

and the $\beta$-functions become

$$
\begin{aligned}
& \beta_{\lambda}=-\frac{1}{2} \varepsilon \lambda-\frac{3}{4} \lambda^{3}-m\left(g^{\prime 3}-\frac{1}{4} \lambda g^{\prime 2}\right)-n\left(h^{\prime 3}-\frac{1}{4} \lambda h^{\prime 2}\right), \\
& \beta_{g^{\prime}}=-\frac{1}{2} \varepsilon g^{\prime}+\frac{1}{12}(m-8) g^{\prime 3}-\lambda g^{\prime 2}+\frac{1}{12}\left(n h^{\prime 2}+\lambda^{2}\right) g^{\prime} \\
& \beta_{h^{\prime}}=-\frac{1}{2} \varepsilon h^{\prime}+\frac{1}{12}\left(m g^{\prime 2}+\lambda^{2}\right) h^{\prime}-\lambda h^{\prime 2}+\frac{1}{12}(n-8) h^{\prime 3}
\end{aligned}
$$

for

$$
m=\frac{1}{1+\alpha^{2}} N, \quad n=\frac{\alpha^{2}}{1+\alpha^{2}} N .
$$

For $h^{\prime}=0$ the $\beta$-functions are just those of the unperturbed $O(m)$ theory, for $m$ an integer, and so have the same fixed points, while $n$ fields become free. So long as $m g^{\prime 2}+\lambda^{2}>\varepsilon$ the $h^{\prime}$ coupling is irrelevant so under RG flow the couplings are attracted to the sub manifold corresponding to $h^{\prime}=0$. A similar reduction arises for $g^{\prime}=0$ taking $m \leftrightarrow n$. There are also fixed points for $g^{\prime}, h^{\prime}$ non zero. For $x=g^{\prime} / \lambda, y=h^{\prime} / \lambda$ the vanishing of the lowest order $\beta$-functions in (4.22) requires

$$
m\left(x^{3}-\frac{1}{6} x^{2}\right)+n\left(y^{3}-\frac{1}{6} y^{2}\right)=\frac{2}{3} x^{2}+x-\frac{5}{6}=\frac{2}{3} y^{2}+y-\frac{5}{6},
$$

with then, for any solution of (4.24), $\lambda$ at the fixed point is given by

$$
-\frac{1}{2} \varepsilon=\lambda_{*}^{2}\left(m\left(x^{3}-\frac{1}{4} x^{2}\right)+n\left(y^{3}-\frac{1}{4} y^{2}\right)+\frac{3}{4}\right) .
$$

Consistency of (4.24) requires $x=y$ or $x+y=-\frac{3}{2}$. In the first case then from (4.21) $h=0$ and $g^{\prime}=h^{\prime}=g$ and the theory reduces to the unbroken $O(N)$ theory and has the same fixed points as in (4.7) for large $N$. For the second case if $m \gg n$ then we may take $y \approx-\frac{3}{2}$ and $m x^{2} \approx 5\left(1-\frac{9}{2} n\right)$. With $n$ taking integer values this does not lead to real solutions. The two cases coincide for $x=y=-\frac{3}{4}$ which requires $N=\frac{2}{9}$.

To determine the possible values of $\alpha$ which are given in terms of $b$ in (4.21) it is necessary to find the various solutions of (4.2). To this end we consider eigenvectors 
satisfying $d_{i j} v_{j}=\lambda v_{i}$ where there are two possible eigenvalues $\lambda_{ \pm}=\frac{1}{2}\left(b \pm \sqrt{b^{2}+4}\right)$. Assuming $r \lambda_{+}$and $N-r \lambda_{-}$the trace condition gives

$$
r(N-r) b^{2}=(N-2 r)^{2}, \quad r=1, \ldots, N-1,
$$

giving, for $b=(N-2 r) / \sqrt{r(N-r)}$,

$$
\lambda_{+}=\sqrt{\frac{N-r}{r}}, \quad \lambda_{-}=-\sqrt{\frac{r}{N-r}} .
$$

Other solutions are obtained for $r \rightarrow N-r$. The solutions for $\alpha$ are then

$$
\alpha=\lambda_{ \pm} \quad \Rightarrow \quad m=r, N-r .
$$

The relevant perturbations corresponding to the coupling $h$ then break the symmetry $O(N) \rightarrow O(m) \times O(n)$ with $m+n=N$, depending on the solutions chosen for (4.2). The RG flow leads to a non trivial CFT with $O(m)$ or $O(n)$ symmetry, assuming $m$ or $n$ are large enough, while the remaining fields become free. Assuming (4.6) and (4.21) then

$$
\begin{aligned}
\gamma_{\sigma} & =\frac{1}{12}\left(m g^{\prime 2}+n h^{\prime 2}+\lambda^{2}\right), \\
\gamma_{\varphi i j} & =\frac{1}{6}\left(g^{\prime 2} \delta_{+i j}+h^{\prime 2} \delta_{-i j}\right),
\end{aligned}
$$

for $\delta_{ \pm}$projection operators given by

$$
\delta_{+i j}=\frac{1}{1+\alpha^{2}}\left(\delta_{i j}+\alpha d_{i j}\right), \quad \delta_{-i j}=\frac{1}{1+\alpha^{2}}\left(\alpha^{2} \delta_{i j}-\alpha d_{i j}\right) .
$$

The eigenvalues of $\gamma_{\varphi}$ ij are $\frac{1}{6} g^{\prime 2}, \frac{1}{6} h^{\prime 2}$ according to whether the eigenvalue of $d_{i j}$ is $\alpha,-1 / \alpha$.

The results for the $O(m) \times O(n)$ theory can be extended to higher loops. Thus the two loop $\beta$-functions are given by applying (2.4)

$$
\begin{aligned}
& \beta_{\lambda^{2}}^{(2)}=-\frac{1}{144}( 125 \lambda^{5}+m g^{\prime 2}\left(24 g^{\prime 3}+322 \lambda g^{\prime 2}+60 g^{\prime} \lambda^{2}-31 \lambda^{3}\right) \\
&+\left.n h^{\prime 2}\left(24 h^{\prime 3}+322 \lambda h^{\prime 2}+60 h^{\prime} \lambda^{2}-31 \lambda^{3}\right)\right), \\
& \beta_{g^{\prime}}^{(2)}=-\frac{1}{432} g^{\prime}\left((86 m+536) g^{\prime 4}-12(11 m-30) g^{\prime 3} \lambda+(11 m+628) g^{\prime 2} \lambda^{2}+24 g^{\prime} \lambda^{3}\right. \\
&\left.-13 \lambda^{4}-n h^{\prime 2}\left(2 h^{\prime 2}+48 h^{\prime} \lambda-11 \lambda^{2}+20 g^{\prime 2}-108 g^{\prime} h^{\prime}+84 g^{\prime} \lambda\right)\right), \\
& \beta_{h^{\prime}}^{(2)}=-\frac{1}{432} h^{\prime}\left((86 n+536) h^{\prime 4}-12(11 n-30) h^{\prime 3} \lambda+(11 n+628) h^{\prime 2} \lambda^{2}+24 h^{\prime} \lambda^{3}-13 \lambda^{4}\right. \\
&\left.\quad-m g^{\prime 2}\left(2 g^{\prime 2}+48 g^{\prime} \lambda-11 \lambda^{2}+20 h^{\prime 2}-108 g^{\prime} h^{\prime}+84 h^{\prime} \lambda\right)\right),
\end{aligned}
$$

and for the two loop anomalous dimensions

$$
\begin{gathered}
\gamma_{\sigma}^{(2)}=\frac{1}{432}\left(m g^{\prime 2}\left(2 g^{\prime 2}-11 \lambda^{2}+48 \lambda g^{\prime}\right)+n h^{\prime 2}\left(2 h^{\prime 2}-11 \lambda^{2}+48 \lambda h^{\prime}\right)+13 \lambda^{4}\right) \\
\gamma_{\varphi i j}{ }^{(2)}=-\frac{1}{432}\left(g^{\prime 2}\left((11 m-26) g^{\prime 2}+11 n h^{\prime 2}-48 g^{\prime} \lambda+11 \lambda^{2}\right) \delta_{+i j}\right. \\
\left.\quad+h^{\prime 2}\left(11 m g^{\prime 2}+(11 n-26) h^{\prime 2}-48 h^{\prime} \lambda+11 \lambda^{2}\right) \delta_{-i j}\right)
\end{gathered}
$$




\section{1 $O(m) \times O(n)$ theories in $6-\varepsilon$ dimensions}

By letting $\phi_{i}=\left(\sigma, \Phi_{a r}, \rho_{a b}\right), a, b=1, \ldots m, r=1, \ldots n$ and where $\rho_{a b}=\rho_{b a}, \rho_{a a}=0$ are $\frac{1}{2}(m-1)(m+2)$ additional scalars, the $O(N)$ symmetric theory can be broken to $O(m) \times O(n) / \mathbb{Z}_{2}, N=m n$, by extending the potential to

$$
V=\frac{1}{2} g_{\sigma} \sigma \Phi^{2}+\frac{1}{2} g_{\rho} \rho_{a b} \Phi_{a r} \Phi_{b r}+\frac{1}{6} \lambda_{\sigma} \sigma^{3}+\frac{1}{6} \lambda_{\rho} \operatorname{tr}\left(\rho^{3}\right)+\frac{1}{2} \tilde{\lambda} \sigma \operatorname{tr}\left(\rho^{2}\right) .
$$

In general there are then five couplings. For $m=2 \operatorname{tr}\left(\rho^{3}\right)=0$ so that the $\lambda_{\rho}$ coupling is irrelevant. By considering reflections of $\sigma$ and $\rho$ it is clear that there are equivalence relations

$$
\left(g_{\sigma}, g_{\rho}, \lambda_{\sigma}, \lambda_{\rho}, \tilde{\lambda}\right) \simeq\left(-g_{\sigma}, g_{\rho},-\lambda_{\sigma}, \lambda_{\rho},-\tilde{\lambda}\right) \simeq\left(g_{\sigma},-g_{\rho}, \lambda_{\sigma},-\lambda_{\rho}, \tilde{\lambda}\right) .
$$

The restricted theory with $g_{\sigma}, \lambda_{\sigma}$ and $\tilde{\lambda}$ set to zero has been discussed in [28] and the general theory in [29]. For this case there is a tractable large $n$ limit valid for arbitrary $d$ which links perturbative results in six and four dimensions.

The potential (4.33) defines a renormalisable theory in $d=6-\varepsilon$ dimensions and the $\beta$-functions have the form

$$
\begin{array}{ll}
\beta_{g_{\sigma}}=\bar{\beta}_{g_{\sigma}}+\left(-\frac{1}{2} \varepsilon+\gamma_{\sigma}+2 \gamma_{\Phi}\right) g_{\sigma}, & \beta_{g_{\rho}}=\bar{\beta}_{g_{\rho}}+\left(-\frac{1}{2} \varepsilon+\gamma_{\rho}+2 \gamma_{\Phi}\right) g_{\rho}, \\
\beta_{\lambda_{\sigma}}=\bar{\beta}_{\lambda_{\sigma}}+\left(-\frac{1}{2} \varepsilon+3 \gamma_{\sigma}\right) \lambda_{\sigma}, & \beta_{\lambda_{\rho}}=\bar{\beta}_{\lambda_{\rho}}+\left(-\frac{1}{2} \varepsilon+3 \gamma_{\rho}\right) \lambda_{\rho} \\
\beta_{\tilde{\lambda}}=\bar{\beta}_{\tilde{\lambda}}+\left(-\frac{1}{2} \varepsilon+\gamma_{\sigma}+2 \gamma_{\rho}\right) \tilde{\lambda}, &
\end{array}
$$

As a consequence of (4.34) the $\beta$-functions satisfy the identities

$$
\begin{aligned}
\beta_{g_{\sigma}, \lambda_{\sigma}, \tilde{\lambda}}\left(g_{\sigma}, g_{\rho}, \lambda_{\sigma}, \lambda_{\rho}, \tilde{\lambda}\right) & =-\beta_{g_{\sigma}, \lambda_{\sigma}, \tilde{\lambda}}\left(-g_{\sigma}, g_{\rho},-\lambda_{\sigma}, \lambda_{\rho},-\tilde{\lambda}\right), \\
\beta_{g_{\rho}, \lambda_{\rho}}\left(g_{\sigma}, g_{\rho}, \lambda_{\sigma}, \lambda_{\rho}, \tilde{\lambda}\right) & =\beta_{g_{\rho}, \lambda_{\rho}}\left(-g_{\sigma}, g_{\rho},-\lambda_{\sigma}, \lambda_{\rho},-\tilde{\lambda}\right) \\
\beta_{g_{\sigma}, \lambda_{\sigma}, \tilde{\lambda}}\left(g_{\sigma}, g_{\rho}, \lambda_{\sigma}, \lambda_{\rho}, \tilde{\lambda}\right) & =\beta_{g_{\sigma}, \lambda_{\sigma}, \tilde{\lambda}}\left(g_{\sigma},-g_{\rho}, \lambda_{\sigma},-\lambda_{\rho}, \tilde{\lambda}\right) \\
\beta_{g_{\rho}, \lambda_{\rho}}\left(g_{\sigma}, g_{\rho}, \lambda_{\sigma}, \lambda_{\rho}, \tilde{\lambda}\right) & =-\beta_{g_{\rho}, \lambda_{\rho}}\left(g_{\sigma},-g_{\rho}, \lambda_{\sigma},-\lambda_{\rho}, \tilde{\lambda}\right) .
\end{aligned}
$$

At one loop, using $\partial_{i}=\left(\partial_{\sigma}, \partial_{\Phi, a r}, \partial_{\rho, a b}\right)$, where $\partial_{\rho, a b} \rho_{c d}=\frac{1}{2}\left(\delta_{a c} \delta_{b d}+\delta_{a d} \delta_{b c}\right)-\frac{1}{m} \delta_{a b} \delta_{c d}$ and $\partial_{i} \partial_{i}=\partial_{\sigma}^{2}+\partial_{\Phi, a r} \partial_{\Phi, a r}+\partial_{\rho, a b} \partial_{\rho, a b}$ then from (2.2)

$$
\begin{aligned}
& \bar{\beta}_{g_{\sigma}}{ }^{(1)}=-g_{\sigma}{ }^{3}-g_{\sigma}{ }^{2} \lambda_{\sigma}-\frac{1}{2 m}(m-1)(m+2)\left(g_{\sigma}+\tilde{\lambda}\right) g_{\rho}^{2} \\
& \bar{\beta}_{g_{\rho}}{ }^{(1)}=-g_{\sigma}{ }^{2} g_{\rho}-2 g_{\sigma} g_{\rho} \tilde{\lambda}-\frac{1}{2 m}(m-2) g_{\rho}^{3}-\frac{1}{4 m}(m-2)(m+4) g_{\rho}^{2} \lambda_{\rho} \\
& \bar{\beta}_{\lambda_{\sigma}}{ }^{(1)}=-N g_{\sigma}{ }^{3}-\lambda_{\sigma}^{3}-\frac{1}{2}(m-1)(m+2) \tilde{\lambda}^{3}, \\
& \bar{\beta}_{\lambda_{\rho}}{ }^{(1)}=-n g_{\rho}^{3}-\frac{1}{8 m}\left(m^{2}+4 m-24\right) \lambda_{\rho}^{3}-3 \lambda_{\rho} \tilde{\lambda}^{2}, \\
& \bar{\beta}_{\tilde{\lambda}^{(1)}}{ }^{2}=-n g_{\sigma} g_{\rho}^{2}-\lambda_{\sigma} \tilde{\lambda}^{2}-\tilde{\lambda}^{3}-\frac{1}{4 m}(m-2)(m+4) \lambda_{\rho}^{2} \tilde{\lambda},
\end{aligned}
$$


with anomalous dimensions

$$
\begin{aligned}
& \gamma_{\Phi}^{(1)}=\frac{1}{12}\left(2 g_{\sigma}^{2}+\frac{1}{m}(m-1)(m+2) g_{\rho}^{2}\right), \\
& \gamma_{\sigma}^{(1)}=\frac{1}{12}\left(N g_{\sigma}^{2}+\lambda_{\sigma}^{2}+\frac{1}{2}(m-1)(m+2) \tilde{\lambda}^{2}\right), \\
& \gamma_{\rho}^{(1)}=\frac{1}{12}\left(n g_{\rho}^{2}+\frac{1}{4 m}(m-2)(m+4) \lambda_{\rho}^{2}+2 \tilde{\lambda}^{2}\right) .
\end{aligned}
$$

At two loops the results for the $\beta$-functions are rather non trivial but can be obtained from $(2.2)$

$$
\begin{aligned}
& \bar{\beta}_{g_{\sigma}}{ }^{(2)}=-\frac{1}{72}(11 N+98) g_{\sigma}{ }^{5}+\frac{1}{36}(7 N-38) g_{\sigma}{ }^{4} \lambda_{\sigma}-\frac{101}{72} g_{\sigma}{ }^{3} \lambda_{\sigma}{ }^{2}-\frac{1}{18} g_{\sigma}{ }^{2} \lambda_{\sigma}{ }^{3} \\
& +\frac{1}{2}(m-1)(m+2)\left(\frac{7}{72} g_{\sigma}{ }^{3} \tilde{\lambda}^{2}-\frac{1}{4} g_{\sigma}{ }^{2} \tilde{\lambda}^{3}+\frac{7}{36} g_{\sigma}{ }^{2} \lambda_{\sigma} \tilde{\lambda}^{2}\right) \\
& -\frac{1}{2 m}(m-1)(m+2)\left(\frac{49}{18} g_{\sigma}^{3}+\frac{37}{18} g_{\sigma}^{2} \tilde{\lambda}+\frac{5}{9} g_{\sigma}^{2} \tilde{\lambda}+\frac{3}{2} g_{\sigma} \tilde{\lambda} \lambda_{\sigma}+\frac{101}{36} g_{\sigma} \tilde{\lambda}^{2}+\frac{1}{4} \tilde{\lambda}^{2} \lambda_{\sigma}-\frac{5}{36} \tilde{\lambda}^{3}\right) g_{\rho}^{2} \\
& +\frac{1}{144 m^{2}}(m-1)(m+2)\left(2\left(7 N-m^{2}-10 m+20\right) \tilde{\lambda}-\left(11 N-5 m^{2}+49 m-98\right) g_{\sigma}\right) g_{\rho}^{4} \\
& -\frac{1}{16 m^{2}}(m-1)(m-2)(m+2)(m+4)\left(g_{\sigma} g_{\rho}^{3} \lambda_{\rho}+3 g_{\rho}{ }^{3} \tilde{\lambda} \lambda_{\rho}+\frac{1}{9} g_{\rho}{ }^{2} \tilde{\lambda} \lambda_{\rho}{ }^{2}-\frac{7}{36} g_{\sigma} g_{\rho}{ }^{2} \lambda_{\rho}^{2}\right), \\
& \bar{\beta}_{g_{\rho}}{ }^{(2)}=-\frac{1}{2} g_{\sigma}{ }^{3} g_{\rho} \lambda_{\sigma}-\frac{3}{2} g_{\sigma}{ }^{2} g_{\rho} \tilde{\lambda} \lambda_{\sigma}-\frac{1}{2} g_{\sigma} g_{\rho} \tilde{\lambda}^{2} \lambda_{\sigma}+\frac{7}{72} g_{\sigma}{ }^{2} g_{\rho} \lambda_{\sigma}{ }^{2}+\frac{7}{36} g_{\sigma} g_{\rho} \tilde{\lambda} \lambda_{\sigma}{ }^{2} \\
& -\frac{1}{2 m}(m-2)(m+4)\left(\frac{5}{18} g_{\sigma}{ }^{2} g_{\rho}^{2} \lambda_{\rho}+\frac{9}{4} g_{\sigma} g_{\rho}^{2} \tilde{\lambda} \lambda_{\rho}+\frac{13}{72} g_{\rho}^{2} \tilde{\lambda}^{2} \lambda_{\rho}+\frac{11}{72} g_{\sigma} g_{\rho} \tilde{\lambda} \lambda_{\rho}^{2}\right) \\
& +\frac{1}{288 m^{2}}(m-2)(m+4)\left(\left(14 N+7 m^{2}-38 m+76\right) g_{\rho}^{4} \lambda_{\rho}-\frac{1}{2}\left(18 m^{2}+101 m-418\right) g_{\rho}^{3} \lambda_{\rho}^{2}\right. \\
& \left.+\frac{1}{4}\left(5 m^{2}-8 m+104\right) g_{\rho}^{2} \lambda_{\rho}^{3}\right) \\
& -\frac{1}{144 m^{2}}\left(N\left(9 m^{2}+11 m-58\right)+22 m^{3}+149 m^{2}-196 m+196\right) g_{\rho}^{5} \\
& -\frac{1}{36 m}\left(18 N+2 m^{2}+49 m-98\right) g_{\sigma}^{2} g_{\rho}^{3}+\frac{7}{72}(N-14) g_{\sigma}^{4} g_{\rho} \\
& +\frac{1}{36 m}\left(7 N-11 m^{2}-47 m+94\right) g_{\sigma} g_{\rho}^{3} \tilde{\lambda}+\frac{1}{36}(7 N-40) g_{\sigma}^{3} g_{\rho} \tilde{\lambda} \\
& -\frac{1}{72 m}\left(18 m^{2}+47 m-94\right) g_{\rho}^{3} \tilde{\lambda}^{2}+\frac{1}{144}\left(7 m^{2}+7 m-446\right) g_{\sigma}{ }^{2} g_{\rho} \tilde{\lambda}^{2} \\
& +\frac{1}{72}\left(7 m^{2}+7 m-22\right) g_{\sigma} g_{\rho} \tilde{\lambda}^{3} \text {, } \\
& \bar{\beta}_{\lambda_{\sigma}}{ }^{(2)}=-\frac{1}{6} N g_{\sigma}{ }^{5}-\frac{9}{4} N g_{\sigma}{ }^{4} \lambda_{\sigma}-\frac{3}{4} N g_{\sigma}{ }^{3} \lambda_{\sigma}{ }^{2}+\frac{7}{24} N g_{\sigma}{ }^{2} \lambda_{\sigma}{ }^{3}-\frac{23}{24} \lambda_{\sigma}{ }^{5} \\
& -n \frac{1}{2}(m-1)(m+2)\left(\frac{1}{6} g_{\sigma}{ }^{3} g_{\rho}{ }^{2}+\frac{9}{4} g_{\sigma}{ }^{2} g_{\rho}{ }^{2} \tilde{\lambda}+\frac{3}{4} g_{\sigma} g_{\rho}{ }^{2} \tilde{\lambda}^{2}-\frac{7}{24} g_{\rho}{ }^{2} \tilde{\lambda}^{3}\right) \\
& -\frac{1}{2}(m-1)(m+2)\left(\frac{1}{6} \tilde{\lambda}^{5}+\frac{9}{4} \tilde{\lambda}^{4} \lambda_{\sigma}+\frac{3}{4} \tilde{\lambda}^{3} \lambda_{\sigma}{ }^{2}-\frac{7}{24} \tilde{\lambda}^{2} \lambda_{\sigma}{ }^{3}\right) \\
& -\frac{23}{192 m}(m-1)(m-2)(m+2)(m+4) \tilde{\lambda}^{3} \lambda_{\rho}^{2},
\end{aligned}
$$




$$
\begin{aligned}
& \bar{\beta}_{\lambda_{\rho}}{ }^{(2)}=-n\left(\frac{1}{6} g_{\rho}{ }^{3} g_{\sigma}{ }^{2}+\frac{9}{2} g_{\rho}{ }^{3} g_{\sigma} \tilde{\lambda}+\frac{3}{4} g_{\rho}{ }^{3} \tilde{\lambda}^{2}+\frac{3}{2} g_{\rho}{ }^{2} g_{\sigma} \tilde{\lambda} \lambda_{\rho}-\frac{7}{12} g_{\rho}{ }^{2} \tilde{\lambda}^{2} \lambda_{\rho}\right) \\
& +\frac{7}{24} N g_{\sigma}{ }^{2} \tilde{\lambda}^{2} \lambda_{\rho}-\frac{5}{2} \tilde{\lambda}^{3} \lambda_{\rho} \lambda_{\sigma}+\frac{7}{24} \tilde{\lambda}^{2} \lambda_{\rho} \lambda_{\sigma}^{2} \\
& +n\left(\frac{1}{24 m}\left(7 m^{2}-2 m+4\right) g_{\rho}^{5}-\frac{3}{16 m}\left(m^{2}+6 m-24\right) g_{\rho}^{4} \lambda_{\rho}\right. \\
& \left.-\frac{3}{32 m}\left(m^{2}+4 m-24\right) g_{\rho}{ }^{3} \lambda_{\rho}^{2}+\frac{7}{192 m}\left(m^{2}+4 m-24\right) g_{\rho}{ }^{2} \lambda_{\rho}{ }^{3}\right) \\
& +\frac{1}{48}\left(7 m^{2}+7 m-282\right) \tilde{\lambda}^{4} \lambda_{\rho}-\frac{1}{96 m}\left(87 m^{2}+304 m-1736\right) \tilde{\lambda}^{2} \lambda_{\rho}^{3} \\
& -\frac{1}{384 m^{2}}\left(m^{4}+33 m^{3}-108 m^{2}-1160 m+3840\right) \lambda_{\rho}^{5}, \\
& \bar{\beta}_{\tilde{\lambda}}^{(2)}=\frac{1}{72} N\left(-18 g_{\sigma}{ }^{3} \tilde{\lambda}^{2}+7 g_{\sigma}{ }^{2} \tilde{\lambda}^{3}+14 g_{\sigma}{ }^{2} \tilde{\lambda}^{2} \lambda_{\sigma}\right) \\
& +n\left(-\frac{1}{6} g_{\sigma}{ }^{3}-3 g_{\sigma}{ }^{2} \tilde{\lambda}-\frac{3}{4} g_{\sigma} \tilde{\lambda}^{2}+\frac{7}{36} \tilde{\lambda}^{3}-\frac{3}{4} g_{\sigma}{ }^{2} \lambda_{\sigma}-\frac{1}{2} g_{\sigma} \tilde{\lambda} \lambda_{\sigma}+\frac{7}{72} \tilde{\lambda}^{2} \lambda_{\sigma}\right) g_{\rho}{ }^{2} \\
& +n \frac{1}{m}\left(\frac{1}{12}\left(2 m^{2}-m+2\right) g_{\sigma}-\frac{1}{8}\left(m^{2}+3 m-6\right) \tilde{\lambda}\right) g_{\rho}^{4} \\
& -\frac{1}{144}\left(11 m^{2}+11 m+174\right) \tilde{\lambda}^{5}+\frac{1}{72}\left(7 m^{2}+7 m-90\right) \tilde{\lambda}^{4} \lambda_{\sigma}-\frac{101}{72} \tilde{\lambda}^{3} \lambda_{\sigma}^{2}-\frac{1}{18} \tilde{\lambda}^{2} \lambda_{\sigma}^{3} \\
& -n \frac{1}{8 m}(m-2)(m+4)\left(3 g_{\rho}^{3} g_{\sigma} \lambda_{\rho}+g_{\rho}^{3} \tilde{\lambda} \lambda_{\rho}+\frac{1}{2} g_{\rho}^{2} g_{\sigma} \lambda_{\rho}^{2}-\frac{7}{12} g_{\rho}^{2} \tilde{\lambda} \lambda_{\rho}^{2}\right) \\
& -\frac{1}{72 m}(m-2)(m+4)\left(67 \tilde{\lambda}^{3} \lambda_{\rho}^{2}+\frac{83}{4} \tilde{\lambda}^{2} \lambda_{\rho}^{2} \lambda_{\sigma}\right) \\
& -\frac{1}{384 m^{2}}(m-2)(m+4)\left(11 m^{2}+46 m-280\right) \tilde{\lambda} \lambda_{\rho}^{4} .
\end{aligned}
$$

For the anomalous dimensions

$$
\begin{aligned}
& \gamma_{\Phi}^{(2)}=-\frac{1}{432}(11 N-26) g_{\sigma}{ }^{4}+\frac{1}{9} g_{\sigma}{ }^{3} \lambda_{\sigma}-\frac{11}{432} g_{\sigma}{ }^{2} \lambda_{\sigma}{ }^{2} \\
& +\frac{1}{864 m}(m-1)(m+2)\left(-11 m g_{\sigma}{ }^{2} \tilde{\lambda}^{2}+52 g_{\rho}^{2} g_{\sigma}^{2}-22 g_{\rho}^{2} \tilde{\lambda}^{2}+144 g_{\rho}^{2} g_{\sigma} \tilde{\lambda}\right) \\
& +\frac{1}{3456 m^{2}}(m-1)(m-2)(m+2)(m+4)\left(48 g_{\rho}^{3} \lambda_{\rho}-11 g_{\rho}^{2} \lambda_{\rho}^{2}\right) \\
& -\frac{1}{864 m^{2}}(m-1)(m+2)\left(11 N+11 m^{2}-13 m+26\right) g_{\rho}^{4}, \\
& \gamma_{\sigma}^{(2)}=\frac{1}{9}\left(N\left(\frac{1}{24} g_{\sigma}{ }^{4}+g_{\sigma}{ }^{3} \lambda_{\sigma}-\frac{11}{48} g_{\sigma}{ }^{2} \lambda_{\sigma}{ }^{2}\right)+\frac{13}{48} \lambda_{\sigma}{ }^{4}\right) \\
& +\frac{1}{18}(m-1)(m+2)\left(n\left(\frac{1}{24} g_{\sigma}^{2} g_{\rho}^{2}+g_{\sigma} g_{\rho}^{2} \tilde{\lambda}-\frac{11}{48} g_{\rho}^{2} \tilde{\lambda}^{2}\right)+\frac{1}{24} \tilde{\lambda}^{4}+\tilde{\lambda}^{3} \lambda_{\sigma}-\frac{11}{48} \tilde{\lambda}^{2} \lambda_{\sigma}^{2}\right) \\
& +\frac{13}{3456 m}(m-1)(m-2)(m+2)(m+4) \tilde{\lambda}^{2} \lambda_{\rho}^{2}, \\
& \gamma_{\rho}^{(2)}=\frac{1}{432} n\left(2 g_{\sigma}{ }^{2} g_{\rho}^{2}+96 g_{\rho}^{2} g_{\sigma} \tilde{\lambda}-11 g_{\rho}^{2} \tilde{\lambda}^{2}-11 m g_{\sigma}{ }^{2} \tilde{\lambda}^{2}\right)+\frac{1}{9} \tilde{\lambda}^{3} \lambda_{\sigma}-\frac{11}{432} \tilde{\lambda}^{2} \lambda_{\sigma}{ }^{2} \\
& -\frac{1}{432 m}\left(11 m^{2}-m+2\right) n g_{\rho}^{4}-\frac{1}{864}\left(11 m^{2}+11 m-74\right) \tilde{\lambda}^{4}
\end{aligned}
$$




$$
\begin{aligned}
& +\frac{1}{1728 m}(m-2)(m+4)\left(n\left(48 g_{\rho}^{3} \lambda_{\rho}-11 g_{\rho}{ }^{2} \lambda_{\rho}^{2}\right)+87 \tilde{\lambda}^{2} \lambda_{\rho}^{2}\right) \\
& +\frac{1}{6912 m^{2}}(m-2)(m+4)\left(m^{2}+26 m-200\right) \lambda_{\rho}^{4} .
\end{aligned}
$$

The corresponding three-loop results, obtained using (2.5), for the anomalous dimensions and $\beta$-functions of the theory defined by (4.33) are included in an ancillary file.

When $m<4$ there are fixed points with just $\lambda_{\rho}$ non zero

$$
\lambda_{\rho *}{ }^{2}=\frac{8 m}{(m+10)(4-m)} \varepsilon, \quad\left(\begin{array}{ll}
\partial_{\lambda_{\rho}} \beta_{\lambda_{\rho}} & \partial_{\lambda_{\rho}} \beta_{g_{\rho}} \\
\partial_{g_{\rho}} \beta_{\lambda_{\rho}} & \partial_{g_{\rho}} \beta_{g_{\rho}}
\end{array}\right)=\left(\begin{array}{cc}
\varepsilon & 0 \\
0 & \frac{m^{2}+5 m-32}{6(m+10)(4-m)} \varepsilon
\end{array}\right) .
$$

Fixed points determined by vanishing of the $\beta$-functions for the couplings $g_{\sigma}, g_{\rho}, \lambda_{\sigma}, \lambda_{\rho}, \tilde{\lambda}$ belong to equivalence classes as a consequence of (4.36). For large $n$, to leading order in $1 / n$, there are three inequivalent fixed points similar to those in the $O(N)$ model where the non zero couplings are $\mathrm{O}\left(n^{-\frac{1}{2}}\right)$. To $\mathrm{O}\left(\varepsilon^{2}\right)$ these are given by

$$
\begin{aligned}
& \text { H) } g_{\rho *}=\lambda_{\rho *}=\tilde{\lambda}_{*}=0, \quad g_{\sigma *}^{2}=\frac{6}{N} \varepsilon\left(1+\frac{44}{N}-\frac{155}{3 N} \varepsilon\right), \quad \lambda_{\sigma *}^{2}=\frac{216}{N} \varepsilon\left(1+\frac{324}{N}-\frac{215}{N} \varepsilon\right), \\
& \text {-) } g_{\sigma *}=\lambda_{\sigma *}=\tilde{\lambda}_{*}=0 \text {, } \\
& g_{\rho *}^{2}=\frac{6}{n} \varepsilon\left(1+\frac{1}{N}\left(7 m^{2}+22 m-80\right)-\frac{1}{6 N}\left(50 m^{2}+155 m-598\right) \varepsilon\right) \text {, } \\
& \lambda_{\rho *}^{2}=\frac{216}{n} \varepsilon\left(1+\frac{27}{N}(m-4)(m+10)-\frac{1}{2 N}\left(50 m^{2}+215 m-1726\right) \varepsilon\right), \\
& \text { +) } \quad g_{\sigma *}^{2}=\frac{6}{N} \varepsilon\left(1+\frac{1}{n}(m+1)\left(22-\frac{155}{6} \varepsilon\right)\right), \quad \lambda_{\sigma *}^{2}=\frac{216}{N} \varepsilon\left(1+\frac{1}{n}(m+1)\left(162-\frac{215}{2} \varepsilon\right)\right) \text {, } \\
& \tilde{\lambda}_{*}^{2}=\frac{216}{N} \varepsilon\left(1+\frac{1}{n}\left(18(4 m+9)-\frac{5}{2}(21 m+43) \varepsilon\right)\right), \\
& g_{\rho *}^{2}=\frac{6}{n} \varepsilon\left(1+\frac{1}{n}\left(7 m+22-\frac{5}{6}(10 m+31) \varepsilon\right)\right) \text {, } \\
& \lambda_{\rho *}^{2}=\frac{216}{n} \varepsilon\left(1+\frac{1}{n}\left(27(m+6)-\frac{5}{2}(10 m+43) \varepsilon\right)\right) \text {. }
\end{aligned}
$$

The eigenvalues of the stability matrix to first order in $\varepsilon$ at large $n$ are then

$$
\begin{aligned}
& \text { H) }\left(1,1-\frac{420}{N}, \frac{40}{N},-\frac{1}{2},-\frac{1}{2}-\frac{4}{N}\right) \varepsilon, \\
& \text {-) }\left(1,1-\frac{30}{N}\left(m^{2}+7 m-15\right), \frac{1}{2}-\frac{2}{N}\left(10 m^{2}+25 m-122\right),-\frac{1}{2},-\frac{1}{2}-\frac{20}{N}(m-1)(m+2)\right) \varepsilon, \\
& \text { +) }\left(1,1+\frac{40}{n}(m+1), 1+\frac{10}{N} x_{1}, 1+\frac{10}{N} x_{2}, 1+\frac{10}{N} x_{3}\right) \varepsilon, \\
& f\left(x_{i}\right)=0, f(x)=x^{3}-3 m(m-6) x^{2}-9 m^{2}\left(2 m^{2}+17 m+99\right) x-54 m^{3}\left(m^{2}-55 m-2\right) .
\end{aligned}
$$

Clearly the last fixed point is stable and there are in general three real roots, for large $m$, $x_{1} \approx-3 m^{2}, x_{2} \approx-3 m, x_{3} \approx 6 m^{2}$. The corresponding leading non zero contributions to 
the anomalous dimensions for each case are given by, to order $1 / n$ and $\mathrm{O}\left(\varepsilon^{3}\right)$ from three loops, using (2.5),

$$
\gamma_{\Phi H,+,-}=k_{H,+,-} \frac{1}{n}\left(\varepsilon-\frac{11}{12} \varepsilon^{2}-\frac{13}{144} \varepsilon^{3}\right)
$$

where

$$
k_{H}=\frac{1}{m}, \quad k_{-}=\frac{1}{2 m}(m-1)(m+2), \quad k_{+}=\frac{1}{2}(m+1) .
$$

The anomalous dimensions for $\sigma, \rho$ in the same limit are

$$
\begin{aligned}
H) \gamma_{\sigma H} & =\frac{1}{2} \varepsilon+k_{H} \frac{8}{n}\left(5 \varepsilon-\frac{13}{3} \varepsilon^{2}-\frac{11}{36} \varepsilon^{3}\right) \\
-) \gamma_{\rho-} & =\frac{1}{2} \varepsilon-\gamma_{\Phi-}+\frac{1}{N}(m-2)\left(3(3 m+13) \varepsilon-\frac{1}{4}(33 m+139) \varepsilon^{2}-\frac{1}{16}(5 m+31) \varepsilon^{3}\right) \\
+) \quad \gamma_{\sigma+} & =\frac{1}{2} \varepsilon+k_{+} \frac{8}{n}\left(5 \varepsilon-\frac{13}{3} \varepsilon^{2}-\frac{11}{36} \varepsilon^{3}\right) \\
\gamma_{\rho+} & =\frac{1}{2} \varepsilon-\gamma_{\Phi+}+\frac{1}{n}\left(3(3 m+7) \varepsilon-\frac{1}{4}(33 m+73) \varepsilon^{2}-\frac{1}{16}(5 m+21) \varepsilon^{3}\right)
\end{aligned}
$$

These results are in accord with large $n$ expansions [30-32]. The operators $\sigma, \rho$ have canonical dimension $\frac{1}{2}(d-2)$ and the $\frac{1}{2} \varepsilon$ terms in (4.46) ensure that they have leading dimension 2 at large $n$.

\section{Scalar theories with reduced symmetry in $4-\varepsilon$ dimensions}

For $N$-flavour scalar theories in $4-\varepsilon$ dimensions the fixed point structure even with just two essential couplings can be rather non trivial. Many such theories with different symmetry groups have been discussed in the literature [33]. A large class can be encompassed by considering a quartic potential with two couplings of the form

$$
V(\phi)=\frac{1}{24} \lambda_{i j k l} \phi_{i} \phi_{j} \phi_{k} \phi_{l}=\frac{1}{8} \lambda\left(\phi^{2}\right)^{2}+\frac{1}{24} g d_{i j k l} \phi_{i} \phi_{j} \phi_{k} \phi_{l},
$$

where $d_{i j k l}$ is symmetric and traceless on any pair of indices and is assumed to be the unique tensor invariant under some subgroup $H \subset O(N)$ (in general there are, for arbitrary $N$, $\frac{1}{24}(N-1) N(N+1)(N+6)$ such symmetric traceless tensors). For $g=0$ this reduces to the usual $O(N)$ symmetric theory and the extra term breaks $O(N)$ to the subgroup $H$. To first order in $g(5.1)$ describes a perturbation of the $O(N)$ symmetric theory by an approximately marginal harmonic operator [34]. In general there are bounds such that $-\delta_{-}\left(\phi^{2}\right)^{2} \leq d_{i j k l} \phi_{i} \phi_{j} \phi_{k} \phi_{l} \leq \delta_{+}\left(\phi^{2}\right)^{2}, \delta_{ \pm}>0$ for $N \geq 2$, which lead to constraints on $\lambda, g$ to ensure the potential is bounded below. For $\lambda$ positive then for $g>0$ stability requires $3 \lambda / g>\delta_{-}$while for $g<0,-3 \lambda / g>\delta_{+}$.

A general quartic potential would contain a term $\frac{1}{4} \phi^{2} d_{i j} \phi^{i} \phi^{j}$, with $d_{i j}$ symmetric and traceless, in addition to (5.1). For cases considered here the only quadratic in $\phi$ invariant under $H$ is $\phi^{2}$ and so such terms can be dropped. The restriction to (5.1) does not exclude any fixed points relevant for condensed matter systems considered in the literature but an 
example in which the perturbation is extended by a further coupling and where the RG flow realises new IR fixed points is described in appendix B.

To two loop order it is sufficient to ensure the RG flow is restricted to the two dimensional space parameterised by $\lambda, g$ to impose

$$
d_{i j m n} d_{k l m n}=\frac{1}{N-1} a\left(\frac{1}{2} N\left(\delta_{i k} \delta_{j l}+\delta_{i l} \delta_{j k}\right)-\delta_{i j} \delta_{k l}\right)+b d_{i j k l}
$$

Tensors $d_{i j k l}$ related by $O(N)$ transformations define equivalent theories, manifestly $H$ always contains $\mathbb{Z}_{2}$ induced by $\phi_{i} \rightarrow-\phi_{i}$ for all $i$. For any non zero $d_{i j k l}, a>0$ and we may choose $b>0$ by changing the sign of $d_{i j k l}$ if necessary, the value of $b^{2} / a$ is independent of the choice of normalisation. As a consequence of (5.2) $d_{i k l m} d_{j k l m}=\frac{1}{2}(N+2) a \delta_{i j}$, as expected if the only $H$ invariant quadratic in $\phi$ is $\phi^{2}$. There is then only one strongly relevant $\mathbb{Z}_{2}$ invariant operator which needs to be tuned to zero to attain an IR fixed point under RG flow. For (5.2) the subgroup $H$ is discrete. More general possibilities than (5.2) allowing continuous $H$ and also more than one such tensor are considered later.

For $N=2(5.2)$ requires $b=0$, which can be seen by taking $d_{1111}=d_{2222}=-d_{1122}=$ $1, d_{1112}=-d_{1222}=d$, so that $d_{i j k l} \phi_{i} \phi_{j} \phi_{k} \phi_{l}=\frac{1}{2}(1-i d)\left(\phi_{1}+i \phi_{2}\right)^{4}+\frac{1}{2}(1+i d)\left(\phi_{1}-i \phi_{2}\right)^{4}$, and then (5.2) requires $a=2\left(d^{2}+1\right)$ as well as $b=0 .{ }^{3}$ For $N>2$ there is an inequality obtained in appendix $\mathrm{C}$

$$
a \geq 2 \frac{N-1}{(N-2)^{2}} b^{2} .
$$

For $N=3$ the inequality is saturated so that $a=4 b^{2}$. Possible solutions for $a, b$ are further constrained by considering the eigenvalue problem

$$
d_{i j k l} v_{k l}=\mu v_{i j}, v_{i j}=v_{j i}, v_{i i}=0 \Rightarrow \mu^{2}-b \mu-\frac{N}{N-1} a=0,
$$

from (5.2). For solutions $\mu_{ \pm}$then for $d_{+}$eigenvectors with eigenvalue $\mu_{+}$there must be $d_{-}=\frac{1}{2}(N-1)(N+2)-d_{+}$with eigenvalue $\mu_{-}$. The traceless condition then leads, as in the discussion in section 4 , to

$$
1+\frac{4 N}{N-1} \frac{a}{b^{2}}=\left(\frac{\frac{1}{2}(N-1)(N+2)}{\frac{1}{2}(N-1)(N+2)-2 d_{+}}\right)^{2} \geq \frac{(N+2)^{2}}{(N-2)^{2}},
$$

using the inequality (5.3). This requires $d_{ \pm}$are restricted to the finite range

$$
N-1 \leq d_{ \pm} \leq \frac{1}{2} N(N-1)
$$

The spaces of dimension $d_{ \pm}$must form representations of $H$. Tensors $d_{i j k l}$ are equivalent under $d_{+} \leftrightarrow d_{-}$. Thus for $N=3$ there is just one case when $d_{ \pm}=2, d_{\mp}=3$, for $N=4$ there are two possibilities $d_{ \pm}=3, d_{\mp}=6$ and $d_{ \pm}=4, d_{\mp}=5$.

\footnotetext{
${ }^{3}$ The results reduce to the two flavour theory in section 5 with $\lambda_{1}=\lambda_{2}=3 \lambda+g, \lambda_{0}=\lambda-g, g_{1}=$ $-g_{2}=d g$. In terms of (3.9) case a) corresponds to taking $g^{2}=\left(d^{2}+1\right) g^{2}$ and the $O(2)$ theory of course requires $g=0$.
} 
For the general scalar theory the lowest order $\beta$-function is determined, after rescaling $\lambda_{i j k l} \rightarrow(4 \pi)^{2} \lambda_{i j k l}$, by the gradient flow equation [17]

$$
\beta_{i j k l}=\frac{\partial}{\partial \lambda_{i j k l}} A, \quad A=-\frac{1}{2} \varepsilon \lambda_{i j k l} \lambda_{i j k l}+\lambda_{i j k l} \lambda_{k l m n} \lambda_{m n i j},
$$

and at any fixed point at this order [16]

$$
A_{*}=-\frac{1}{6} \varepsilon \lambda_{* i j k l} \lambda_{* i j k l}=-2 \varepsilon \operatorname{tr}\left(\gamma_{*}\right),
$$

with $\gamma_{*}$ the lowest order $\phi$ anomalous dimension matrix at the fixed point as given in (3.6). For positive $\lambda_{i j k l} A$ is bounded below so that $A$ has at least one non zero perturbative minimum in the $\varepsilon$ expansion so long as the RG flow does not destabilise the vacuum.

With the constraint (5.2) the lowest order $\beta$-functions become

$$
\begin{aligned}
& \beta_{\lambda}=-\varepsilon \lambda+(N+8) \lambda^{2}+a g^{2}, \\
& \beta_{g}=-\varepsilon g+12 \lambda g+3 b g^{2} .
\end{aligned}
$$

The associated $a$-function is given by specialising (5.7)

$$
A=N(N+2)\left(-\frac{3}{2} \varepsilon \lambda^{2}-\frac{1}{4} \varepsilon a g^{2}+(N+8) \lambda^{3}+3 a \lambda g^{2}+\frac{1}{2} a b g^{3}\right),
$$

so that, with the $\beta$-functions in (5.9),

$$
\frac{\partial}{\partial \lambda} A=3 N(N+2) \beta_{\lambda}, \quad \frac{\partial}{\partial g} A=\frac{1}{2} N(N+2) a \beta_{g} .
$$

To lowest order in the $O(N)$ invariant theory, obtained when $g=0$, the Heisenberg fixed point is given by

$$
\lambda_{* H}=\frac{1}{N+8} \varepsilon, \quad A_{* H}=-\frac{N(N+2)}{2(N+8)^{2}} \varepsilon^{3}=\frac{(N-4)^{2}}{48(N+8)^{2}} \varepsilon^{3}-\frac{1}{48} N \varepsilon^{3} .
$$

For theories described by the lowest order $\beta$-functions in (5.9) there are potentially, besides the trivial Gaussian fixed point and the $O(N)$ symmetric fixed point with $g=0$, two additional fixed points. To analyse these fixed points it is convenient to write in (5.9) $\beta_{\lambda}=-\varepsilon \lambda+\lambda^{2} f_{\lambda}(x), \beta_{g}=-\varepsilon g+\lambda^{2} f_{g}(x)$ for $x=g / \lambda$ and $f_{\lambda}(x)=N+8+a x^{2}, f_{g}(x)=$ $12 x+3 b x^{2}$. Then the fixed point condition requires

$$
f(x)=0 \quad \text { for } \quad f(x)=x f_{\lambda}(x)-f_{g}(x)=x\left(a x^{2}-3 b x+N-4\right) .
$$

The cubic polynomial $f(x)$ has simultaneous roots for $N=4$ or $b^{2} / a=\frac{4}{9}(N-4)$. The solution $x=0$ of course corresponds to the $O(N)$ symmetric theory with $g=0$. For $g=0$ $\lambda_{*}=\varepsilon /(N+8)$ but if $N>4 g$ is the coupling to a relevant operator. So long as

$$
F(N)=16-4 N+9 b^{2} / a>0,
$$


(the bound $(5.3)$ gives $F(N)<\frac{(N+2)^{2}}{2(N-1)}$ ) there are two extra real fixed points determined by the roots

$$
\sqrt{a} x_{ \pm}=X_{ \pm}, \quad X_{ \pm}=\frac{1}{2}\left(3 b / \sqrt{a} \pm F(N)^{\frac{1}{2}}\right)=\frac{1}{2}\left((4(N-4)+F(N))^{\frac{1}{2}} \pm F(N)^{\frac{1}{2}}\right) .
$$

To leading order in $\varepsilon$ the additional fixed points are given by

$$
\lambda_{* \pm}=\frac{1}{N+8+X_{ \pm}^{2}} \varepsilon, \quad \sqrt{a} g_{* \pm}=\frac{X_{ \pm}}{N+8+X_{ \pm}^{2}} \varepsilon,
$$

For the stability matrix

$$
M=\left.\left(\begin{array}{ll}
\partial_{\lambda} \beta_{\lambda} & \partial_{g} \beta_{\lambda} \\
\partial_{\lambda} \beta_{g} & \partial_{g} \beta_{g}
\end{array}\right)\right|_{\lambda=\lambda_{*}, g=g_{*}},
$$

then for either $x_{*}=x_{ \pm}$or $x_{*}=0$

$$
\operatorname{det} M=-\varepsilon \lambda_{*} f^{\prime}\left(x_{*}\right), \quad \operatorname{tr} M=\varepsilon-\lambda_{*} f^{\prime}\left(x_{*}\right), \quad x_{*}=g_{*} / \lambda_{*}, \quad f\left(x_{*}\right)=0 .
$$

At a fixed point $\lambda_{*}=\varepsilon / f_{\lambda}\left(x_{*}\right)$. From (5.18) the eigenvalues of $M$ to first order in $\varepsilon$ are just

$$
\kappa_{0}=\varepsilon, \quad \kappa_{1}=-\lambda_{*} f^{\prime}\left(x_{*}\right) .
$$

just as in (5.19). In consequence the fixed point is stable if $f^{\prime}\left(x_{*}\right)<0$. Since $a>0$ there is one possible root $x_{*}$ of the cubic $f(x)$ with negative $f^{\prime}\left(x_{*}\right)$ and two which are positive. For $N<4$ the $O(N)$ symmetric root at $x=0$, giving $\lambda_{*}=\varepsilon /(N+8), g_{*}=0$, has $f^{\prime}(0)=$ $N-4<0$ and so is stable. The other roots corresponding to non zero $g_{*}$ are then unstable. Otherwise $f^{\prime}(0)>0$ and $f^{\prime}\left(x_{ \pm}\right) \gtrless 0$, assuming $b>0$. The perturbation is then always relevant when $N>4$ and leads to an RG flow along which the $O(N)$ symmetry is broken.

Applying (5.16) in (5.10) gives

$$
A_{* \pm}=-\frac{1}{2} N(N+2) \frac{1+\frac{1}{6} X_{ \pm}^{2}}{\left(N+8+X_{ \pm}^{2}\right)^{2}} \varepsilon^{3} \geq-\frac{1}{48} N \varepsilon^{3} .
$$

The bound arises when

$$
X_{ \pm}^{2}=N-4 \quad \Rightarrow \quad F(N)=0 .
$$

The bound is attained at the Heisenberg fixed point (5.12) when $N=4$. In general $F(N)$ vanishes when the fixed points merge, for small $F$

$$
A_{* \pm}=-\frac{1}{48} N\left(1-\frac{N-4}{4(N+2)^{2}} F(N) \mp \frac{3 \sqrt{N-4}}{2(N+2)^{3}} F(N)^{\frac{3}{2}}+\mathrm{O}\left(F(N)^{2}\right)\right) \varepsilon^{3}
$$

so that for $N>4$ the least $A_{*}$, and a stable fixed point, is expected to be achieved for the smallest $F(N)$ and for the fixed point correspond to $X_{-}$, as observed above. In general for $N>4, A_{*, H}>A_{* \pm}$.

At two loops

$$
\begin{aligned}
& \beta_{\lambda}^{(2)}=-3(3 N+14) \lambda^{3}-\frac{1}{6}(5 N+82) a \lambda g^{2}-2 a b g^{3}, \\
& \beta_{g}^{(2)}=-(5 N+82) \lambda^{2} g+\frac{1}{6(N-1)}\left(N^{2}-17 N+34\right) a g^{3}-6 b\left(6 \lambda g^{2}+b g^{3}\right),
\end{aligned}
$$


and the anomalous dimension matrix is $\gamma \mathbb{1}$ with

$$
\gamma^{(2)}=\frac{1}{4}(N+2)\left(\lambda^{2}+\frac{1}{6} a g^{2}\right)
$$

and from (5.10) to lowest order in $\varepsilon$

$$
A_{*}=\left.A\right|_{\beta_{\lambda}=\beta_{g}=0}=-2 N \gamma_{*}^{(2)} \varepsilon .
$$

At the Heisenberg fixed point given by (5.12)

$$
\gamma_{H}=\frac{N+2}{4(N+8)^{2}} \varepsilon^{2} .
$$

At three loops results further conditions are necessary to restrict the RG flow to two couplings. In addition to (5.2) we require

$$
\begin{aligned}
d_{i j k p} d_{i l m q} d_{l j n r} d_{n m k s} & =\frac{1}{4} \mathcal{A}\left(\delta_{p q} \delta_{r s}+\delta_{p r} \delta_{q s}+\delta_{p s} \delta_{q r}\right)+c d_{p q r s}, \\
\mathcal{A} & =\frac{1}{N-1} a\left((N-2) a+2(N-1) b^{2}\right),
\end{aligned}
$$

where $c^{2} / a^{3}$, or $c / b^{3}$, does not depend on the arbitrary normalisation of $d_{i j k l}$. This gives for the three loop $\beta$-functions

$$
\begin{aligned}
\beta_{\lambda}^{(3)}= & \frac{1}{8}\left(33 N^{2}+922 N+2960\right) \lambda^{4}+12(5 N+22) \zeta_{3} \lambda^{4} \\
& +\frac{1}{16}\left(N^{2}+500 N+3492\right) a \lambda^{2} g^{2}+12(N+14) \zeta_{3} a \lambda^{2} g^{2} \\
& +\frac{1}{8}(27 N+470) a b \lambda g^{3}+48 \zeta_{3} a b \lambda g^{3} \\
& -\frac{1}{16(N-1)}\left(7 N^{2}-33 N+114\right) a^{2} g^{4}+\frac{13}{2} a b^{2} g^{4}+3 \mathcal{A} \zeta_{3} g^{4}, \\
\beta_{g}^{(3)}= & -\frac{1}{4}\left(13 N^{2}-368 N-3284\right) \lambda^{3} g+48(N+14) \zeta_{3} \lambda^{3} g \\
& +\frac{3}{8}(43 N+1334) b \lambda^{2} g^{2}+432 \zeta_{3} b \lambda^{2} g^{2} \\
& +\frac{3}{N-1}\left(3 N^{2}+33 N-50\right) a \lambda g^{3}+156 b^{2} \lambda g^{3}+72 \mathcal{A} / a \zeta_{3} \lambda g^{3} \\
& -\frac{1}{16(N-1)}\left(11 N^{2}-289 N+626\right) a b g^{4}+\frac{39}{2} b^{3} g^{4}+12 \zeta_{3} c g^{4},
\end{aligned}
$$

and

$$
\gamma^{(3)}=-\frac{1}{16}(N+2)(N+8) \lambda^{3}-\frac{1}{32}(N+2)\left(6 a \lambda g^{2}+a b g^{3}\right) .
$$

The two and three loop results for $\beta$-functions determine corrections as an expansion in $\varepsilon$ to the structure of fixed points found at one loop. The perturbation given by the coupling $g$ in (5.1) becomes relevant in the $O(N)$ symmetric theory, and so induces an RG flow in which the $O(N)$ symmetry is broken, when $\left.\partial_{g} \beta_{g}\right|_{\lambda=\lambda_{*}, g=0}<0$. With results to three loops this requires

$$
N>N_{\text {crit }, H}=4-2 \varepsilon+\frac{5}{2}\left(\zeta_{3}-\frac{1}{6}\right) \varepsilon^{2} .
$$


The results in (5.12) may be straightforwardly extended to higher order, for large $N$ they simplify to

$$
\lambda_{* H} \sim \frac{1}{N} \varepsilon-\frac{1}{N^{2}}\left(8 \varepsilon-9 \varepsilon^{2}+\frac{33}{8} \varepsilon^{3}\right)
$$

leading to

$$
\begin{aligned}
\gamma_{H} & \sim \frac{1}{4 N}\left(\varepsilon^{2}-\frac{1}{4} \varepsilon^{3}\right), \quad \gamma_{\sigma H} \sim \varepsilon-\frac{2}{N}\left(3 \varepsilon-\frac{13}{4} \varepsilon^{2}+\frac{3}{16} \varepsilon^{3}\right), \\
\left.\partial_{g} \beta_{g}\right|_{\lambda=\lambda_{* H}, g=0} & \sim-\varepsilon+\frac{1}{N}\left(12 \varepsilon-5 \varepsilon^{2}-\frac{13}{4} \varepsilon^{3}\right) .
\end{aligned}
$$

In the two coupling theory considered here the broken symmetry fixed points merge at critical point $N=N_{\text {crit }}$. To lowest order in an $\varepsilon$-expansion $N_{\text {crit }}=N_{0}$ with $N_{0}$ determined by $F\left(N_{0}\right)=0$, with $F$ is defined in (5.14). In the neighbourhood of the critical point, $N \approx N_{\text {crit }}$, the straightforward $\varepsilon$-expansion breaks down but if $F(N)$ has a simple zero at $N=N_{0}$ we can write for $N>4, N \approx N_{0}$,

$$
\begin{aligned}
\lambda_{*+}-\lambda_{*-} & =a_{\lambda}(N, \varepsilon) \tilde{F}(N, \varepsilon)^{\frac{1}{2}}(1+\mathrm{O}(\tilde{F})), \\
g_{*+}-g_{*-} & =a_{g}(N, \varepsilon) \tilde{F}(N, \varepsilon)^{\frac{1}{2}}(1+\mathrm{O}(\tilde{F})), \\
\tilde{F}(N, \varepsilon) & =F(N)-F_{1}(N) \varepsilon-F_{2}(N) \varepsilon^{2}+\mathrm{O}\left(\varepsilon^{3}\right),
\end{aligned}
$$

where $F_{1}, F_{2}$ are determined by ensuring that any singular terms in the $\varepsilon$-expansion as $\tilde{F} \rightarrow 0$ are cancelled. Including higher loop corrections the critical $N$ is then determined by iteratively solving

$$
\tilde{F}\left(N_{\text {crit }}, \varepsilon\right)=0, \quad N_{\text {crit }}=N_{0}+\mathrm{O}(\varepsilon)
$$

as an expansion in $\varepsilon$. For fixed points to be present $\tilde{F}(N, \varepsilon)>0$, for $N>N_{\text {crit }, H}$ and $\tilde{F}(N, \varepsilon)<0$ the RG flow does attain fixed points accessible in the $\varepsilon$-expansion. Using the two and three loop results for the $\beta$ functions ${ }^{4}$

$$
\begin{aligned}
F_{1}(N)= & \frac{2(N+2)^{2}}{3(N-1)} \\
F_{2}(N)= & \frac{(N+2)^{2}(2 N+7)}{36(N-1)^{2}} \\
& +\left(\frac{8 N^{4}-155 N^{3}+534 N^{2}-932 N-184}{3(N-1)(N+2)^{2}}-\frac{32(N-4)^{3}}{9(N+2)^{2}} \frac{c}{b^{3}}\right) \zeta_{3} .
\end{aligned}
$$

The general theory described by (5.1) may be extended to include relevant operators quadratic in $\phi$ by letting

$$
V(\phi) \rightarrow V(\phi)+\frac{1}{2} \sigma \phi^{2}+\frac{1}{2} \rho_{i j} \phi_{i} \phi_{j}, \quad \rho_{i i}=0 .
$$

\footnotetext{
${ }^{4}$ Here $a_{\lambda}=-\sqrt{N-4} \varepsilon / 2(N+2)^{2}+\mathrm{O}\left(\varepsilon^{2}\right), \sqrt{a} a_{g}=3 \varepsilon /(N+2)^{2}+\mathrm{O}\left(\varepsilon^{2}\right)$.
} 
At a fixed point the couplings of such operators must be tuned to zero but the general formula for $\beta_{V}(\phi)$, obtained from (2.2) and (3.6), has contributions linear in $\sigma, \rho$ which take the form

$$
\begin{aligned}
\beta_{\sigma}^{(1)}= & (N+2) \lambda \sigma, \quad \beta_{\sigma}^{(2)}=-\frac{5}{2}(N+2)\left(\lambda^{2}+\frac{1}{6} a g^{2}\right) \sigma \\
\beta_{\sigma}^{(3)}= & \frac{1}{16}(N+2)\left(12(5 N+37) \lambda^{3}+(N+164) a g^{2} \lambda+27 a b g^{3}\right) \sigma \\
\beta_{\rho, i j}{ }^{(1)}= & 2 \lambda \rho_{i j}+g d_{i j k l} \rho_{k l}, \\
\beta_{\rho, i j}{ }^{(2)}= & -\frac{1}{2}\left((N+10) \lambda^{2}-\frac{N^{2}-5 N+10}{6(N-1)} a g^{2}\right) \rho_{i j}-\left(4 \lambda g+b g^{2}\right) d_{i j k l} \rho_{k l}, \\
\beta_{\rho, i j}{ }^{(3)}= & -\frac{1}{4}\left(\frac{1}{2}\left(5 N^{2}-84 N-444\right) \lambda^{3}-\frac{5 N^{2}+65 N-82}{N-1} a g^{2} \lambda+\frac{N^{2}-35 N+54}{4(N-1)} a b g^{3}\right) \rho_{i j} \\
& +\frac{1}{8}\left(3(9 N+146) g \lambda^{2}+192 b g^{2} \lambda-\frac{3 N^{2}-25 N+66}{2(N-1)} a g^{3}+32 b^{2} g^{3}\right) d_{i j k l} \rho_{k l} .
\end{aligned}
$$

In general $\beta_{\sigma}=\gamma_{\sigma} \sigma$. The anomalous dimensions for $\rho$ are then determined by the eigenvalues in (5.4).

For application here it is necessary to consider possible tensors $d_{i j k l}$ satisfying (5.2) as well as (5.27). If $b=0$ solving the fixed point equations at lowest order using (5.9) requires $N<4$ or $g=0$.

\subsection{Fixed points with discrete $H \subset O(N)$}

\subsubsection{Hypercubic fixed poins}

An extension of the simple $N=2$ case is obtained by taking, as was considered long ago [35-37] and more recently in [38],

$$
d_{i j k l} \phi_{i} \phi_{j} \phi_{k} \phi_{l}=\sum_{i} \phi_{i}^{4}-\frac{3}{N+2}\left(\phi^{2}\right)^{2}
$$

where $-\frac{2(N-1)}{N(N+2)}\left(\phi^{2}\right)^{2} \leq d_{i j k l} \phi_{i} \phi_{j} \phi_{k} \phi_{l} \leq \frac{N-1}{N+2}\left(\phi^{2}\right)^{2}$. This breaks $O(N)$ to the discrete hypercubic group $H_{\text {cubic }} \simeq B C_{N} \simeq \mathcal{S}_{N} \ltimes \mathbb{Z}_{2}^{N}$, which ensures no other couplings than $\lambda, g$ are necessary for renormalisability. If $\lambda=\frac{1}{N+2} g$ in (5.1) this reduces to $N$ decoupled $\phi^{4}$ theories. In (5.2) and (5.27)

$$
a=2 \frac{N-1}{(N+2)^{2}}, \quad b=\frac{N-2}{N+2}, \quad c=\frac{N(N-2)(N-4)}{(N+2)^{3}},
$$

and the solutions of (5.4) give

$$
\mu_{+}=\frac{N}{N+2}, \quad d_{+}=N-1, \quad \mu_{-}=-\frac{2}{N+2}, \quad d_{-}=\frac{1}{2} N(N-1) .
$$

Clearly (5.39) saturates the inequality (5.3). In (5.14)

$$
F(N)_{\text {cubic }}=\frac{(N+2)^{2}}{2(N-1)}
$$

which is positive for any $N>1$. 
For this case the roots of $f(x)=0$ are $x_{-}=\frac{1}{2}(N-4)(N+2) /(N-1), x_{+}=N+2$ as well as $x=0$. At these roots $f^{\prime}\left(x_{ \pm}\right)= \pm x_{ \pm}$and $f^{\prime}(0)=N-4$. Thus for $N>4 x_{*}=x_{-}$ is the stable fixed point, for $N<4 x_{*}=0$. At lowest order there are then fixed points for $N>1$ for $x_{*}=x_{-}, x_{*}=x_{+}$respectively

$$
\begin{aligned}
& \lambda_{*-}=\frac{2(N-1)}{3 N(N+2)} \varepsilon, \quad g_{*-}=\frac{1}{3 N}(N-4) \varepsilon, \quad \gamma_{*-}=\frac{(N-1)(N+2)}{108 N^{2}} \varepsilon^{2}, \\
& \lambda_{*+}=\frac{1}{3(N+2)} \varepsilon, \quad g_{*+}=\frac{1}{3} \varepsilon, \quad \gamma_{*+}=\frac{1}{108} \varepsilon^{2},
\end{aligned}
$$

with the anomalous dimension at the fixed point obtained from (5.24). From (5.19) the eigenvalues of the stabilty matrix to lowest order are given by

$$
\kappa_{0, \pm}=\varepsilon, \quad \kappa_{1,-}=\frac{N-4}{3 N} \varepsilon, \quad \kappa_{1,+}=-\frac{1}{3} \varepsilon .
$$

For the second case the $\left(\phi^{2}\right)^{2}$ interaction is cancelled so this describes $N$ decoupled $\phi^{4}$ theories. For $N=2$ the two fixed points are equivalent and correspond to case a) in (3.9) with $\lambda_{0}=\lambda_{1}$ or $\lambda_{0}=0$. For $N=4$ the results in (5.42) corresponding to $x_{*}=x_{-}$are identical with (5.12). The higher order results for $\beta$-functions allow an extension of (5.43) as an expansion in $\varepsilon$. The results for $\kappa_{0,+}, \kappa_{1,+}$ match those for the Ising model as expected and as given in (3.12). To the next order

$$
\begin{aligned}
& \kappa_{0,-}=\varepsilon-\frac{(N-1)\left(17 N^{2}-4 N+212\right)}{27 N^{2}(N+2)} \varepsilon^{2}, \\
& \kappa_{1,-}=\frac{N-4}{3 N} \varepsilon-\frac{(N-1)\left(19 N^{3}-72 N^{2}-660 N+848\right)}{81 N^{3}(N+2)} \varepsilon^{2} .
\end{aligned}
$$

The critical point is stable for $\kappa_{1,-}>0$. Including three loop contributions this arises for exactly the same $N$ as in (5.30) when the $O(N)$ fixed point is unstable. In consequence the $O(N)$ theory with a hypercubic perturbation has a unique stable fixed point for all $N$.

Although the fixed point at $x_{*}=x_{-}$is stable for $N \gtrsim 4$ there may be slightly relevant $\phi^{4}$ perturbations. At this fixed point the anomalous dimensions to lowest order are determined by the eigenvalues of the operator

$$
\mathcal{D}=\varepsilon \frac{1}{3 N}\left(\frac{1}{2} \phi^{2} \partial^{2}+(\phi \cdot \partial)^{2}-\phi \cdot \partial+\frac{1}{2}(N-4) \sum_{i} \phi_{i}^{2} \partial_{i}^{2}\right)
$$

The $\frac{1}{24} N(N+1)(N+2)(N+3) \phi^{4}$ operators may be decomposed into irreducible repre- 
sentations of the hypercubic symmetry group to obtain

$$
\begin{aligned}
& \mathcal{D} \phi_{i} \phi_{j} \phi_{k} \phi_{l}=\varepsilon \frac{4}{N} \phi_{i} \phi_{j} \phi_{k} \phi_{l}, \quad i \neq j \neq k \neq l, \quad \operatorname{dim}\left(\begin{array}{c}
N \\
4
\end{array}\right) \\
& \mathcal{D} \phi_{i} \phi_{j}\left(\phi_{k} \phi_{k}-\phi_{l} \phi_{l}\right)=\varepsilon \frac{N+8}{3 N} \phi_{i} \phi_{j}\left(\phi_{k} \phi_{k}-\phi_{l} \phi_{l}\right), \quad i \neq j \neq k \neq l, \quad \operatorname{dim} \frac{1}{2} N(N-1)(N-3) \text {, } \\
& \mathcal{D} S_{i j}=\varepsilon \frac{2(N+2)}{3 N} S_{i j}, \quad \sum_{i} S_{i j}=0, \quad \operatorname{dim} \frac{1}{2} N(N-3), \\
& S_{i j}=\phi_{i}^{2} \phi_{j}^{2}-\frac{1}{N-2}\left(\left(\phi_{i}^{2}+\phi_{j}^{2}\right) \phi^{2}-\phi_{i}^{4}-\phi_{j}^{4}\right)+\frac{1}{(N-1)(N-2)}\left(\left(\phi^{2}\right)^{2}-\sum_{i} \phi_{i}{ }^{4}\right), i \neq j, \\
& \mathcal{D}\left(\phi_{i} \phi_{j}{ }^{3}-\phi_{i}{ }^{3} \phi_{j}\right)=\varepsilon\left(\phi_{i} \phi_{j}{ }^{3}-\phi_{i}{ }^{3} \phi_{j}\right), \quad i \neq j, \quad \operatorname{dim} \frac{1}{2} N(N-1), \\
& \mathcal{D}\left(\begin{array}{c}
\phi_{i} \phi_{j} \phi^{2} \\
\phi_{i} \phi_{j}{ }^{3}+\phi_{i}{ }^{3} \phi_{j}
\end{array}\right)=\varepsilon \frac{1}{3 N}\left(\begin{array}{cc}
2(N+6) & 2(N-4) \\
6 & 3 N
\end{array}\right)\left(\begin{array}{c}
\phi_{i} \phi_{j} \phi^{2} \\
\phi_{i} \phi_{j}{ }^{3}+\phi_{i}{ }^{3} \phi_{j}
\end{array}\right), i \neq j, \operatorname{dim} N(N-1), \\
& \mathcal{D}\left(\begin{array}{c}
\left(\phi_{i}{ }^{2}-\phi_{j}{ }^{2}\right) \phi^{2} \\
\phi_{i}{ }^{4}-\phi_{j}{ }^{4}
\end{array}\right)=\varepsilon \frac{1}{3 N}\left(\begin{array}{cr}
3 N+8 & 4(N-4) \\
6 & 6(N-2)
\end{array}\right)\left(\begin{array}{c}
\left(\phi_{i}{ }^{2}-\phi_{j}{ }^{2}\right) \phi^{2} \\
\phi_{i}{ }^{4}-\phi_{j}{ }^{4}
\end{array}\right), \quad i \neq j, \quad \operatorname{dim} 2(N-1) \\
& \mathcal{D}\left(\begin{array}{c}
\left(\phi^{2}\right)^{2} \\
\sum_{i} \phi_{i}^{4}
\end{array}\right)=\varepsilon \frac{2}{3 N}\left(\begin{array}{cc}
2(N+2) & 2(N-4) \\
3 & 3(N-2)
\end{array}\right)\left(\begin{array}{c}
\left(\phi^{2}\right)^{2} \\
\sum_{i} \phi_{i}^{4}
\end{array}\right), \quad \operatorname{dim} 2 .
\end{aligned}
$$

For any eigenvalue $\mu$ of $\mathcal{D}$ the associated eigenvector determines a scaling $\phi^{4}$ operator with scaling dimension $2(d-2)+\mu$ so that if $\kappa=\mu-\varepsilon<0$ it represents a relevant perturbation. For each operator in (5.46) the values of $\kappa / \varepsilon$ in turn are $\frac{4-N}{N}, \frac{2(4-N)}{3 N}, \frac{4-N}{3 N}, 0$, $\frac{1}{6 N}\left(12-N \pm \sqrt{N^{2}+24 N-48}\right), 0, \frac{3 N-4}{3 N}, 1, \frac{N-4}{3 N}$. The last two values correspond to the two perturbations which preserve hypercubic symmetry and just give (5.43). However other perturbations, which break the hypercubic symmetry, become relevant for $N>4$.

\subsubsection{Hypertetrahedral fixed points}

An additional case [39] is obtained by considering $N+1$ vectors $e_{i}^{\alpha}, \alpha=1,2, \ldots, N+1$, forming the vertices of a $N$-dimensional hypertetrahedron and satisfying, with a convenient normalisation,

$$
\sum_{\alpha} e_{i}^{\alpha}=0, \quad \sum_{\alpha} e_{i}^{\alpha} e_{j}^{\alpha}=\delta_{i j}, \quad e_{i}^{\alpha} e_{i}^{\beta}=\delta^{\alpha \beta}-\frac{1}{N+1} .
$$

We may then define

$$
d_{i j k l} \phi_{i} \phi_{j} \phi_{k} \phi_{l}=\sum_{\alpha}\left(e_{i}^{\alpha} \phi_{i}\right)^{4}-\frac{3 N}{(N+1)(N+2)}\left(\phi^{2}\right)^{2}
$$

In this case $-\frac{2(N-1)}{(N+1)(N+2)}\left(\phi^{2}\right)^{2} \leq d_{i j k l} \phi_{i} \phi_{j} \phi_{k} \phi_{l} \leq \frac{(N-1)(N-2)}{N(N+2)}\left(\phi^{2}\right)^{2}{ }^{5}$ The symmetry group is $H_{\text {tetrahedral }} \simeq A_{N} \otimes \mathbb{Z}_{2} \simeq \mathcal{S}_{N+1} \otimes \mathbb{Z}_{2}$, with $\mathcal{S}_{N+1}$ corresponding to the permutations of

\footnotetext{
${ }^{5}$ The bounds are equivalent to those for $\sum_{\alpha=1}^{N+1} x_{\alpha}{ }^{4} /\left(\sum_{\alpha=1}^{N+1} x_{\alpha}{ }^{2}\right)^{2}$ subject to $\sum_{\alpha=1}^{N+1} x_{\alpha}=0$. For $N$ even the lower bound is modified.
} 
$\underline{e}^{\alpha}$ or the vertices of the hypertetrahedron and $\mathbb{Z}_{2}$ to reflections $\phi_{i} \rightarrow-\phi_{i}$. This expression for $d_{i j k l}$ satisfies (5.2) with

$$
a=2 \frac{(N-1)(N-2)}{(N+1)(N+2)^{2}}, \quad b=\frac{N^{2}-3 N-2}{(N+1)(N+2)},
$$

and in (5.27)

$$
c=\frac{N^{6}-9 N^{5}+20 N^{4}+30 N^{3}-60 N^{2}-120 N-48}{(N+1)^{3}(N+2)^{3}} .
$$

For this case $a-2 \frac{N-1}{(N-2)^{2}} b^{2}=2 \frac{(N-1)(N-3)}{(N+1)^{2}(N-2)^{2}}$. The solutions of (5.4) give

$$
\mu_{+}=\frac{N-2}{N+2}, \quad d_{+}=N, \quad \mu_{-}=-\frac{2 N}{(N+1)(N+2)}, \quad d_{-}=\frac{1}{2}(N-2)(N+1) .
$$

For $N=2, d_{i j k l}=0$ and for $N=3,(5.48)$ reduces, up to an overall scale, to (5.38) which has cubic symmetry $\left(\mathcal{S}_{4} \subset \mathcal{S}_{3} \ltimes \mathbb{Z}_{2}^{3} \simeq \mathcal{S}_{4} \ltimes \mathbb{Z}_{2}\right.$, with $\mathcal{S}_{4}$ corresponding to permutations of the diagonals of the cube, in this case $b^{2} / a=\frac{1}{4}, c / b^{3}=-3$, for other $N$ the hypertetrahedral symmetry group is not a subgroup of the hypercubic group). For large $N a, b, c$ have the same limits as in the cubic case so the fixed points approach each other. However, unlike the cubic case, the hypertetrahedral theory contains an $H$ invariant cubic operator $\sum_{\alpha}\left(e_{i}^{\alpha} \phi_{i}\right)^{3}$.

The fixed points are determined by the roots of $f(x)=0$ in (5.13). Apart from $x=0$ these are $x_{-}=(N+2) / 2, x_{+}=(N-4)(N+1)(N+2) /(N-1)(N-2)$ and $f^{\prime}\left(x_{-}\right)=$ $-\frac{1}{2}(N-5)(N+2) /(N+1), f^{\prime}\left(x_{+}\right)=(N-5)(N-4)(N+2) /(N-1)(N-2)$. For $N>5$ $x_{*}=x_{-}$is then the stable fixed point. These roots are coincident for $N=5$. In (5.14)

$$
F(N)_{\text {tetrahedral }}=\frac{(N+2)^{2}(N-5)^{2}}{2(N-1)(N-2)(N+1)}<F(N)_{\text {cubic }} \text { for } N>3
$$

The couplings at the fixed points and the lowest order anomalous dimensions are then for $x_{*}=x_{-}$

$$
\lambda_{*-}=\frac{2(N+1)}{3(N+2)(N+3)} \varepsilon, \quad g_{*-}=\frac{N+1}{3(N+3)} \varepsilon, \quad \gamma_{*-}=\frac{(N+1)(N+7)}{108(N+3)^{2}} \varepsilon^{2},
$$

and for $x_{*}=x_{+}$

$$
\begin{aligned}
& \lambda_{*+}=\frac{(N-1)(N-2)}{3(N+2)\left(N^{2}-5 N+8\right)} \varepsilon, \quad g_{*+}=\frac{(N-4)(N+1)}{3\left(N^{2}-5 N+8\right)} \varepsilon, \\
& \gamma_{*+}=\frac{(N-1)(N-2)\left(N^{2}-6 N+11\right)}{108\left(N^{2}-5 N+8\right)^{2}} \varepsilon^{2} .
\end{aligned}
$$

In this case from (5.19)

$$
\kappa_{0, \pm}=\varepsilon, \quad \kappa_{1,-}=\frac{N-5}{3(N+3)} \varepsilon, \quad \kappa_{1,+}=-\frac{(N-4)(N-5)}{3\left(N^{2}-5 N+8\right)} \varepsilon .
$$

For the $a$-function

$$
A_{*-\text { tetrahedral }}-A_{*-\text { cubic }}=-\frac{(N-3)\left(N^{2}-3 N-6\right)}{54 N(N+3)^{2}},
$$


so that for $N \geq 5$ the tetrahedral fixed point has a lower $A$ and also a lower symmetry than the cubic one. The difference vanishes when $N=3$ since in this case the cubic and tetrahedral theories coincide. The marginal perturbations at this fixed point are analysed in appendix D.

The result (5.52) has a double zero at $N=5$ when (5.53) and (5.54) coincide. Taking into account higher order contributions for $N \approx 5$ the two fixed points have an expansion where

$$
\begin{aligned}
\lambda_{*+}-\lambda_{*-} & =a_{\lambda}(N, \varepsilon) \tilde{N}(N, \varepsilon)^{\frac{1}{2}}, \quad g_{*+}-g_{*-}=a_{g}(N, \varepsilon) \tilde{N}(N, \varepsilon)^{\frac{1}{2}}, \\
\tilde{N}(N, \varepsilon) & =\left(N-5-6 \varepsilon-\frac{281}{32} \varepsilon^{2}+\frac{61}{8} \zeta_{3} \varepsilon^{2}\right)^{2}-24 \varepsilon-\frac{289}{4} \varepsilon^{2}+30 \zeta_{3} \varepsilon^{2},
\end{aligned}
$$

where $a_{\lambda}(N, \varepsilon), a_{g}(N, \varepsilon)$ have no singularities as $N \rightarrow 5 . \tilde{N}(N, \varepsilon)=0$ determines $N_{\text {crit }}$ where new fixed points emerge. From (5.57), to $\mathrm{O}\left(\varepsilon^{2}\right)$,

$$
N_{\text {crit } \pm}=5+6 \varepsilon+\frac{281}{32} \varepsilon^{2}-\frac{61}{8} \zeta_{3} \varepsilon^{2} \pm \sqrt{24 \varepsilon+\frac{289}{4} \varepsilon^{2}-30 \zeta_{3} \varepsilon^{2}} .
$$

For $N_{\text {crit- }}<N<N_{\text {crit }}$ there are no fixed points.

It is natural to consider extensions of (5.47) based on regular polytopes for general $N$, generalisations of the Platonic solids, which define discrete subgroups $H$ of $\mathrm{SO}(N)$. However for $N \geq 5$ we have, apart from hypertetrahedrons or $N$-simplices, just hypercubes, with $2^{N}$ vertices $( \pm 1, \pm 1, \ldots, \pm 1)$, and hyperoctahedrons with $2 N$ vertices $(0,0, \ldots, \pm 1, \ldots, 0)$. These are dual by interchanging faces and vertices and have the same symmetry. In either case extending (5.48) is equivalent to (5.38). More generally vertex transitive or isogonal polytopes also define discrete subgroups of $\mathrm{SO}(N)$. An example are demihypercubes with $2^{N-1}$ vertices $( \pm 1, \pm 1, \ldots, \pm 1)$, with an even number of -1 's. For $N=3$ this is just a tetrahedron. For general $N$ the symmetry group is $D_{N} \simeq \mathcal{S}_{N} \ltimes \mathbb{Z}_{2}^{N} / \mathbb{Z}_{2}$. However for quartic polynomials $\sum_{\alpha}\left(e_{i}^{\alpha} \phi_{i}\right)^{4}=-2^{N} \sum_{i} \phi_{i}^{4}+3 \times 2^{N-1}\left(\phi^{2}\right)^{2}$ except when $N=4$ and there is an additional term $192 \phi_{1} \phi_{2} \phi_{3} \phi_{4}$ which is invariant under $D_{4}$. Nevertheless this is equivalent to the hypercubic case for all $N$, for $N=4, \sum_{\alpha}\left(e_{i}^{\alpha} \phi_{i}\right)^{4}=32 \sum_{i} \varphi_{i}^{4}$ taking $\varphi_{1}=\frac{1}{2}\left(\phi_{1}+\right.$ $\left.\phi_{2}+\phi_{3}+\phi_{4}\right), \varphi_{2}=\frac{1}{2}\left(\phi_{1}-\phi_{2}-\phi_{3}+\phi_{4}\right), \varphi_{3}=\frac{1}{2}\left(\phi_{1}-\phi_{2}+\phi_{3}-\phi_{4}\right), \varphi_{4}=\frac{1}{2}\left(\phi_{1}+\phi_{2}-\phi_{3}-\phi_{4}\right)$.

\subsection{Fixed points with continuous $H \subset O(N)$}

Theories in which the RG flow involves just two couplings can also be obtained by extending (5.2) to ${ }^{6}$

$$
d_{i j m n} d_{k l m n}=\frac{1}{N-1} a\left(\frac{1}{2} N\left(\delta_{i k} \delta_{j l}+\delta_{i l} \delta_{j k}\right)-\delta_{i j} \delta_{k l}\right)+e^{u} w_{u, i j k l}+b d_{i j k l},
$$

assuming $w_{u, i j k l}$ are a basis of $H$-invariant tensors satisfying ${ }^{7}$

$$
w_{u, i j k l}=w_{u, j i k l}=w_{u, k l i j}, \quad w_{u, i(j k l)}=0, \quad w_{u, i i k l}=0 .
$$

\footnotetext{
${ }^{6}$ For the RG flow for the theory defined by $(5.1)$ to be closed at one loop it is necessary to impose $d_{i j m n} d_{k l m n}+d_{i k m n} d_{j l m n}+d_{i l m n} d_{j k m n}=a\left(\delta_{i j} \delta_{k l}+\delta_{i k} \delta_{j l}+\delta_{i l} \delta_{j k}\right)+3 b d_{i j k l}$. In that case we may define $e^{u} w_{u, i j k l}=\frac{1}{3}\left(2 d_{i j m n} d_{k l m n}-d_{i k m n} d_{j l m n}-d_{i l m n} d_{j k m n}\right)+\frac{N+2}{6(N-1)} a\left(2 \delta_{i j} \delta_{k l}-\delta_{i k} \delta_{j l}-\delta_{i l} \delta_{j k}\right)$.

${ }^{7}$ If $c_{i k j l}=w_{i j k l}-w_{k j i l}$ then $c_{i k j l}=-c_{k i j l}=c_{j l i k}$ and $c_{i[k j l]}=0, c_{i j j l}=0$ and so has the symmetries of the Weyl tensor. $w_{i j k l}=\left(c_{i k j l}+c_{j k i l}\right) / 3$.
} 
Such tensors may be present for $N \geq 4$. For calculations beyond one loop we require

$$
\begin{aligned}
d_{i j m n} w_{u, k l m n} & =w_{u, i j m n} d_{k l m n}=f_{u}^{v} w_{v, i j k l}+h_{u} d_{i j k l}, \\
w_{u, i j m n} w_{v, k l m n} & =w_{v, i j m n} w_{u, k l m n} \\
& =\frac{1}{N-1} a_{u v}^{\prime}\left(\frac{1}{2} N\left(\delta_{i k} \delta_{j l}+\delta_{i l} \delta_{j k}\right)-\delta_{i j} \delta_{k l}\right)+e_{u v}^{\prime} w_{w, i j k l}+b_{u v}^{\prime} d_{i j k l} .
\end{aligned}
$$

There are various consistency conditions such as

$$
e^{u} a_{u v}^{\prime}=h_{v} a, \quad f_{u}^{v} a_{v w}^{\prime}=b_{u w}^{\prime} a, \quad f_{u}^{v} h_{v}=b_{u v}^{\prime} e^{v}, \quad e_{u v}^{\prime} a_{x w}^{\prime}=e_{u w}^{\prime x} a_{x v}^{\prime} .
$$

The eigenvalue equation (5.4) is extended to

$$
d_{i j k l} v_{k l}=\mu v_{i j}, \quad w_{u, i j k l} v_{k l}=\mu_{u} v_{i j}
$$

requiring

$\mu^{2}=e^{u} \mu_{u}+b \mu+\frac{N}{N-1} a, \quad \mu \mu_{u}=f_{u}^{v} \mu_{v}+h_{u} \mu, \quad \mu_{u} \mu_{v}=e_{u v}^{\prime}{ }^{w} \mu_{w}+b_{u v}^{\prime} \mu+\frac{N}{N-1} a_{u v}^{\prime}$.

Assuming (5.59) with (5.60) the one loop $\beta$-functions remain as in (5.9). At two loops in (5.23), using $w_{u, i m j n} d_{k l m n}=-\frac{1}{2} w_{u, i j m n} d_{k l m n}$, and (5.37)

$$
\Delta \beta_{g}^{(2)}=3 e^{u} h_{u} g^{3}, \quad \Delta \beta_{\rho, i j}{ }^{(2)}=\frac{1}{2} g^{2} e^{u} w_{u, i j k l} \rho_{k l} .
$$

and, since in (5.27) $\Delta \mathcal{A}=-a e^{u} h_{u}$, at three loops (5.28) is modified by

$$
\begin{aligned}
\Delta \beta_{\lambda}^{(3)} & =-a e^{u} h_{u}\left(\frac{11}{2}+3 \zeta_{3}\right) g^{4} \\
\Delta \beta_{g}^{(3)} & =-\frac{3}{4}\left(29 b e^{u} h_{u}-8 e^{u} f_{u}^{v} h_{v}\right) g^{4}-6 e^{u} h_{u}\left(7+12 \zeta_{3}\right) \lambda g^{3} \\
\Delta \beta_{\rho, i j}{ }^{(3)} & =-\frac{11}{4} e^{u} h_{u} g^{3} d_{i j k l} \rho_{k l}-\left(3 g^{2} \lambda+\frac{5}{4} b g^{3}\right) e^{u} w_{u, i j k l} \rho_{k l}+g^{3} e^{u} f_{u}^{v} w_{v, i j k l} \rho_{k l} .
\end{aligned}
$$

As a consequence of the changes in the $\beta$-functions in (5.65) and (5.66) then in (5.35) there are additional terms

$$
\begin{aligned}
\Delta F_{1}(N)= & -\frac{6(N-4)}{N+2} \frac{e^{u} h_{u}}{a} \\
\Delta F_{2}(N)= & -\frac{3\left(N^{3}+35 N^{2}-82 N-8\right)}{4(N-1)(N+2)^{2}} \frac{e^{u} h_{u}}{a}+\frac{9(N-4)^{2}(N+8)}{4(N+2)^{4}}\left(\frac{e^{u} h_{u}}{a}\right)^{2} \\
& -\frac{16(N-4)^{3}}{9(N+2)^{2}} \frac{e^{u} f_{u}^{v} h_{v}}{b^{3}}-\frac{3(N-4)(N-28)}{(N+2)^{2}} \frac{e^{u} h_{u}}{a} \zeta_{3} .
\end{aligned}
$$

A particular example arises when $d_{i j k l}$ is the symmetric traceless rank 4 tensor for a Lie group when there is an independent quartic Casimir. For a simple Lie algebra with a basis $\left\{t_{i}\right\}$ where we assume, with $i, j, \cdots=1, \ldots, N$,

$$
\left[t_{i}, t_{j}\right]=f_{i j k} t_{k}, \quad f_{i k l} f_{j k l}=C \delta_{i j}, \quad \operatorname{tr}\left(t_{i} t_{j}\right)=-R \delta_{i j}, \quad t_{i} t_{i}=-C_{R} \mathbb{1},
$$


then we may take

$$
d_{i j k l} \phi_{i} \phi_{j} \phi_{l} \phi_{l}=\operatorname{tr}\left(t_{\phi}^{4}\right)+y\left(\phi^{2}\right)^{2}, \quad t_{\phi}=t_{i} \phi_{i}
$$

with $y$ determined to ensure $d_{i j k l}$ is traceless. Using $t_{i} t_{\phi} t_{i}=\left(\frac{1}{2} C-C_{R}\right) t_{\phi}$ this gives

$$
y=-\frac{3 C_{R}-\frac{1}{2} C}{N+2} R
$$

Similarly we may define, if $\operatorname{tr}\left(t_{i}\left\{t_{j}, t_{k}\right\}\right)=0$,

$$
w_{i j k l} \phi_{k} \phi_{l}=-\operatorname{tr}\left(\left[t_{i}, t_{\phi}\right]\left[t_{j}, t_{\phi}\right]\right)+z\left(\delta_{i j} \phi^{2}-\phi_{i} \phi_{j}\right), \quad z=-\frac{C R}{N-1} .
$$

For the case of $O(n)$ then $t_{i}$ are a basis of $n \times n$ antisymmetric matrices so that the theory reduces to antisymmetric tensor fields $\varphi_{a b}=\left(t_{\phi}\right)_{a b}, a, b=1, \ldots, n$, as discussed in $[40,41]$. In this case in $(5.68)$

$$
C=2(n-2), \quad R=2, \quad C_{R}=n-1, \quad N=\frac{1}{2} n(n-1),
$$

so that $y=-2(2 n-1) /(N+2), z=-8 /(n+1)$. In this case for $d_{i j k l} \phi_{i} \phi_{j} \phi_{l} \phi_{l}$ given in $(5.69)-\frac{2\left(n^{2}-4\right)}{n(N+2)}\left(\phi^{2}\right)^{2} \leq d_{i j k l} \phi_{i} \phi_{j} \phi_{k} \phi_{l} \leq \frac{(n-2)(n-3)}{N+2}\left(\phi^{2}\right)^{2}$ (for $n$ odd the lower bound is modified). With the completeness relation $t_{i} \operatorname{tr}\left(t_{i} Y\right)=-R Y$ for any $Y^{T}=-Y$ and also $t_{i} M t_{i}=\left(C_{R}-\frac{1}{2} C\right) M^{T}-\mathbb{1} \operatorname{tr}(M)$. For this case the definition (5.69) gives $d_{i j k l}=0$ for $n=2,3$ and with the above results we may verify (5.59) with

$$
a=\frac{2(n-2)(n-3)(n+1)(n+2)}{3(N+2)^{2}}, \quad b=\frac{(n-5)(n+4)(2 n-1)}{9(N+2)}, \quad e=\frac{2}{27}(n+1),
$$

and, with more complexity,

$$
c=\frac{n^{8}-84 n^{7}+302 n^{6}+2212 n^{5}-6215 n^{4}-13944 n^{3}+27456 n^{2}-40448 n+15616}{216(N+2)^{3}} .
$$

With $a, b$ given by (5.73) then in (5.14) $F(N)<0$ for $n>\frac{1}{4}(2+3 \sqrt{22})$ so that there are only fixed points for real non zero couplings $\lambda, g$ when $n=4$. In (5.61)

$$
\begin{array}{lll}
h=\frac{8(N+2)}{3(n+1)}, & f=\frac{(n-8)(n+1)^{2}}{9(N+2)}, \\
a^{\prime}=\frac{24(n-2)(n-3)(n+2)}{(n+1)(N+2)}, & b^{\prime}=4(n-8), & e^{\prime}=\frac{4(N+11)}{3(n+1)},
\end{array}
$$

which in conjunction with (5.74) determine higher loop contributions to $\beta$-functions. ${ }^{8}$

\footnotetext{
${ }^{8}$ The results to three loops are in numerical agreement with [41] in terms of couplings $g_{1}=6 \lambda+2 y g, g_{2}=8 g$.
} 


\subsection{1 $O(m) \times O(n)$ fixed points}

A theory with non trivial fixed points is obtained by considering a symmetry breaking where $O(N) \rightarrow O(m) \times O(n) / \mathbb{Z}_{2}, N=m n$ as was considered in [30,42]. This case can be discussed in the present context by taking $\phi_{i}=\Phi_{a r}$ with $a=1, \ldots, m, r=1, \ldots, n$ and, with the usual summation convention for repeated paired indices,

$$
d_{i j k l} \phi_{i} \phi_{j} \phi_{k} \phi_{l}=\Phi_{a r} \Phi_{b r} \Phi_{a s} \Phi_{b s}-\frac{m+n+1}{N+2}\left(\Phi^{2}\right)^{2} .
$$

This vanishes for $m=1$ or $n=1$ and for $m \leq n$, satisfies the bounds $-\frac{(m-1)(m+2)}{m(N+2)}\left(\Phi^{2}\right)^{2} \leq$ $d_{i j k l} \phi_{i} \phi_{j} \phi_{k} \phi_{l} \leq \frac{(m-1)(n-1)}{N+2}\left(\Phi^{2}\right)^{2}$. In this case there are two tensors $w_{u, i j k l}, u=1,2$, which are determined by

$$
\begin{aligned}
& w_{1, i j k l} \phi_{k} \phi_{l}=\delta_{r s} \Phi_{a t} \Phi_{b t}-\delta_{a b} \Phi_{c r} \Phi_{c s}-\frac{n-m}{N-1}\left(\delta_{a b} \delta_{r s} \Phi^{2}-\Phi_{a r} \Phi_{b s}\right) \\
& w_{2, i j k l} \phi_{k} \phi_{l}=\delta_{r s} \Phi_{a t} \Phi_{b t}+\delta_{a b} \Phi_{c r} \Phi_{c s}-2 \Phi_{a s} \Phi_{b r}-\frac{m+n-2}{N-1}\left(\delta_{a b} \delta_{r s} \Phi^{2}-\Phi_{a r} \Phi_{b s}\right) .
\end{aligned}
$$

In $(5.2)$

$$
\begin{aligned}
a & =\frac{(m-1)(m+2)(n-1)(n+2)}{3(N+2)^{2}}, & & \\
b & =\frac{D_{m n}}{9(N+2)}, & D_{m n} & =m n(m+n)+4 m n-10(m+n)-4, \\
e^{1} & =\frac{1}{18}(n-m), & e^{2} & =\frac{1}{54}(m+n+4),
\end{aligned}
$$

and in (5.27)

$$
\begin{aligned}
c= & \frac{1}{216(m n+2)^{3}}(m n-4(m+n)-2) \\
& \times\left(5 m^{3} n^{3}+10 m^{2} n^{2}(m+n)-40 m n\left(m^{2}+n^{2}\right)+20 m^{2} n^{2}-88 m n(m+n)\right. \\
& \left.\quad+16\left(m^{2}+n^{2}\right)+284 m n-24(m+n)+240\right),
\end{aligned}
$$

and in (5.60)

$$
\begin{array}{rlrl}
f_{1}^{1} & =\frac{1}{6}(n+m+2)-\frac{2(m+n+1)}{3(N+2)}, & f_{1}^{2}=\frac{1}{18}(n-m), \\
f_{2}^{1}=\frac{1}{6}(n-m), & f_{2}^{2}=\frac{1}{18}(n+m-2)-\frac{2(m+n+1)}{3(N+2)}, \\
h_{1}=(n-m)\left(\frac{1}{3}+\frac{1}{N-1}\right), & h_{2}=(n+m-2)\left(\frac{1}{3}+\frac{1}{N-1}\right), \\
a_{11}^{\prime}=\frac{(m-1)(n-1)(2 N+3 m+3 n+4)}{(N-1)(N+2)}, & a_{12}^{\prime}=a_{21}^{\prime}=3 \frac{(m-1)(n-1)(n-m)}{(N-1)(N+2)}, \\
a_{22}^{\prime}=3 \frac{(m-1)(n-1)(2 N+m+n-4)}{(N-1)(N+2)}, & \\
b_{11}^{\prime}=n+m, \quad b_{12}^{\prime}=b_{21}^{\prime}=n-m, & b_{22}^{\prime}=m+n-8 .
\end{array}
$$


The coefficients $e_{u v}^{\prime}{ }^{w}$ are also determined but not quoted here. For $m=n$ only the $w_{2}$ tensor is relevant.

These results suffice to determine the $\beta$-functions to three loops. ${ }^{9}$ Solving the eigenvalue equations (5.64) for the $O(m) \times O(n)$ case gives four possibilities for $\left(\mu, \mu_{1}, \mu_{2}\right)$

(i) $\left(\frac{(m-1)(m+2) n}{3(N+2)},-\frac{\left(m^{2}-1\right) n}{N-1}, \frac{(m-1)^{2} n}{N-1}\right), \quad \operatorname{dim}=\frac{1}{2}(n-1)(n+2)$,

(ii) $\left(\frac{m(n-1)(n+2)}{3(N+2)}, \frac{m\left(n^{2}-1\right)}{N-1}, \frac{m(n-1)^{2}}{N-1}\right), \quad \operatorname{dim}=\frac{1}{2}(m-1)(m+2)$,

(iii) $\left(\frac{N-2 m-2 n}{3(N+2)}, \frac{n-m}{N-1},-\frac{2 N-m-n}{N-1}\right), \quad \operatorname{dim}=\frac{1}{4}(m-1)(m+2)(n-1)(n+2)$,

(iv) $\left(-\frac{(m+2)(n+2)}{3(N+2)}, \frac{n-m}{N-1}, \frac{2 N+m+n-4}{N-1}\right), \quad \operatorname{dim}=\frac{1}{4} m(m-1) n(n-1)$,

corresponding to the decomposition of the eigenvectors $v_{a r, b s}$ into irreducible representations of $O(m) \times O(n), v_{a r, a s}, v_{a r, b r}, v_{a r, b s}+v_{b r, a s}-\frac{2}{m} \delta_{a b} v_{c r, c s}-\frac{2}{n} \delta_{r s} v_{a t, b t}$ and $v_{a r, b s}-v_{b r, a s}$. In (5.14)

$$
F(m, n)_{O(m) \times O(n)}=\frac{1}{9 a} R_{m n}, \quad R_{m n}=m^{2}+n^{2}-10 m n-4(m+n)+52,
$$

with notation borrowed from [42]. Positivity of $F$ then requires $R_{m n}>0$ so that $n$ is restricted by $n<5 m+2-2 \sqrt{6(m-1)(m+2)}$ or $n>5 m+2+2 \sqrt{6(m-1)(m+2)}$. This excludes $m=n$ except when $m=n=2$. With the results in (5.67) these boundaries are modified in the $\varepsilon$ expansion to

$$
\begin{aligned}
& n_{\text {crit } \pm}=5 m+2 \pm 2 \sqrt{6(m-1)(m+2)}-(5 m+2) \varepsilon \mp \frac{25 m^{2}+22 m-32}{2 \sqrt{6(m-1)(m+2)}} \varepsilon \\
& +\left(\frac{8 m^{5}+31 m^{4}-426 m^{3}-1376 m^{2}+1184 m+1632}{8(m-7)(m+8)(m-1)(m+2)} \pm \frac{20 m^{4}+73 m^{3}-1230 m^{2}-2960 m+6176}{8(m-7)(m+8) \sqrt{6(m-1)(m+2)}}\right) \zeta_{3} \varepsilon^{2} \\
& +\frac{4 m^{7}+21 m^{6}-417 m^{5}-1883 m^{4}+9288 m^{3}+45444 m^{2}+18880 m-33024}{24(m-7)^{2}(m+8)^{2}(m-1)(m+2)} \varepsilon^{2} \\
& \pm \frac{5 m^{8}+58 m^{7}-389 m^{6}-5854 m^{5}+7756 m^{4}+139240 m^{3}+176192 m^{2}-160256 m-253292}{32(m-7)^{2}(m+8)^{2}(m-1)(m+2) \sqrt{6(m-1)(m+2)}} \varepsilon^{2}
\end{aligned}
$$

To $\mathrm{O}(\varepsilon)$ this agrees with [42] and to $\mathrm{O}\left(\varepsilon^{2}\right)$ when $m=2$ with [43] and for any $m$ with [30]. For $n>n_{\text {crit+ }}$ and $n<n_{\text {crit- }}$ there are two fixed points with non zero $g$. The $O(N)$ fixed point becomes unstable when $n>N_{\text {crit }, H} / m$ as given in (5.30).

At the fixed point to lowest order

$$
\lambda_{* \pm}=\frac{1}{2(N+2)} \frac{D_{m n} \pm(N+2) \sqrt{R_{m n}}}{D_{m n} \pm 6 \sqrt{R_{m n}}} \varepsilon, \quad g_{* \pm}=\frac{3(N-4)}{D_{m n} \pm 6 \sqrt{R_{m n}}} \varepsilon,
$$

and from (5.19) the stability matrix eigenvalues are

$$
\kappa_{0, \pm}=\varepsilon, \quad \kappa_{1, \pm}= \pm \frac{(N-4) \sqrt{R_{m n}}}{D_{m n} \pm 6 \sqrt{R_{m n}}} \varepsilon .
$$

${ }^{9}$ The $\beta$-functions to three loops obtained from $(5.9),(5.23),(5.28)$ in conjunction with $(5.65),(5.66)$ and $(5.78),(5.79),(5.80)$ are identical with the results of Pelissetto et al. [30] where their results are expressed in terms of the couplings $u=6 \lambda+2(1-(m+n+1) /(m n+2)) g, v=2 g$. 
From (5.25)

$$
A_{* \pm}=\frac{(N-4)^{2} R_{m n}}{48\left(D_{m n} \pm 6 \sqrt{R_{m n}}\right)^{2}} N \varepsilon^{3}-\frac{1}{48} N \varepsilon^{3} .
$$

For a fixed point $R_{m n}>0$ and so this respects the bound (5.20). In application to condensed matter systems the two fixed points, denoted by,+- , are referred to as chiral and anti-chiral [42]. Since $A_{*+}<A_{*-}$ the chiral fixed point is therefore the stable one.

At higher order the results are complicated but for $n \gg m$ and to order $\varepsilon^{3}$, taking into account two and three loop contributions,

$$
\begin{aligned}
& \lambda_{* \pm} \sim\left\{\begin{array}{l}
\frac{1}{N}\left(1-\frac{2}{N}\right) \varepsilon+\frac{3}{2 N n}(m+1)\left(-2 \varepsilon+3 \varepsilon^{2}-\frac{11}{8} \varepsilon^{3}\right) \\
\frac{1}{N^{2}}(m-1)(m+2)\left(3 \varepsilon-2 \varepsilon^{2}-\frac{3}{2} \varepsilon^{3}\right)
\end{array}\right. \\
& g_{* \pm} \sim\left\{\begin{array}{l}
\frac{3}{n}\left(\varepsilon+\frac{1}{n}\left(-(m+4) \varepsilon+\frac{3}{2}(m+3) \varepsilon^{2}-\frac{1}{16}(13 m+33) \varepsilon^{3}\right)\right) \\
\frac{3}{n}(\varepsilon \\
\left.\quad+\frac{1}{N}\left(-(m-2)(m+6) \varepsilon+\frac{1}{2}\left(3 m^{2}+9 m-34\right) \varepsilon^{2}-\frac{1}{16}\left(13 m^{2}+33 m-162\right) \varepsilon^{3}\right)\right)
\end{array}\right.
\end{aligned}
$$

Correspondingly in this limit, with notation from (4.45),

$$
\gamma_{ \pm} \sim k_{ \pm} \frac{1}{4 n}\left(\varepsilon^{2}-\frac{1}{4} \varepsilon^{3}\right), \quad \gamma_{\sigma \pm} \sim\left\{\begin{array}{l}
\varepsilon-k_{+} \frac{2}{n}\left(3 \varepsilon-\frac{13}{4} \varepsilon^{2}+\frac{3}{16} \varepsilon^{3}\right) \\
k_{-} \frac{2}{n}\left(3 \varepsilon-\frac{13}{4} \varepsilon^{2}+\frac{3}{16} \varepsilon^{3}\right)
\end{array}\right.
$$

There are also similar results for $\gamma_{\rho}$ which decomposes into four cases depending on the eigenvalues in (5.81)

(i) $\gamma_{\rho \pm} \sim 2 \gamma_{ \pm}+\frac{2}{n} k_{ \pm}\left(-\varepsilon+\frac{1}{2} \varepsilon^{2}+\frac{1}{4} \varepsilon^{3}\right)$,

(ii) $\gamma_{\rho \pm} \sim \varepsilon+\left\{\begin{array}{l}\frac{1}{n}\left(-(m+3) \varepsilon+\frac{1}{4}(5 m+13) \varepsilon^{2}-\frac{3}{16}(m+1) \varepsilon^{3}\right) \\ \frac{1}{N}\left(-(m-2)(m+5) \varepsilon-\frac{1}{4}\left(5 m^{2}+13 m-50\right) \varepsilon^{2}-\frac{1}{16}\left(3 m^{2}+3 m-22\right) \varepsilon^{3}\right)\end{array}\right.$

(iii) $\gamma_{\rho \pm} \sim 2 \gamma_{ \pm}+\left\{\begin{array}{l}\frac{1}{n}\left(\varepsilon-\frac{1}{2} \varepsilon^{2}-\frac{1}{4} \varepsilon^{3}\right) \\ \frac{1}{N}(m-2)\left(\varepsilon-\frac{1}{2} \varepsilon^{2}-\frac{1}{4} \varepsilon^{3}\right)\end{array}\right.$

(iv) $\gamma_{\rho \pm} \sim 2 \gamma_{ \pm}+\left\{\begin{array}{l}\frac{1}{n}\left(-\varepsilon+\frac{1}{2} \varepsilon^{2}+\frac{1}{4} \varepsilon^{3}\right) \\ \frac{1}{N}(m+2)\left(-\varepsilon+\frac{1}{2} \varepsilon^{2}+\frac{1}{4} \varepsilon^{3}\right)\end{array}\right.$

At the fixed point $\Delta_{\sigma, \rho}=d-2+\gamma_{\sigma, \rho *}$. For case (ii) $\Delta_{\rho * \pm}, \Delta_{\sigma *+}=2+\mathrm{O}(1 / n)$, the large $n$ analysis is based on couplings $\rho_{a b} \Phi_{a r} \Phi_{b r}$, with $\rho_{a b}$ symmetric and traceless, and $\sigma \Phi^{2}$ which have dimension $d$, for the anti-chiral fixed point only the $\rho_{a b}$ coupling is relevant. The results in (5.89) show the universal behaviour at large $n$ expected by large $n$ results [30-32]. 


\subsection{2 $M N$ fixed points}

An alternative breaking of $O(N)$ symmetry (referred to in some literature as the $M N$ model [9]) is realised when $O(N) \rightarrow \mathcal{S}_{n} \ltimes O(m)^{n}$ for $N=m n, \phi_{i}$ is decomposed as $n$ $m$-vectors $\vec{\varphi}_{r}$ and

$$
d_{i j k l} \phi_{i} \phi_{j} \phi_{k} \phi_{l}=\sum_{r}\left(\vec{\varphi}_{r}^{2}\right)^{2}-\frac{m+2}{N+2}\left(\vec{\varphi}^{2}\right)^{2}, \quad \vec{\varphi}^{2}=\sum_{r} \vec{\varphi}_{r}^{2},
$$

which satisfies $-\frac{2(n-1)}{n(N+2)}\left(\vec{\varphi}^{2}\right)^{2} \leq d_{i j k l} \phi_{i} \phi_{j} \phi_{k} \phi_{l} \leq \frac{(m-1) n}{N+2}\left(\vec{\varphi}^{2}\right)^{2}$. This expression reduces to the hypercubic model in (5.38) for $m=1$ and if in (5.1) $\lambda=\frac{m+2}{3(N+2)} g$ to $n$ decoupled $O(m)$ theories. Past discussions include [44-46]. The $\beta$-functions for such theories have been determined to four loops [47] for $m=2$ and are physically relevant for $m=2, n=2,3$. For $m=n=2$ this case with $O(2)^{2}$ symmetry is identical with the $O(2) \times O(2)$ symmetric theory, taking $\vec{\varphi}_{1}=\frac{1}{\sqrt{2}}\left(\Phi_{11}-\Phi_{22}, \Phi_{12}+\Phi_{21}\right), \vec{\varphi}_{2}=\frac{1}{\sqrt{2}}\left(\Phi_{11}+\Phi_{22}, \Phi_{12}-\Phi_{21}\right), \vec{\varphi}^{2}=\Phi^{2}$, $\sum_{r}\left(\vec{\varphi}_{r}^{2}\right)^{2}=-\Phi_{a r} \Phi_{b r} \Phi_{a s} \Phi_{b s}+\frac{3}{2}\left(\Phi^{2}\right)^{2}$. The $\beta$-functions in the two cases are related by

$$
\left.\beta_{\lambda}(\lambda, g)\right|_{O(2) \times O(2)}=\left.\beta_{\lambda}(\lambda,-g)\right|_{O(2)^{2}},\left.\quad \beta_{g}(\lambda, g)\right|_{O(2) \times O(2)}=-\left.\beta_{g}(\lambda,-g)\right|_{O(2)^{2}} .
$$

In the framework described here there is a single mixed symmetry tensor

$$
w_{i j k l} \phi_{i} \phi_{j} \phi_{k}^{\prime} \phi_{l}^{\prime} \rightarrow \sum_{r}\left(\vec{\varphi}_{r}^{2} \vec{\varphi}_{r}^{\prime 2}-\left(\vec{\varphi}_{r} \cdot \vec{\varphi}_{r}^{\prime}\right)^{2}\right)-\frac{m-1}{N-1}\left(\vec{\varphi}^{2} \vec{\varphi}^{\prime 2}-\left(\vec{\varphi} \cdot \vec{\varphi}^{\prime}\right)^{2}\right) \text {. }
$$

This gives

$$
\begin{aligned}
a=\frac{2 m(m+2)(n-1)}{3(N+2)^{2}}, & b=\frac{(n-1) m(m+8)+(m+2)(m-4)}{9(N+2)}, \quad e=\frac{2}{27}(m+2), \\
c=\frac{(m n-2 m-2)}{27(m n+2)^{3}}\left(5 m^{3} n^{2}+22 m^{2} n^{2}-10 m^{3} n-44 m^{2} n+4 m^{2}-12 m+8\right), & \\
f=\frac{2(N-1)(m+2)}{9(N+2)}, & h=\frac{(N+2)(m-1)}{3(N-1)}, \\
a^{\prime}=\frac{3(m-1) m(n-1)}{(N-1)(N+2)}, & b^{\prime}=m-1, \quad e^{\prime}=\frac{1}{3}(2 m-5)-2 \frac{m-1}{N-1} .
\end{aligned}
$$

For eigenvectors of the $d, w$ tensors with eigenvalues $\left(\mu, \mu^{\prime}\right)$ there are now three cases

$$
\begin{array}{lll}
\left(\frac{N(m+2)}{3(N+2)}, \frac{N(m-1)}{N-1}\right), & \operatorname{dim}=n-1, \\
\left(-\frac{2(m+2)}{3(N+2)}, \frac{m-1}{N-1}\right), & \operatorname{dim}=\frac{1}{2} m^{2} n(n-1), \\
\left(\frac{2 m(n-1)}{3(N+2)},-\frac{m(n-1)}{N-1}\right), & \operatorname{dim}=\frac{1}{2}(m-1)(m+2) n .
\end{array}
$$

The results in (5.93) are sufficient to determine the $\beta$-functions in this case to three loops for general $m, n .^{10}$

\footnotetext{
${ }^{10}$ The results are in accord with [47] for $m=2$ expressed in terms of couplings, up to a scale factor, $u=3 \lambda-(m+2) /(m n+2) g, v=g$. In general $\left.\beta_{u}\right|_{u=0}=0$ reflecting the decoupled fixed point.
} 
The polynomial $f(x)$, as defined in (5.13), now has roots, apart from $x=0$, given by $x_{-}=(N-4)(N+2) / 2 m(n-1), x_{+}=3(N+2) /(m+2), x_{+}>x_{-}$for $m<4$. In this case $f^{\prime}\left(x_{ \pm}\right)= \pm \frac{1}{3}(4-m) x_{ \pm}$. According to (5.19) a stable fixed point requires $f^{\prime}\left(x_{*}\right)<0$. For $N>4$, when the $O(N)$ symmetric fixed point has relevant perturbations, the stable fixed point is given by $x_{*}=x_{-}$or $x_{*}=x_{+}$depending on whether $m<4$ or $m>4$. In (5.14)

$$
F(m, n)_{O(m)^{n}}=\frac{(m-4)^{2}(N+2)}{6 m(m+2)(n-1)},
$$

so that for this breaking there are always non trivial additional fixed points which coincide when $m=4$. The two fixed points extending (5.42) are then given by

$$
\begin{array}{ll}
\lambda_{*-}=\frac{6(N-m)}{(N+2)((m+8) N-16(m-1))} \varepsilon, & g_{*-}=\frac{3(N-4)}{(m+8) N-16(m-1)} \varepsilon, \\
\gamma_{*-}=\frac{(N-m)(m(N+2)-10 m+16)}{4((m+8) N-16(m-1))^{2}} \varepsilon^{2}, & \\
\lambda_{*+}=\frac{m+2}{(m+8)(N+2)} \varepsilon, \quad g_{*+}=\frac{3}{m+8} \varepsilon, \quad \gamma_{*+}=\frac{m+2}{4(m+8)^{2}} \varepsilon^{2} .
\end{array}
$$

The stability matrix eigenvalues at lowest order are then

$$
\kappa_{0, \pm}=\varepsilon, \quad \kappa_{1,+}=\frac{m-4}{m+8} \varepsilon, \quad \kappa_{1,-}=-\frac{(m-4)(N-4)}{(m+8) N-16(m-1)} \varepsilon
$$

The fixed point realised for $x_{*}=x_{+}$corresponds to decoupled $O(m)$ theories, this is the stable fixed point for $m>4$. Using (5.8)

$$
\begin{aligned}
& A_{*+}=\frac{(m-4)^{2}}{48(m+8)^{2}} N \varepsilon^{3}-\frac{1}{48} N \varepsilon^{3}, \\
& A_{*-}=\frac{(m-4)^{2}(N-4)^{2}}{48((m+8) N-16(m-1))^{2}} N \varepsilon^{3}-\frac{1}{48} N \varepsilon^{3} .
\end{aligned}
$$

For a positive potential at the $x_{*}=x_{-}$fixed point we should require $(m+8) N>$ $16(m-1)$ and then stability requires $m<4$ or $m=1,2,3$.

\section{Scalar theories in $3-\varepsilon$ dimensions}

An $\varepsilon$ expansion starting from three dimensional renormalisable theories determines the properties of tricritical (since there are at least two relevant operators if $\mathbb{Z}_{2}$ symmetry is imposed for a single component field) fixed points [48, 49]. Such $\phi^{6}$ theories have been considered from a CFT perspective in [50, 51].

For our discussion we assume $N$-component scalar fields $\phi_{i}$ and initially a general sextic potential $V(\phi)=\frac{1}{6 !} \lambda_{i k l m n p} \phi_{i} \phi_{j} \phi_{k} \phi_{l} \phi_{m} \phi_{n}+\ldots$ After rescaling $V \rightarrow(8 \pi)^{2} V$ the lowest order, two loop, result for the $\beta$-functions for the couplings contained in $V$ is determined by

$$
\beta_{V}(\phi)=\varepsilon\left(V(\phi)-\frac{1}{2} \phi_{i} V_{i}(\phi)\right)+\frac{1}{3} V_{i j k}(\phi) V_{i j k}(\phi),
$$


with $V_{i}(\phi)=\partial_{i} V(\phi)$. For the next order, at four loops, ${ }^{11}$

$$
\begin{aligned}
\beta_{V}(\phi)^{(4)}= & \frac{1}{6} V_{i j}(\phi) V_{i k l m n}(\phi) V_{j k l m n}(\phi)-\frac{4}{3} V_{i j k}(\phi) V_{i l m n}(\phi) V_{j k l m n}(\phi) \\
& -\frac{\pi^{2}}{12} V_{i j k l}(\phi) V_{k l m n}(\phi) V_{i j m n}(\phi)+\phi_{i} \gamma_{i j}{ }^{(4)} V_{j}(\phi),
\end{aligned}
$$

with the four loop anomalous dimension matrix

$$
\gamma_{i j}^{(4)}=\frac{1}{90} \lambda_{i k l m n p} \lambda_{j k l m n p} .
$$

For the $O(N)$ symmetric case the potential is restricted to the form

$$
V(\phi)=\frac{\lambda}{48}\left(\phi^{2}\right)^{3}
$$

From (6.1),

$$
\beta_{\lambda}=-2 \varepsilon \lambda+4(3 N+22) \lambda^{2}
$$

and from $(6.3)$

$$
\gamma_{i j}=\gamma_{\phi} \delta_{i j}, \quad \gamma_{\phi}^{(4)}=\frac{1}{6}(N+2)(N+4) \lambda^{2} .
$$

The four loop result gives

$$
\beta_{\lambda}{ }^{(4)}=-4\left(53 N^{2}+858 N+3304\right) \lambda^{3}-\frac{1}{2} \pi^{2}\left(N^{3}+34 N^{2}+620 N+2720\right) \lambda^{3} .
$$

Equivalent results were obtained in [53] and extended to six loops in $[54,55]$. At the fixed point to lowest order

$$
\lambda_{*}=\frac{\varepsilon}{2(3 N+22)}, \quad \gamma_{\phi *}=\frac{(N+2)(N+4)}{24(3 N+22)^{2}} \varepsilon^{2} .
$$

For large $N$ the perturbative contributions to the $\beta$-function and the anomalous dimension $\gamma_{\phi}$ at higher orders have a leading $N$ dependence of the form $(N \lambda)^{p} N^{\left\lfloor\frac{1}{2}(p-3)\right\rfloor}$ and $(N \lambda)^{p} N^{\left\lfloor\frac{1}{2}(p-2)\right\rfloor}, p=2,3, \ldots$, respectively. ${ }^{12}$ Hence with a rescaled coupling

$$
\beta_{\tilde{\lambda}}=-2 \varepsilon \tilde{\lambda}+\frac{1}{N}\left(12 \tilde{\lambda}^{2}-\frac{1}{2} \pi^{2} \tilde{\lambda}^{3}\right)+\mathrm{O}\left(\frac{1}{N^{2}}\right), \quad \tilde{\lambda}=N^{2} \lambda
$$

A large $N$ IR fixed point is given, at leading order, by

$$
N \varepsilon=6 \tilde{\lambda}_{*}-\frac{1}{4} \pi^{2} \tilde{\lambda}_{*}^{2} \leq \frac{36}{\pi^{2}} \Rightarrow \tilde{\lambda}_{*}=\frac{4}{\pi^{2}}\left(3-\sqrt{9-\pi^{2} N \varepsilon / 4}\right) \approx \frac{1}{6} N \varepsilon+\frac{\pi^{2}}{864}(N \varepsilon)^{2} .
$$

The bound [53] determines the radius of convergence of the $\varepsilon$-expansion. At the fixed point

$$
\beta_{\tilde{\lambda}^{\prime}}\left(\tilde{\lambda}_{*}\right)=4 \varepsilon-\frac{12}{N} \tilde{\lambda}_{*},
$$

\footnotetext{
${ }^{11}$ An equivalent expression is given in appendix $\mathrm{A}$ of [52].

${ }^{12}$ This counting is the minimal form agreeing with explicit results up to six loops and satisfying the consistency conditions $N(N \lambda)^{p-1} N^{\left\lfloor\frac{1}{2}(p-3)\right\rfloor}(N \lambda)^{p^{\prime}} N^{\left\lfloor\frac{1}{2}\left(p^{\prime}-3\right)\right\rfloor} \leq(N \lambda)^{p+p^{\prime}-1} N^{\left\lfloor\frac{1}{2}\left(p+p^{\prime}-4\right)\right\rfloor}$ corresponding to inserting a $\lambda^{p^{\prime}}$ vertex graph for one of the vertices in the $\lambda^{p}$ graph.
} 
which is positive for $N \varepsilon<\frac{36}{\pi^{2}}$ and vanishes when $N \varepsilon=\frac{36}{\pi^{2}}$. In this large $N$ limit the $\beta$-function results in (6.9) also imply a UV fixed point

$$
\tilde{\lambda}_{* \mathrm{UV}} \approx \frac{4}{\pi^{2}}\left(3+\sqrt{9-\pi^{2} N \varepsilon / 4}\right) \rightarrow \frac{24}{\pi^{2}} \quad \text { as } \quad \varepsilon \rightarrow 0,
$$

which for $\varepsilon=0$ was considered in [53]. ${ }^{13}$ The result for $\tilde{\lambda}_{* \mathrm{UV}}$ extends to $\varepsilon<0$ although then at the IR fixed point $\tilde{\lambda}_{*}<0$ which is presumably unstable.

The $N$ dependence here is in contrast to the $O(N)$ model in $4-\varepsilon$ dimensions where the perturbative expansion gives contributions to $\beta_{\lambda}$ and $\gamma_{\phi}, \gamma_{\sigma}$ beyond one loop of the form $\lambda^{2}(N \lambda)^{p}$ and $\lambda(N \lambda)^{p}, p=1,2, \ldots$ For large $N$ in this case defining $\tilde{\lambda}=N \lambda$ then, to first order in $1 / N, \beta \tilde{\lambda}=-\varepsilon \tilde{\lambda}+\tilde{\lambda}^{2}+\frac{1}{N} \tilde{\lambda} f(\tilde{\lambda}), \gamma_{\phi} \sim \frac{1}{N} f_{\phi}(\tilde{\lambda}), \gamma_{\sigma} \sim \tilde{\lambda}+\frac{1}{N} f_{\sigma}(\tilde{\lambda})$ and at the fixed point $\tilde{\lambda}_{*} \sim \varepsilon-\frac{1}{N} f(\varepsilon)$ so that $\gamma_{\phi *} \sim \frac{1}{N} f_{\phi}(\varepsilon), \gamma_{\sigma *} \sim \varepsilon+\frac{1}{N}\left(-f(\varepsilon)+f_{\sigma}(\varepsilon)\right)$ where $f_{\phi}(\varepsilon)=\mathrm{O}\left(\varepsilon^{2}\right)$. The fixed point here is also unstable when $\varepsilon<0$.

Extending the discussion to lower dimension operators we may consider

$$
\begin{aligned}
V(\phi) \rightarrow & V(\phi)+\frac{1}{2} \sigma \phi^{2}+\frac{1}{2} \rho_{i j} \phi_{i} \phi_{j}+\frac{1}{2} \chi_{i} \phi_{i} \phi^{2}+\frac{1}{6} \tau_{i j k} \phi_{i} \phi_{j} \phi_{k} \\
& +\frac{1}{8} \xi\left(\phi^{2}\right)^{2}+\frac{1}{4} \mu_{i j} \phi_{i} \phi_{j} \phi^{2}+\frac{1}{24} \nu_{i j k l} \phi_{i} \phi_{j} \phi_{k} \phi_{l},
\end{aligned}
$$

with $\rho_{i j}, \tau_{i j k}, \mu_{i j}, \nu_{i j k l}$ symmetric and traceless. The results in (6.1) and (6.2) determine the anomalous dimensions. For the $O(N)$ symmetric theory determined by (6.4)

$$
\begin{array}{rlrl}
\gamma_{\sigma}^{(4)} & =\frac{16}{3}(N+2)(N+4) \lambda^{2}, & \gamma_{\rho}^{(4)} & =\frac{4}{3}(N+4)(N+8) \lambda^{2}, \\
\gamma_{\chi}^{(2)} & =2(N+4) \lambda, & \gamma_{\tau}^{(2)} & =4 \lambda, \\
\gamma_{\chi}^{(4)} & =-\frac{1}{2}(N+4)(25 N+242) \lambda^{2}, & \gamma_{\tau}^{(4)} & =\frac{1}{2}\left(7 N^{2}-6 N-424\right) \lambda^{2}, \\
\gamma_{\xi}^{(2)} & =8(N+4) \lambda, \quad \gamma_{\mu}^{(2)}=4(N+8) \lambda, & \gamma_{\nu}^{(2)}=16 \lambda, \\
\gamma_{\xi}^{(4)} & =-(N+4)\left(\frac{4}{3}(85 N+566)+\frac{1}{2}\left(N^{2}+18 N+116\right) \pi^{2}\right) \lambda^{2}, \\
\gamma_{\mu}{ }^{(4)} & =-\left(\frac{8}{3}\left(14 N^{2}+267 N+1132\right)+\left(N^{2}+44 N+232\right) \pi^{2}\right) \lambda^{2}, \\
\gamma_{\nu}{ }^{(4)} & =\left(\frac{4}{3}\left(5 N^{2}-78 N-968\right)-6(N+16) \pi^{2}\right) \lambda^{2} .
\end{array}
$$

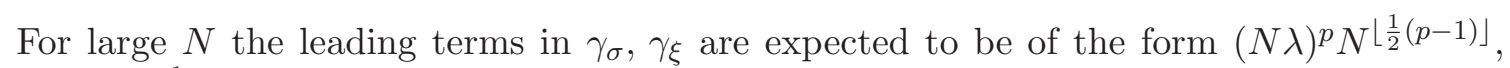
$(N \lambda)^{p} N^{\left\lfloor\frac{1}{2} p\right\rfloor}$. In the large $N$ limit for fixed $\tilde{\lambda}$ these results, together with [55], imply

$$
\begin{aligned}
& \gamma_{\phi}=\frac{1}{N^{2}} \frac{1}{6} \tilde{\lambda}^{2}+\mathrm{O}\left(\frac{1}{N^{3}}\right), \quad \gamma_{\sigma}=\frac{1}{N^{2}}\left(\frac{16}{3} \tilde{\lambda}^{2}-\frac{1}{2} \pi^{2} \tilde{\lambda}^{3}\right)+\mathrm{O}\left(\frac{1}{N^{3}}\right), \\
& \gamma_{\xi}=\frac{1}{N}\left(8 \tilde{\lambda}-\frac{1}{2} \pi^{2} \tilde{\lambda}^{2}\right)+\mathrm{O}\left(\frac{1}{N^{2}}\right) .
\end{aligned}
$$

\footnotetext{
${ }^{13} \mathrm{~A}$ UV fixed point for large $N$ was also proposed in [56, 57]. However [58, 59] showed for $N \rightarrow \infty$ and $d=3$, when the $\beta$-function vanishes, that a one loop effective potential was bounded below only when $\eta=8 \pi^{2} \tilde{\lambda} \leq 16 \pi^{2}$ or $\tilde{\lambda} \leq 2$. The UV fixed point in (6.12) for $\varepsilon=0$ is then in an unstable regime and is no longer relevant in this limit and there is a new scale invariant fixed point at $\tilde{\lambda}=2$. The situation for large but finite $N$ is less clear [60].
} 
At the fixed point the leading results for these anomalous dimensions are given by taking $\tilde{\lambda} \rightarrow \tilde{\lambda}_{*}$ as in (6.10). The large $N$ limit here is very different from that which interpolates between four and six dimensions. ${ }^{14}$ Only a finite number of terms contribute to any given order in $1 / N$ for fixed $N \varepsilon$. As is clear from (6.10) the $O(N)$ tricritical IR fixed point is not present for $N \varepsilon>\frac{36}{\pi^{2}}$. As argued in [63] there is a critical line $N=N_{c}(d)$ which involves another fixed point and [63] showed $N_{c}(d) \propto 1 / \varepsilon$ as $\varepsilon \rightarrow 0$. With tensorial scalar fields transforming under $O(N)^{5}$ there may be large $N$ limiting theories which may be analysed directly [64].

For two flavours the discussion in sections 2, 3 can be extended by taking a general potential of the form

$$
\begin{aligned}
V(\phi)= & \frac{1}{6 !}\left(\lambda_{1} \phi_{1}^{6}+\lambda_{2} \phi_{2}^{6}\right)+\frac{1}{2 \times 4 !}\left(g_{1} \phi_{1}^{4} \phi_{2}^{2}+g_{2} \phi_{1}^{2} \phi_{2}^{4}\right) \\
& +\frac{1}{5 !}\left(h_{1} \phi_{1}^{5} \phi_{2}+h_{2} \phi_{1} \phi_{2}^{5}\right)+\frac{1}{3 !^{2}} h \phi_{1}^{3} \phi_{2}^{3}
\end{aligned}
$$

with seven couplings $G_{I}=\left(\lambda_{1}, \lambda_{2}, g_{1}, g_{2}, h_{1}, h_{2}, h\right)$. Decomposing into representations of $O(2)$ give

$$
\begin{aligned}
& I_{1}=\lambda_{1}+\lambda_{2}+3 g_{1}+3 g_{2}, \\
& v_{6}=\left(\begin{array}{c}
\frac{1}{2}\left(\lambda_{1}-\lambda_{2}-15\left(g_{1}-g_{2}\right)\right) \\
3\left(h_{1}+h_{2}\right)-10 h
\end{array}\right), \\
& v_{2}=\left(\begin{array}{c}
\frac{1}{2}\left(\lambda_{1}-\lambda_{2}+g_{1}-g_{2}\right) \\
h_{1}+h_{2}+2 h
\end{array}\right), \quad v_{4}=\left(\begin{array}{c}
\frac{1}{4}\left(\lambda_{1}+\lambda_{2}-5\left(g_{1}+g_{2}\right)\right) \\
h_{1}-h_{2}
\end{array}\right),
\end{aligned}
$$

where $I_{1}$ is an invariant and $v_{2}, v_{4}, v_{6}$ are two-vectors transforming as in (3.9). The $O(2)$ invariant theory as in (6.4) corresponds to taking $v_{2}=v_{4}=v_{6}=0$ implying $h_{1}=h_{2}=h=$ $0, \lambda_{1}=\lambda_{2}=5 g_{1}=5 g_{2}=15 \lambda$. In a similar fashion to (3.3) there are five $O(2)$ invariants, in addition to $I_{1}$, which can be constructed from $v_{2}, v_{4}, v_{6}$ using the transformation (2.11).

Imposing restrictions on the $O(2)$ representation space formed by the couplings may reduce the RG flow to a sub manifold. Thus setting $v_{2}=v_{4}=0$ gives, since tensor products of $v_{6}$ cannot generate vectors transforming as $v_{2}, v_{4}$,

$$
\begin{aligned}
\beta_{I}(G) & =G_{I} a\left(g_{1}+g_{2}, h^{2}-g_{1} g_{2}\right)+G_{I}^{0} b\left(g_{1}+g_{2}, h^{2}-g_{1} g_{2}\right), \\
G_{I} & =\left(2 g_{1}+3 g_{2}, 3 g_{1}+2 g_{2}, g_{1}, g_{2},-h,-h, h\right), \quad G_{I}^{0}=(5,5,1,1,0,0,0),
\end{aligned}
$$

where $g_{1}+g_{2}, h^{2}-g_{1} g_{2}$ are two $O(2)$ invariants formed from $I_{1}, v_{6}^{2}$. Due to the restriction on the representations of the couplings the anomalous dimension matrix is diagonal and we must have

$$
\gamma_{i j}=\gamma\left(g_{1}+g_{2}, h^{2}-g_{1} g_{2}\right) \delta_{i j} .
$$

\footnotetext{
${ }^{14}$ However with the large $N$ dependence assumed above there might be an alternative large $N$ limit where $\lambda^{\prime}=N^{\frac{3}{2}} \lambda[61]$ and $\beta_{\lambda^{\prime}}=-2 \varepsilon \lambda^{\prime}+\lambda^{\prime} f\left(\lambda^{\prime 2}\right)+\mathrm{O}\left(N^{-\frac{1}{2}}\right)$ where $f\left(\lambda^{\prime 2}\right)=-\frac{1}{2} \pi^{2} \lambda^{\prime 2}+\ldots$ For $\varepsilon<0$ there is a UV fixed point in which $\lambda^{\prime} * \sqrt{-\frac{4}{\pi^{2}} \varepsilon}+\mathrm{O}(\varepsilon)$. The positive square root is chosen to ensure a stable potential and this fixed point is identical with (6.12) continued to $\varepsilon<0$ and taking $-N \varepsilon \gg 1$. In [62] it was suggested that $f\left(\lambda^{\prime 2}\right)$ should have a zero leading to an IR fixed point in the vicinity of three dimensions. Such a fixed point is not accessible with perturbative arguments but is motivated by the requirement that the UV fixed point should be annihilated by merging with an IR point for some $0<-\varepsilon<1$ so as to ensure a trivial theory in four dimensions.
} 
Perturbatively

$$
\begin{aligned}
& a(x, y)=-2 \varepsilon+\frac{40}{3} x-\left(\frac{5536}{15}+45 \pi^{2}\right) x^{2}+\frac{16}{15} y, \\
& b(x, y)=\frac{4}{3}\left(3 x^{2}+4 y\right)-x\left(15\left(12+\pi^{2}\right) x^{2}+4\left(74+9 \pi^{2}\right) y\right), \\
& \gamma(x, y)=\frac{1}{45}\left(7 x^{2}+8 y\right) .
\end{aligned}
$$

For $a(x, y)=b(x, y)=0$ we must take $y=-\frac{3}{4} x^{2}+\mathrm{O}\left(x^{3}\right)$ and at the fixed point then to $\mathrm{O}\left(\varepsilon^{2}\right)$

$$
x_{*}=\frac{3}{20} \varepsilon+\frac{9 \times 1387}{20000} \varepsilon^{2}+\frac{3^{5}}{3200} \pi^{2} \varepsilon^{2}, \quad y_{*}=-\frac{27}{1600} \varepsilon^{2} .
$$

However for real couplings $h, g_{1}, g_{2}$ we must have $-y \leq \frac{1}{4} x^{2}$ so this solution is not possible for this case

A similar reduction holds if $v_{2}=v_{6}=0$. In this case

$$
\begin{aligned}
\beta_{I}(G) & =G_{I} a\left(\lambda_{1}+3 g_{1}, y\right)+G_{I}^{0} b\left(\lambda_{1}+3 g_{1}, y\right), & y & =h_{1}^{2}-\left(\lambda_{1}-g_{1}\right) g_{1}, \\
G_{I} & =\left(\lambda_{1}, \lambda_{1}, g_{1}, g_{1}, h_{1},-h_{1}, 0\right), & G_{I}^{0} & =(5,5,1,1,0,0,0),
\end{aligned}
$$

with $\lambda_{1}+3 g_{1}, y$ invariants formed from $I_{1}, v_{4}^{2}$. Perturbative results give

$$
\begin{aligned}
& a(x, y)=-2 \varepsilon+\frac{20}{3} x-\frac{1}{30}\left(2248+225 \pi^{2}\right) x^{2}-\frac{1}{5}\left(848+75 \pi^{2}\right) y, \\
& b(x, y)=4 y-2\left(28+3 \pi^{2}\right) x y .
\end{aligned}
$$

The fixed point is realised to this order at

$$
y_{*}=0, \quad x_{*}=\frac{3}{10} \varepsilon+\frac{9}{20000}\left(2248+225 \pi^{2}\right) \varepsilon^{2} .
$$

The constraint $y=0$ can be realised by taking $h_{1}=g_{1}=0$ which corresponds to two decoupled theories or by taking $h_{1}=0, \lambda_{1}=g_{1}$, so that $\lambda_{1}+3 g_{1}=4 g_{1}$, which gives an equivalent theory. The anomalous dimension is again necessarily diagonal

$$
\gamma_{i j}=\gamma\left(\lambda_{1}+3 g_{1}, h_{1}^{2}-\left(\lambda_{1}-g_{1}\right) g_{1}\right) \delta_{i j}
$$

and from (6.3) to lowest order

$$
\gamma(x, y)=\frac{1}{90}\left(x^{2}+6 y\right)
$$

For both (6.18) and (6.22) there is a fixed point when $G_{I} \propto G_{I}^{0}$ giving the $O(2)$ invariant theory. In the first case it is necessary to take $g_{1}=g_{2}, h=0$, so that in (6.20) $x=2 g_{1}, y=-g_{1}^{2}$, and in the second case $h_{1}=0, \lambda_{1}=5 g_{1}$, and in (6.23) $x=8 g_{1}, y=$ $-4 g_{1}^{2}$. The results can be reduced to $\beta_{\lambda}$ for $\lambda_{1}=5 g_{1}=15 \lambda$ and agrees with (6.5) and (6.7) for $N=2$ and (6.20), (6.26) both give $\gamma=4 \lambda^{2}$ in agreement with (6.6) when $N=2$.

In a similar fashion to four dimensions we may consider perturbations by operators $d_{i j k l m n} \phi^{i} \phi^{j} \phi^{k} \phi^{l} \phi^{m} \phi^{n}$ and $\phi^{2} d_{i j k l} \phi^{i} \phi^{j} \phi^{k} \phi^{l}$ with $d_{i j k l m n}, d_{i j k l}$ symmetric and traceless. For 
the former to lowest order at the $O(N)$ symmetric fixed point $\kappa=\Delta-d=-\frac{2(3 N+2)}{3 N+22} \varepsilon$, so this is always relevant. For the latter $\kappa=-\frac{2(N-14)}{3 N+22} \varepsilon$ so this is also relevant when $N>14$. As an example we consider a breaking $O(N) \rightarrow \mathcal{S}_{N} \ltimes \mathbb{Z}_{2}^{N}$, where, for a closed RG flow, it is necessary to introduce two additional couplings

$$
V(\phi)=\frac{1}{48} \lambda\left(\phi^{2}\right)^{3}+\frac{1}{48} g \phi^{2} \sum_{i} \phi_{i}^{4}+\frac{1}{6 !} h \sum_{i} \phi_{i}^{6} .
$$

The $\beta$-functions may be read off from (6.1),

$$
\begin{aligned}
& \beta_{\lambda}=-2 \varepsilon \lambda+4\left((3 N+22) \lambda^{2}+12 \lambda g+g^{2}\right), \\
& \beta_{g}=-2 \varepsilon g+4\left(2(N+18) \lambda g+2 \lambda h+13 g^{2}+\frac{2}{3} g h\right), \\
& \beta_{h}=-2 \varepsilon h+20\left(4 \lambda h+(N+32) g^{2}+8 g h+\frac{1}{3} h^{2}\right) .
\end{aligned}
$$

As a consequence of the $\mathcal{S}_{N} \ltimes \mathbb{Z}_{2}^{N}$ symmetry the anomalous dimension matrix is proportional to $\delta_{i j}$ and (6.6) is extended to

$$
\gamma^{(4)}=\frac{1}{6}(N+2)(N+4) \lambda^{2}+(N+4) \lambda g+\frac{1}{6}(N+14) g^{2}+\frac{1}{3}(\lambda+g) h+\frac{1}{90} h^{2} .
$$

For this theory there is of course a fixed point with $O(N)$ symmetry for $g_{*}=h_{*}=0$ and where $\lambda_{*}$ is given by (6.8). For this the stability matrix from (6.28) becomes

$$
M=-2 \varepsilon \mathbb{1}+8 \lambda_{*}\left(\begin{array}{ccc}
3 N+22 & 0 & 0 \\
6 & N+18 & 0 \\
0 & 1 & 10
\end{array}\right) .
$$

The eigenvalues are $2 \varepsilon,-\frac{N-14}{3 N+22} 2 \varepsilon,-\frac{3 N+2}{3 N+22} 2 \varepsilon$ with left eigenvectors $\left(\begin{array}{lll}1 & 0 & 0\end{array}\right),\left(\begin{array}{lll}-\frac{3}{N+2} & 1 & 0\end{array}\right)$, $\left(\frac{2}{(N+8)(N+2)}-\frac{1}{N+8} 1\right)$ respectively. In consequence there is always a relevant operator and the $O(N)$ fixed point is unstable. When $N=2$ the three terms in (6.27) are not independent, in comparison with (6.16) $\lambda_{1}=\lambda_{2}=15(\lambda+g)+h, g_{1}=g_{2}=3 \lambda+g$ with the other couplings, which break the reflection symmetry under $\phi_{i} \rightarrow-\phi_{i}$, zero. For this case (6.29) gives $\gamma^{(4)}=\frac{1}{90} \lambda_{1}^{2}+\frac{1}{6} g_{1}^{2}$.

There is a trivial decoupled fixed point for $\lambda_{*}=g_{*}=0, h_{*}=\frac{3}{10} \varepsilon$. In this case

$$
M=-2 \varepsilon \mathbb{1}+8 h_{*}\left(\begin{array}{ccc}
0 & 1 & 10 \\
0 & \frac{1}{3} & 20 \\
0 & 0 & \frac{5}{3}
\end{array}\right),
$$

with eigenvalues $2 \varepsilon,-\frac{6}{5} \varepsilon,-2 \varepsilon$ with eigenvectors $(0,0,1),(0,1,-15),(1,-3,30)$.

Other fixed points for non zero $\lambda, g, h$ can be found if we define $x=g / \lambda, y=h / \lambda$ and then from (6.28) we must require

$$
\begin{aligned}
& F(x, y) \equiv\left(x^{2}-x+N-14\right) x-\frac{2}{3}(x+3) y=0 \\
& G(x, y) \equiv\left(x^{2}-28 x+3 N+2\right) y-5(N+32) x^{2}-\frac{5}{3} y^{2}=0 .
\end{aligned}
$$


For $x=-3$ then the first equation is satisfied for $N=2$ and then the second equation determines $y=30, \frac{153}{3}$. These give $\lambda_{*}=\frac{1}{2} \varepsilon, g_{*}=-\frac{3}{2} \varepsilon, h_{*}=15 \varepsilon, \frac{153}{5} \varepsilon$ implying in (6.16) with $h=h_{1}=h_{2}=0$ fixed points for $\lambda_{1}, g_{1}=0,0$ and $0, \frac{3}{10} \varepsilon$ respectively. For $x \neq-3$ then $F(x, y)=0$ may be solved for $y$ and $G(x, y)$ determines a quintic polynomial whose zeros determine the non zero $x$ giving rise to fixed points. For $N=2$ this gives $x=-\frac{8}{3}$ and hence $y=\frac{80}{3}$ and then $\lambda_{*}=\frac{9}{56} \varepsilon, g_{*}=-\frac{3}{7} \varepsilon, h_{*}=\frac{30}{7} \varepsilon$. This corresponds to taking $\lambda_{1}=5 g_{1}=\frac{15}{56} \varepsilon$ which is the $O(2)$ fixed point. For large $N$ the quintic has three real roots with $x= \pm \frac{1}{3} N^{\frac{1}{2}}$ and $x=-18$, correspondingly $y=\frac{5}{3} N$ and $y=\frac{9}{5} N$, giving fixed points

$$
\begin{aligned}
& \lambda_{*}=\frac{9}{56 N} \varepsilon, \quad g_{*}= \pm \frac{3}{56 \sqrt{N}} \varepsilon, \quad h_{*}=\frac{15}{56} \varepsilon, \\
& \lambda_{*}^{\prime}=\frac{1}{6 N} \varepsilon, \quad \quad g_{*}^{\prime}=-\frac{3}{N} \varepsilon, \quad h_{*}^{\prime}=\frac{3}{10} \varepsilon .
\end{aligned}
$$

The stability matrices at these fixed points, to leading order in $N$, are given by

$$
\frac{\varepsilon}{7}\left(\begin{array}{ccc}
13 & \pm 3 \sqrt{N} & 0 \\
\pm 3 / \sqrt{N} & 0 & \pm 15 \sqrt{N} \\
0 & \pm 1 / \sqrt{N} & 11
\end{array}\right), \quad 2 \varepsilon\left(\begin{array}{ccc}
1 & -\frac{54}{5} & 12 \\
0 & \frac{1}{15} & -36 \\
0 & 0 & 1
\end{array}\right)
$$

with eigenvalues $2 \varepsilon, \frac{1}{7}(5 \pm \sqrt{46}) \varepsilon$ and $2 \varepsilon, 2 \varepsilon, \frac{2}{15} \varepsilon$, which is therefore stable, respectively. However for $2<N<14035$ there is just one real root, corresponding to $x \approx \frac{1}{3} N^{\frac{1}{2}}, y \approx \frac{5}{3} N$ as $N$ becomes large, and in this case there is no stable fixed point.

For the RG flow there is a corresponding $a$-function (results for theories with fermions are contained in $[65,66])$ given by

$$
\begin{aligned}
A=N( & 5(N+2)(N+4)\left(\lambda \beta_{\lambda}-\varepsilon \lambda^{2}\right)+5(N+14)\left(g \beta_{g}-\varepsilon g^{2}\right)+\frac{1}{3}\left(h \beta_{h}-\varepsilon h^{2}\right) \\
& +15(N+4)\left(\lambda \beta_{g}+g \beta_{\lambda}-2 \varepsilon \lambda g\right) \\
& \left.+5\left(\lambda \beta_{h}+h \beta_{\lambda}+g \beta_{h}+h \beta_{g}-2 \varepsilon \lambda h-2 \varepsilon g h\right)\right)
\end{aligned}
$$

with $\beta_{\lambda}, \beta_{g}, \beta_{h}$ given by (6.28). This satisfies

$$
\left(\begin{array}{l}
\partial_{\lambda} \\
\partial_{g} \\
\partial_{h}
\end{array}\right) A=N\left(\begin{array}{ccc}
15(N+2)(N+4) & 45(N+4) & 15 \\
45(N+4) & 15(N+14) & 15 \\
15 & 15 & 1
\end{array}\right)\left(\begin{array}{c}
\beta_{\lambda} \\
\beta_{g} \\
\beta_{h}
\end{array}\right)
$$

and so $A$ decreases under RG flow towards IR fixed points as the effective metric on the couplings $\lambda, g, h$ is positive definite for $N>2$, for $N=2$ the metric has a zero eigenvalue reflecting the redundancy of the couplings $\lambda, g, h$. From (6.36) and (6.29) the lowest order results satisfy

$$
\left.A\right|_{\beta_{\lambda}=\beta_{g}=\beta_{h}=0}=-\frac{5}{6} N \gamma^{(4)} \varepsilon .
$$

For the $O(N)$ and decoupled fixed points corresponding to (6.30) and (6.31)

$$
A_{O(N)}=-\frac{5 N(N+2)(N+4)}{4(3 N+22)^{2}} \varepsilon^{3}, \quad A_{\text {decoupled }}=-\frac{3 N}{100} \varepsilon^{3} .
$$


In the large $N$ limit corresponding to (6.33)

$$
A_{*}=-\frac{75 N}{448} \varepsilon^{3}, \quad A_{*}^{\prime}=-\frac{38 N}{225} \varepsilon^{3}<A_{*},-\frac{5 N}{36} \varepsilon^{3} .
$$

In section 5 we considered theories in $4-\varepsilon$ dimensions based on a potential of the form (3.1).

\section{$7 \quad$ Multiple couplings in $4-\varepsilon$ dimensions}

The discussion of possible fixed points becomes tractable by limiting the number of fields or the number of couplings. In section 5 we considered theories in $4-\varepsilon$ dimensions based on a potential of the form (3.1) which had two couplings. There are many examples in the literature in which theories with three couplings are considered in the $\varepsilon$-expansion [44, 6770]. For such examples $V$ can be expressed as a sum of terms involving symmetric traceless tensors $d_{u, i j k l}$ as well as an $O(N)$ symmetric term then the treatment in section 5 may be straightforwardly extended with couplings $\left\{\lambda, g^{u}\right\}, u=1, \ldots, p$. For the theory to be closed under RG flow we modify (5.2) to

$$
d_{u, i j m n} d_{v, k l m n}=\frac{1}{N-1} a_{u v}\left(\frac{1}{2} N\left(\delta_{i k} \delta_{j l}+\delta_{i l} \delta_{j k}\right)-\delta_{i j} \delta_{k l}\right)+b_{u v}{ }^{w} d_{w, i j k l},
$$

where $\left[a_{u v}\right]$ is positive definite and

$$
b_{u v w}=b_{u v}^{x} a_{x w}=b_{(u v w)} .
$$

It is convenient to use $a_{u v}$, and its matrix inverse, to lower and raise indices for the couplings $\left\{g^{u}\right\}$. Of course (7.1) may be extended as in (5.59), but this is not pursued here.

The lowest order $\beta$-functions are just a simple modification of (5.9)

$$
\begin{aligned}
\beta_{\lambda} & =-\varepsilon \lambda+(N+8) \lambda^{2}+g^{2}, \quad g^{2}=a_{u v} g^{u} g^{v}, \\
\beta_{g}{ }^{u} & =-\varepsilon g^{u}+12 \lambda g^{u}+3 g^{v} g^{w} b_{v w}{ }^{u},
\end{aligned}
$$

and associated $a$-function from (5.10) becomes

$$
A=N(N+2)\left(-\frac{3}{2} \varepsilon \lambda^{2}-\frac{1}{4} \varepsilon g^{2}+(N+8) \lambda^{3}+3 \lambda g^{2}+\frac{1}{2} b_{u v w} g^{u} g^{v} g^{w}\right) .
$$

At two loops we can easily extend (5.23) to the multi-coupling case and also at three loops (5.28) with an appropriate generalisation of (5.27). The couplings $\left(g^{1}, \ldots, g^{p}\right) \in \mathbb{R}^{p}$ define a vector space spanned by the polynomials $d_{u, i j k l} \phi^{i} \phi^{j} \phi^{k} \phi^{l}$. If there is a subspace $V \subset \mathbb{R}^{p}$ such that for any $g^{u}, g^{\prime v} \in V$ then $g^{u} g^{\prime v} b_{u v}{ }^{w} \in V$ the RG flow may be restricted to couplings belonging to $V$.

It is easy to see that there are no fixed points with $\lambda_{*}=0$ other than the trivial Gaussian one. Hence it is sufficient to consider $x^{u}=g^{u} / \lambda$. Under RG flow from (7.3) to lowest order

$$
\frac{\mathrm{d}}{\mathrm{d} t} \lambda=\lambda\left(\varepsilon-\lambda f_{\lambda}(x)\right), \quad \frac{\mathrm{d}}{\mathrm{d} t} x^{u}=\lambda f^{u}(x),
$$


where

$$
f_{\lambda}(x)=N+8+x^{2}, \quad f^{u}(x)=\left(N-4+x^{2}\right) x^{u}-3 b_{v w}{ }^{u} x^{v} x^{w} .
$$

Higher order contributions in (7.5) involve contributions at $\ell$ loops of the form $\lambda^{\ell+1} f_{\ell, \lambda}(x)$ and $\lambda^{\ell} f_{\ell}^{u}(x)$. Perturbative fixed points are then obtained iteratively by first solving $f^{u}\left(x_{*}\right)=0$ giving $\lambda_{*}=\varepsilon / f_{\lambda}\left(x^{*}\right)+\mathrm{O}\left(\varepsilon^{2}\right)$.

At any fixed point, where the lowest order $\beta$-functions in (7.3) vanish, there is a similar bound to (5.20)

$$
\begin{aligned}
& A_{*}=-\frac{1}{2} N(N+2) \frac{1+\frac{1}{6} x_{*}^{2}}{\left(N+8+x_{*}^{2}\right)^{2}} \varepsilon^{3} \geq-\frac{1}{48} N \varepsilon^{3}=A_{* \text { bound }}, \\
& x_{*}^{u}=g_{*}^{u} / \lambda_{*}, \quad x_{*}{ }^{2}=a_{u v} x_{*}{ }^{u} x_{*}^{v} .
\end{aligned}
$$

Any $\left\{\lambda, g^{u}\right\}$ such that $A<-\frac{1}{48} N \varepsilon^{3}$ cannot then flow to a fixed point accessible in the $\varepsilon$-expansion. The bound in (7.7) arises when

$$
x_{*}^{2}=N-4,
$$

and so requires $N>4$. To analyse this further we consider the stability matrix at the fixed point

$$
M=\left(\begin{array}{cc}
-\varepsilon+2(N+8) \lambda_{*} & 12 g_{*}{ }^{v} \\
2 g_{* u} & \left(-\varepsilon+12 \lambda_{*}\right) \delta_{u}{ }^{v}+6 b_{u w}{ }^{v} g_{*}{ }^{w}
\end{array}\right) .
$$

The eigenvalue problem may be simplified by noting that

$$
M\left(\begin{array}{c}
\lambda_{*} \\
\frac{1}{6} g_{* v}
\end{array}\right)=\varepsilon\left(\begin{array}{c}
\lambda_{*} \\
\frac{1}{6} g_{* u}
\end{array}\right), \quad\left(\lambda_{*} g_{*}^{u}\right) M=\varepsilon\left(\lambda_{*} g_{*}^{v}\right),
$$

so that $\varepsilon$ is always an eigenvalue for this class of theories, and also

$$
M\left(\begin{array}{c}
-g_{*}^{w} v_{w} \\
\lambda_{*} v_{v}
\end{array}\right)=\kappa\left(\begin{array}{c}
-g_{*}^{w} v_{w} \\
\lambda_{*} v_{u}
\end{array}\right), \quad\left(-\frac{1}{6} u^{u} g_{* u} \lambda_{*} u^{u}\right) M=\kappa\left(-\frac{1}{6} u^{u} g_{* u} \lambda_{*} u^{v}\right),
$$

where $\kappa$ and $v_{r}, u^{r}$ are solutions of the reduced eigenvalue problems

$$
-\lambda_{*} \partial_{u} f^{s}\left(x_{*}\right) v_{v}=\kappa v_{u}, \quad-\lambda_{*} u^{u} \partial_{u} f^{v}\left(x_{*}\right)=\kappa u^{v},
$$

for $f^{u}(x)$ given in (7.6) and where $x_{*}$ is determined by $f^{u}\left(x_{*}\right)=0$, corresponding to a fixed point where (7.3) vanish. The results (7.10) and (7.11) with (7.12) generalise (5.19) in the two coupling case. In general there are $p$ eigenvalues $\kappa$ and associated eigenvectors. To verify (7.11) it is necessary to show that (7.12) implies

$$
\lambda_{*}\left(4-N+x_{*}^{2}\right) x_{*}^{u} v_{u}=\kappa x_{*}^{u} v_{v} .
$$

In consequence when (7.8) is satisfied there is a zero eigenvalue of the stability matrix as expected when two fixed points annihilate.

Assuming $\lambda_{*}>0$ it is easy to see that stable fixed points correspond to finding local maxima of

$$
F(x)=\frac{1}{2}(N-4) x^{2}+\frac{1}{4}\left(x^{2}\right)^{2}-b_{r s t} x^{r} x^{s} x^{t} .
$$


For $N<4$ manifestly there is a maximum at $x=0$. In general when $N>4$ and the origin is a minimum and $F\left(x_{*}\right)=\frac{1}{6}(N-4) x_{*}{ }^{2}-\frac{1}{12}\left(x_{*}{ }^{2}\right)^{2} \leq \frac{1}{12}(N-4)^{2}$. The function $F(x)$ on $\mathbb{R}^{p}$ can reduced to one on $S^{p} \simeq \mathbb{R}^{p} \cup\{\infty\}$ by modifying $F(x)$ for very large $x^{2}$ so that as $x \rightarrow \infty F(x) \rightarrow C$, a finite constant, and then $F(\infty)$ becomes the maximum of $F$. On $S^{p}$ $F(x)$ is a smooth bounded function with at least one maximum and one minimum. We may use baby Morse theory to constrain the stationary points of $F$ (in embryonic form analogous results were found in $[71,72])$. Assuming $F$ has $C_{t}$ stationary points at finite $x$ with non degenerate Hessians and $t=0,1, \ldots, p$ negative eigenvalues $\left(C_{0}, C_{p}\right.$ are the number of minima, maxima) then

$$
C_{t}-C_{t-1}+\cdots+(-1)^{t} C_{0} \geq(-1)^{t}, t<p, \quad \sum_{t=0}^{p}(-1)^{t} C_{t}+(-1)^{p}=1+(-1)^{p}
$$

where we add in the second identity the contribution of the maxima at infinity separately. A perfect Morse function on $S^{p}$, saturating the inequalities in (7.15), has just one minimum and one maximum and no other stationary points. This is realised if there is just a single minimum for finite $x$ in (7.14) giving $C_{0}=1, C_{p}=0$. If $b_{\max }=\left.\max \left(b_{r s t} e^{r} e^{s} e^{t}\right)\right|_{e^{2}=1}$ this holds if $N>4$ and $b_{\max }^{2}<\frac{4}{9}(N-4)$. For a stable fixed point it is necessary that $C_{p} \geq 1$. If a new fixed point is generated by varying the parameters this must correspond to $\Delta C_{q}=\Delta C_{q+1}=1$ for some $q$.

As an illustration we may consider a theory [73] with $p=2$ where $\phi_{i}=\left(\varphi_{r}, \psi_{r}\right)$, $r=1, \ldots, n, N=2 n, \varphi^{2}=\sum_{r} \varphi_{r}^{2}, \psi^{2}=\sum_{r} \psi_{r}^{2}$, by taking

$$
\begin{aligned}
& d_{1, i j k l} \phi_{i} \phi_{j} \phi_{k} \phi_{l}=\sum_{r}\left(\varphi_{r}^{4}+\psi_{r}^{4}\right)-\frac{3}{2(n+1)}\left(\varphi^{2}+\psi^{2}\right)^{2}, \\
& d_{2, i j k l} \phi_{i} \phi_{j} \phi_{k} \phi_{l}=\sum_{r} 2 \varphi_{r}^{2} \psi_{r}^{2}-\frac{1}{2(n+1)}\left(\varphi^{2}+\psi^{2}\right)^{2} .
\end{aligned}
$$

This extends the cubic fixed point theory each term in (7.16) has a symmetry $\mathcal{S}_{n} \ltimes D_{4}{ }^{n}$, with the dihedral group $D_{4}$ generated by $\left(\varphi_{r}, \psi_{r}\right) \rightarrow\left(-\varphi_{r}, \psi_{r}\right),\left(\psi_{r}, \varphi_{r}\right)$.

By considering $\pi / 4$ rotations of $\left(\varphi_{r}, \psi_{r}\right)$ theories related by transformations of the couplings of the form

$$
\left(\begin{array}{ll}
g^{1} & g^{2}
\end{array}\right) \rightarrow\left(\begin{array}{ll}
g^{1} & g^{2}
\end{array}\right)\left(\begin{array}{cc}
\frac{1}{2} & \frac{3}{2} \\
\frac{1}{2} & -\frac{1}{2}
\end{array}\right)
$$

are equivalent. It is straightforward to obtain $a_{r s}=a_{s r}, b_{r s}{ }^{t}=b_{s r}{ }^{t}$ in (7.1) for this case which are given by

$$
\begin{aligned}
& a_{11}=\frac{2 n-1}{2(n+1)^{2}}, \quad a_{12}=-\frac{1}{2(n+1)^{2}}, \quad a_{22}=\frac{2 n+1}{6(n+1)^{2}}, \\
& b_{11}^{1}=\frac{n-1}{n+1}, \quad b_{11}^{2}=0, \quad b_{12}{ }^{1}=-\frac{1}{3(n+1)}, \quad b_{12}^{2}=\frac{n-2}{3(n+1)}, \quad b_{22}^{1}=\frac{1}{9}, \quad b_{22}^{2}=\frac{2(2 n-1)}{9(n+1)} .
\end{aligned}
$$


These results satisfy (7.2). The functions $f^{u}\left(x^{1}, x^{2}\right)$ in (7.6) are given by

$$
\begin{aligned}
f^{1}(x, y)= & \frac{1}{(n+1)^{2}}\left(\frac{1}{2}(2 n-1) x^{2}+\frac{1}{6}(2 n+1) y^{2}-x y\right) x \\
& +\frac{1}{n+1}(2 y-3(n-1) x) x-\frac{1}{3} y^{2}+2(n-2) x, \\
f^{2}(x, y)= & \frac{1}{(n+1)^{2}}\left(\frac{1}{2}(2 n-1) x^{2}+\frac{1}{6}(2 n+1) y^{2}-x y\right) y \\
& -\frac{1}{n+1}\left(2(n-2) x+\frac{2}{3}(2 n-1) y\right) y+2(n-2) y,
\end{aligned}
$$

Finding fixed points by solving the two cubics $f^{1}\left(x^{1}, x^{2}\right)=f^{2}\left(x^{1}, x^{2}\right)=0$ gives seven solutions which, at lowest order in the $\varepsilon$-expansion, are given by

$$
\begin{aligned}
& \left(x_{*}^{1}, x_{*}^{2}\right)=(0,0), \\
& (2,0)(n+1), \\
& (1,3)(n+1), \\
& (2,0) \frac{(n-2)(n+1)}{2 n-1} \text {, } \\
& (1,3) \frac{(n-2)(n+1)}{2 n-1} \text {, } \\
& (1,1) \frac{3}{2}(n+1) \text {, } \\
& -\left[\lambda_{*} \partial_{r} f^{s}\right]=-\left(\begin{array}{cc}
1 & 0 \\
0 & 1
\end{array}\right) \frac{n-2}{n+4} \varepsilon, \\
& -\left(\begin{array}{ll}
1 & 0 \\
0 & 1
\end{array}\right) \frac{1}{3} \varepsilon, \\
& \left(\begin{array}{cc}
1 & 0 \\
-2 /(2 n-1) & -1
\end{array}\right) \frac{n-2}{3 n} \varepsilon, \\
& -\left(\begin{array}{cc}
1 & 3 \\
1 & -1
\end{array}\right) \frac{1}{10} \varepsilon, \\
& \left(\kappa_{1}, \kappa_{2}\right)=-(1,1) \frac{n-2}{n+4} \varepsilon, \\
& -(1,1) \frac{1}{3} \varepsilon \text {, } \\
& (1,-1) \frac{n-2}{3 n} \varepsilon, \\
& (1,-1) \frac{1}{5} \varepsilon \text {, } \\
& A_{*}=-\frac{n(n+1)}{2(n+4)^{2}} \varepsilon^{3} \text {, } \\
& -\frac{n}{27} \varepsilon^{3} \\
& -\frac{n}{27} \varepsilon^{3}, \\
& -\frac{(n+1)(2 n-1)}{54 n} \varepsilon^{3} \\
& -\frac{(n+1)(2 n-1)}{54 n} \varepsilon^{3} \text {, } \\
& -\frac{n}{25} \varepsilon^{3} \\
& -\left(\begin{array}{ll}
1 & 0 \\
0 & 1
\end{array}\right) \frac{1}{3} \varepsilon, \\
& \left(\begin{array}{c}
-n-1 \\
n
\end{array} \underset{n+1}{3(n-2)}\right) \frac{n-2}{3 n(2 n-1)} \varepsilon, \\
& \left(\begin{array}{ll}
1 & 0 \\
0 & 1
\end{array}\right) \frac{n-2}{5 n-4} \varepsilon, \\
& -(1,1) \frac{1}{3} \varepsilon, \\
& (1,-1) \frac{n-2}{3 n} \varepsilon, \\
& (1,1) \frac{n-2}{5 n-4} \varepsilon \text {, } \\
& -\frac{(n-1) n(2 n-1)}{2(5 n-4)^{2}} \varepsilon^{3} .
\end{aligned}
$$

There are seven fixed points such that in (7.15) $C_{0}=C_{1}=3, C_{2}=1$. The first fixed point is the usual $O(2 n)$ invariant Heisenberg fixed point, the second two are equivalent 
under (7.17) and correspond to $N$ decoupled Ising models, the next two are also equivalent and represent the hypercubic fixed point discussed in the two coupling case. The last two fixed points are not equivalent since each is invariant under (7.17). In this case the $d$-terms combine to $\sum_{r}\left(\varphi_{r}^{2}+\psi_{r}^{2}\right)^{2}-\frac{2}{n+1}\left(\varphi^{2}+\psi^{2}\right)^{2}$ and the symmetry is enhanced to $\mathcal{S}_{n} \ltimes O(2)^{n}$. These fixed points are identical to the $M N$ theory starting from (5.90) when $m=2$, the results for $A_{*}$ are identical to (5.98) with $m=2$. The final fixed point is stable and has the least value of $A_{*}$. Results for the stability matrix eigenvalues for each fixed point above are given to three loop order in [69], of course for equivalent theories the expressions are the same. All the fixed points in this three coupling theory correspond to ones obtained by RG flow starting from the trivial Gaussian fixed point in just single or double coupling theories.

An extension to models with arbitrarily many couplings which have stable fixed points can be achieved by adapting results due to Michel [74] to the formalism described here (a related discussion is contained in [75]). We take $\phi_{i}=\varphi_{a_{1}, \ldots, a_{p}, r}, a_{u}=1,2, \ldots, m_{u}$, where $r=1, \ldots, n$ and $u=1, \ldots, p$ and define

$$
\begin{aligned}
d_{u, i j k l} \phi_{i} \phi_{j} \phi_{k} \phi_{l} & =\sum_{a_{u+1}, \ldots, a_{p}, r}\left(\sum_{a_{1}, \ldots, a_{u}} \varphi_{a_{1}, \ldots, a_{p}, r}\right)^{2}-\frac{M_{u}+2}{N+2}\left(\varphi^{2}\right)^{2}, \\
M_{u} & =\prod_{1}^{u} m_{v}, \quad N=\prod_{1}^{p} m_{u} n, \quad m_{u} \geq 2 \text { if } u>1 .
\end{aligned}
$$

This is invariant under $\mathcal{S}_{N / M_{u}} \ltimes O\left(M_{u}\right)^{N / M_{u}} \in O(N)$. There are then $p$ couplings $g^{u}$ which, along with $\lambda$, define a closed manifold under $\mathrm{RG}$ flow. Each $\left(m_{1}, \ldots, m_{p}\right)$ represents a different theory with different symmetries although as will be shown they may flow to common fixed points. For $m_{1}=1, m_{2}=2 d_{1}, d_{2}$ are equivalent to (7.16), the couplings are related to the previous case by $g^{1} \rightarrow g^{1}-g^{2}, g^{2} \rightarrow g^{2}$. Scalar potentials formed by linear combinations of terms of the form (7.21) include various theories which have been considered in the literature. In (7.1) the non zero contributions are given by

$$
a_{u v}=a_{v u}=\frac{2\left(M_{u}+2\right)\left(N-M_{v}\right)}{3(N+2)}, \quad v \geq u
$$

and

$$
\begin{aligned}
& b_{u v}{ }^{u}=b_{v u}{ }^{u}=\frac{2\left(N-M_{v}\right)}{3(N+2)}, \quad b_{u v}^{v}=b_{v u}{ }^{v}=\frac{(N-4)\left(M_{u}+2\right)}{9(N+2)}, \quad v>u, \\
& b_{u u}{ }^{u}=\frac{1}{9(N+2)}\left((N-4)\left(M_{u}+2\right)+6\left(N-M_{u}\right)\right) .
\end{aligned}
$$

It is easy to verify (7.2).

From (7.6)

$$
\begin{aligned}
f^{u}(x) & =x^{u}\left(N-4+x^{2}-\frac{1}{3(N+2)}\left((N-4) Y^{u}+6 X^{u}\right)\right), \\
X^{u} & =\left(N-M_{u}\right) x^{u}+2 \sum_{v>u}\left(N-M_{v}\right) x^{v}, \quad Y^{u}=\left(M_{u}+2\right) x^{u}+2 \sum_{v<u}\left(M_{v}+2\right) x^{v}, \\
x^{2} & =\frac{2}{3(N+2)^{2}} \sum_{u}\left(M_{u}+2\right) x^{u} X^{u} .
\end{aligned}
$$


Hence the fixed points at lowest order in $\varepsilon$ are given by

$$
x^{u}=0 \text { or } N-4+x^{2}-\frac{1}{3(N+2)}\left((N-4) Y^{u}+6 X^{u}\right)=0 \text { for each } u .
$$

Setting all $x^{u}$ to zero gives the $O(N)$ symmetric Heisenberg fixed point. If $x^{u_{1}}, x^{u_{2}}, \ldots, x^{u_{q}}$, $u_{1}<u_{2}<\cdots<u_{q}$, are non zero, solutions of the fixed point equations can be found by taking

$$
x_{*}^{u_{i}}=\frac{1}{B} \frac{(N-4)(N+2)}{2\left(M_{u_{i}}-4\right)}(-1)^{i}, \quad i=1, \ldots, q,
$$

where $B$ satisfies

$$
\begin{aligned}
B^{2}+\left(\frac{1}{6}(N+2)-2 S_{q}\right) B & =\frac{1}{6}(N-4) \sum_{i=1}^{q} \frac{M_{u_{i}}+2}{M_{u_{i}}-4}\left(\frac{N-M_{u_{i}}}{M_{u_{i}}-4}+2 \sum_{j>i}(-1)^{i+j} \frac{N-M_{u_{j}}}{M_{u_{j}}-4}\right) \\
& =S_{q}^{2}-\frac{1}{6}(N+2) S_{q}, \quad S_{q}=\sum_{i=1}^{q}(-1)^{i} \frac{N-M_{u_{i}}}{M_{u_{i}}-4} .
\end{aligned}
$$

In consequence there are two solutions for $B$

$$
B=S_{q}, \quad S_{q}-\frac{1}{6}(N+2),
$$

giving at the fixed point

$$
\lambda_{* 1}=\frac{6 S_{q}}{12 S_{q}-N+4} \frac{1}{N+2} \varepsilon, \quad \lambda_{* 2}=\frac{6 S_{q}-N-2}{12 S_{q}-N-8} \frac{1}{N+2} \varepsilon .
$$

For stability it is necessary that $\lambda_{* 1}$ or $\lambda_{* 2}$ are positive, thus for the first fixed point, assuming $N>4$,

$$
S_{q}<0 \quad \text { or } \quad 12 S_{q}>N-4
$$

For the two cases in (7.28)

$$
A_{* 1}=\left(\left(\frac{N-4}{12 S_{q}-N+4}\right)^{2}-1\right) \frac{N}{48} \varepsilon^{3}, \quad A_{* 2}=\left(\left(\frac{N-4}{12 S_{q}-N-8}\right)^{2}-1\right) \frac{N}{48} \varepsilon^{3} .
$$

In general $M_{u_{1}}<M_{u_{2}}<\cdots<M_{u_{q}}<N$ so that if $M_{u_{1}}>4$ then $S_{q}<0$. For $S_{q}>0$ it is necessary that $M_{u_{1}}<4$ requiring $m_{1}<4$. For $q$ even

$$
S_{q}=(N-4) \sum_{i=1}^{q}(-1)^{i} \frac{1}{M_{u_{i}}-4} .
$$

Besides the Heisenberg fixed point there are for each $q=1, \ldots, p\left(\begin{array}{l}p \\ q\end{array}\right)$ choices and hence $2\left(2^{p}-1\right)$ fixed points with some non zero $x_{*}{ }_{*}$. These results for $p=1$ are identical to (5.96) and (5.98) for $m_{1} \rightarrow m$.

For each fixed point given by (7.26) and (7.28), $q=1, \ldots, p$ we may determine the $p$ eigenvalues of the stability matrix by solving (7.12) with (7.24). The results take the simple form for the two cases in (7.28)

$$
\kappa_{1}=\frac{N-4}{12 S_{q}-N+4}\left(1_{p-t},-1_{t}\right) \varepsilon, \quad \kappa_{2}=\frac{N-4}{12 S_{q}-N-8}\left(1_{p-t-1},-1_{t+1}\right) \varepsilon .
$$


The number of negative modes is given by

$$
t= \begin{cases}p-u_{1}+u_{2}-\cdots-u_{q}, & q \text { odd } \\ u_{2}-u_{1}+\cdots+u_{q}-u_{q-1}-1, & q \text { even }\end{cases}
$$

Thus for $q=1, t=p-u_{1}$, for $q=2, t=u_{2}-u_{1}-1$ and for $q=p, t=\left\lfloor\frac{1}{2}(p-1)\right\rfloor$. Assuming $12 S_{q}>N-4$ then $A_{* 1}<A_{* 2}$ and $\lambda_{* 1}$ corresponds to a stable fixed point in this multi coupling theory when $t=0$. This is possible only for $q=1$ or 2 when $u_{1}=p$ or $u_{2}=u_{1}+1$ respectively. For $S_{q}<0$ a stable fixed point arises for $t+1=p$ and $A_{* 2}<A_{* 1}$. It is then necessary to take $q=1, u_{1}=1$ and $m_{1}>4$. For $q=1, u_{1}=p$, so that only $x_{*}^{p}$ is non zero,

$$
\frac{N-4}{12 S_{q}-N+4}=\frac{(N-4)\left(4-M_{p}\right)}{8 N+(N-16) M_{p}+16}
$$

so that this positive for $M_{p}=1,2,3$ which may be realised when $p=2$ for $m_{1}=1, m_{2}=2,3$. If $q=2, u_{2}=u_{1}+1$ then

$$
\frac{N-4}{12 S_{q}-N+4}=\frac{\left(4-M_{1}\right)\left(4-M_{2}\right)}{16 M_{1}-8 M_{2}-M_{1} M_{2}-16},
$$

and for positivity we may take $M_{1}<4, M_{2}>4$. For $p=2$ and $m_{1}=1, m_{2}=2,3$ this is negative in accord with the result that there should be just one stable fixed point. It is easy to verify that these results are in accord with (7.20) when $m_{1}=1, m_{2}=2$.

\section{Conclusion}

Finding fixed points in the $\varepsilon$-expansion is hardly terra incognita, nevertheless there is as yet no mappa mundi. At one level this amounts to determining quartic, or cubic or sextic, polynomials in $\mathcal{N}$ variables invariant under all possible subgroups of $O(\mathcal{N})$. Nevertheless understanding $\mathrm{RG}$ flows between different fixed points depends to a large extent on constructing an $a$-function which decreases monotonically under RG flow. Here we considered just the lowest order expression although this can be extended, at least perturbatively, to higher orders. The discussions here suggest that fixed points are stable against RG flow to othe fixed points near a bifurcation point. However the RG flow may also lead to $a$ decreasing indefinitely, leading in unitary theories to a trivial IR limit such as when all fields are massive.

\section{Acknowledgments}

Computations in this paper have been performed with the help of FORM and also Mathematica with the package xAct.

We are grateful to Aninda Sinha, Bertrand Delamotte, Matteo Bertolini, Vladimir Bashmakov and Mikhail Kompaniets for very helpful emails. 


\section{A Bifurcations of fixed points in $6-\varepsilon$ dimensions}

The discussion of fixed points for the $O(N)$ theory may be extended to higher orders using results for the two and three loop $\beta$-functions. To three loop order the fixed points as functions of $N$ are determined by solving

$$
f(x)+g^{2} f_{2}(x)+g^{4} f_{3}(x)=0, \quad g^{2} f_{g}(x)+g^{4} f_{g 2}(x)=\frac{1}{2} \varepsilon,
$$

with $f_{g}(x), f(x)$ defined in (4.8), (4.9) and from the two and three loop $\beta$-functions

$$
\begin{aligned}
& f_{2}(x)= \frac{1}{108}\left(\left(-26 x^{3}+78 x^{2}+220 x+18\right) N+\left(97 x^{4}-6 x^{3}-157 x^{2}-90 x-134\right) x\right), \\
& f_{g 2}(x)=-\frac{1}{432}(\left.\left(11 x^{2}-132 x+86\right) N-13 x^{4}+24 x^{3}+628 x^{2}+360 x+536\right), \\
& f_{3}(x)=\frac{1}{31104}\left(\left(-1185 x^{3}+16974 x^{2}-59542 x+41544\right) N^{2}\right. \\
& \quad+\left(-9884 x^{5}-10752 x^{4}+218830 x^{3}-24408 x^{2}+109456 x+51336\right) N \quad(\text { A. } 2) \\
&\left.\quad+\left(52225 x^{6}-16806 x^{5}-4980 x^{4}-48888 x^{3}-179240 x^{2}-9000 x-125680\right) x\right) \\
&+\frac{1}{12}\left(\left(-12 x^{3}+23 x^{2}+66 x-22\right) N+7 x^{6}+6 x^{5}-12 x^{4}-24 x^{3}+8 x^{2}-36 x-4\right) x \zeta_{3} .
\end{aligned}
$$

To this order finding fixed points is equivalent to solving

$$
f(x)+\varepsilon F_{2}(x)+\varepsilon^{2} F_{3}(x)=0, \quad F_{2}(x)=\frac{f_{2}(x)}{2 f_{g}(x)}, \quad F_{3}(x)=\frac{f_{3}(x)}{4 f_{g}(x)^{2}}-\frac{f_{2}(x) f_{g 2}(x)}{4 f_{g}(x)^{3}} .
$$

For a fixed point the $\varepsilon$-expansion

$$
x_{*}=x_{1}+x_{2} \varepsilon+x_{3} \varepsilon^{2}+\mathrm{O}\left(\varepsilon^{3}\right), \quad f\left(x_{1}\right)=0,
$$

is then given by

$$
x_{2}=-\frac{F_{2}\left(x_{1}\right)}{f^{\prime}\left(x_{1}\right)}
$$

and

$$
x_{3}=-\frac{1}{f^{\prime}\left(x_{1}\right)}\left(F_{3}\left(x_{1}\right)+F_{2}^{\prime}\left(x_{1}\right) x_{2}+\frac{1}{2} f^{\prime \prime}\left(x_{1}\right) x_{2}^{2}\right) .
$$

The $\varepsilon$-expansion breaks down when two fixed points collide since then $f^{\prime}\left(x_{1}\right) \rightarrow 0$. If $x_{* \pm}$ are fixed points which coincide when $N \rightarrow N_{\text {crit }}$ then near the bifurcation point $x_{*+}+x_{*-}$ is regular but for the difference the $\varepsilon$-expansion may be modified to the form

$$
x_{*+}-x_{*-} \approx \frac{x_{1+}-x_{1-}}{\sqrt{d(N)}}\left(d(N)+d_{1}(N) \varepsilon+d_{2}(N) \varepsilon^{2}\right)^{\frac{1}{2}},
$$

where

$$
d(N)=\frac{1}{324}\left(5 N^{3}-5196 N^{2}+4848 N+464\right),
$$


is the discriminant for the cubic $f(x)$, for $d(N)>0$ there are three real roots. When $x_{1+} \rightarrow x_{1-}$ for $N \rightarrow N_{1}$ then $d\left(N_{1}\right)=0$ and $f^{\prime}\left(x_{1+}\right) \sim-f^{\prime}\left(x_{1-}\right) \rightarrow 0$. By comparing with the $\varepsilon$-expansion

$$
d_{1}(N)=2 d(N) \frac{x_{2+}-x_{2-}}{x_{1+}-x_{1-}}, \quad d_{2}(N)=2 d(N) \frac{x_{3+}-x_{3-}}{x_{1+}-x_{1-}}+\frac{d_{1}(N)^{2}}{4 d(N)} .
$$

Crucially $d_{1}(N), d_{2}(N)$ are finite as $N \rightarrow N_{1}$, although the two terms in $d_{2}(N)$ separately have poles. Hence

$$
N_{\text {crit }}=N_{1}+N_{2} \varepsilon+N_{3} \varepsilon^{2}+\mathrm{O}\left(\varepsilon^{3}\right), \quad d\left(N_{1}\right)=0
$$

where

$$
N_{2}=-d_{1}\left(N_{1}\right) / d^{\prime}\left(N_{1}\right), \quad N_{3}=-\left(d_{2}\left(N_{1}\right)+d_{1}^{\prime}\left(N_{1}\right) N_{2}+\frac{1}{2} d^{\prime \prime}\left(N_{1}\right) N_{2}^{2}\right) / d^{\prime}\left(N_{1}\right) .
$$

For the three roots of $d(N)=0$ the above formalism gives

$$
\begin{aligned}
& N_{\text {crit }}=1038.266-609.840 \varepsilon-364.173 \varepsilon^{2}, \\
& N_{\text {crit }}=1.021453+0.0325306 \varepsilon-0.0016301 \varepsilon^{2}, \\
& N_{\text {crit }}=-0.0875026+0.3472646 \varepsilon-0.8827372 \varepsilon^{2} .
\end{aligned}
$$

The results agree precisely with [23]. Correspondingly, extending (4.10),

$$
\begin{aligned}
& x_{* \text { crit }}=8.74532-0.125786 \varepsilon+0.93833 \varepsilon^{2}, \\
& x_{* \text { crit }}=1.103394+0.0676068 \varepsilon-0.022110 \varepsilon^{2}, \\
& x_{* \text { crit }}=-0.24872+0.70563 \varepsilon^{2}-1.60777 \varepsilon^{2} .
\end{aligned}
$$

\section{B Alternative fixed points in $4-\varepsilon$ dimensions}

We here discuss perturbations of the $O(N)$ theory by operators $\frac{1}{4} \phi^{2} d_{i j} \phi^{i} \phi^{j}$ with $d_{i j}$ symmetric and traceless. At the $O(N)$ fixed point this is not relevant but we find new fixed points by considering a potential

$$
V(\phi)=\frac{1}{8} \lambda\left(\phi^{2}\right)^{2}+\frac{1}{4} g \phi^{2} d_{i j} \phi^{i} \phi^{j}+\frac{1}{8} h\left(d_{i j} \phi^{i} \phi^{j}\right)^{2},
$$

where $d_{i j}$ satisfies (4.2) to ensure a closed RG flow. The one loop $\beta$-functions are then

$$
\begin{aligned}
& \beta_{\lambda}=-\varepsilon \lambda+(N+8) \lambda^{2}+(N+16) g^{2}+4(\lambda+h+b g) h, \\
& \beta_{g}=-\varepsilon g+(N+16) g(\lambda+h)+8 b g^{2}+6 b h^{2}+2 b(\lambda+b g) h, \\
& \beta_{h}=-\varepsilon h+\left(N+4+8 b^{2}\right) h^{2}+(N+16) g^{2}+12 \lambda h+28 b g h .
\end{aligned}
$$

A simpler form is obtained by taking $b=\alpha-1 / \alpha$ with $\alpha=\sqrt{\frac{n}{m}}, N=m+n$ and defining

$$
g_{1}=\lambda+2 \alpha g+\alpha^{2} h, \quad g_{3}=\lambda-\frac{2}{\alpha} g+\frac{1}{\alpha^{2}} h, \quad g_{2}=\lambda+\left(\alpha-\frac{1}{\alpha}\right) g-h,
$$


and with $\varphi=\delta_{+} \phi, \psi=\delta_{-} \phi$, using definitions in (4.30),

$$
V(\phi)=\frac{1}{8} g_{1}\left(\varphi^{2}\right)^{2}+\frac{1}{8} g_{3}\left(\psi^{2}\right)^{2}+\frac{1}{4} g_{2} \varphi^{2} \psi^{2},
$$

and the lowest order $\beta$-functions become

$$
\begin{aligned}
& \beta_{g_{1}}=-\varepsilon g_{1}+(m+8) g_{1}^{2}+n g_{2}^{2}, \\
& \beta_{g_{3}}=-\varepsilon g_{3}+(n+8) g_{3}^{2}+m g_{2}^{2}, \\
& \beta_{g_{2}}=-\varepsilon g_{2}+\left((m+2) g_{1}+(n+2) g_{3}\right) g_{2}+4 g_{2}^{2} .
\end{aligned}
$$

Positivity of $V$ requires $g_{1}, g_{3}>0$ and $g_{1} g_{3}>g_{2}{ }^{2}$. The theory described by (B.4) was considered previously in [76]. Two and three loop results for anomalous dimensions and $\beta$-functions are given in an ancillary file.

Under RG flow the $h$ coupling plays the crucial role in obtaining IR fixed points. This term may be expressed in terms of a symmetric traceless 4 -tensor by writing $\left(d_{i j} \phi^{i} \phi^{j}\right)^{2}=$ $d_{i j k l} \phi_{i} \phi_{j} \phi_{k} \phi_{l}+\frac{4}{N+4} \phi^{2} d_{i j} \phi^{i} \phi^{j}+\frac{2}{N+2}\left(\phi^{2}\right)^{2}$ and where $d_{i j k l} \phi_{i} \phi_{j} \phi_{k} \phi_{l}$ becomes relevant for $N>4$. Manifestly for $g_{2}=0$ there are two decoupled theories with $O(m)$ and $O(n)$ symmetry and, apart from the Gaussian fixed point, there are fixed points with $\left(g_{1 *}, g_{3 *}\right)=$ $\left(\frac{1}{m+8}, 0\right) \varepsilon,\left(0, \frac{1}{n+8}\right) \varepsilon,\left(\frac{1}{m+8}, \frac{1}{n+8}\right) \varepsilon$. The eigenvalues of the stability matrix are in each case $\left(1,-1,-\frac{6}{m+8}\right) \varepsilon,\left(1,-1,-\frac{6}{n+8}\right) \varepsilon$ and $\left(1,1, \frac{(m+2)(n+2)-36}{(m+8)(n+8)}\right) \varepsilon$. The last case is then stable for $m=1, n>10, m=2, n>7, m=3, n>5, m=4, n>4$ and also with $m \leftrightarrow n$. For these fixed points the anomalous dimension matrix $\left(\gamma_{\phi}\right)_{i j}$ is no longer proportional to the unit matrix but has two eigenvalues, either zero or $\gamma_{\varphi}, \gamma_{\psi}$ for the $O(m), O(n)$ symmetric theories.

For $g_{2} \neq 0$ there is the $O(N)$ symmetric fixed point with $g_{1 *}=g_{2 *}=g_{3 *}=\frac{1}{N+8} \varepsilon$ for which the stability matrix eigenvalues are $\left(1, \frac{8}{N+8}, \frac{4-N}{N+8}\right) \varepsilon$ so that this is unstable for $N>4$. Finding other fixed points with $g_{2}$ non zero, realising a coupled theory with $O(m) \times O(n)$ symmetry, is more involved. For $n=m$ there is a symmetry $\varphi \leftrightarrow \psi$ and it is easy to see by requiring $\beta_{g_{1}}-\beta_{g_{3}}=0$ that we must take $g_{1}=g_{3}$ or $g_{1}+g_{3}=\frac{1}{m+8} \varepsilon$. For the latter there are no real fixed points for $m>1$. For the former we may recover the $O(N), N=2 m$, fixed point or $g_{1 *}=g_{3 *}=\frac{m}{2\left(m^{2}+8\right)} \varepsilon, g_{2 *}=\frac{4-m}{2\left(m^{2}+8\right)} \varepsilon$. The stability matrix eigenvalues for this case are then $\left(1, \frac{8(m-1)}{m^{2}+8},-\frac{(m-2)(m-4)}{m^{2}+8}\right) \varepsilon$, where $m=3$ is stable. For this case the anomalous dimension matrix has a single eigenvalue, to lowest order $\gamma_{\phi}=\frac{m\left(m^{2}-3 m+8\right)}{8\left(m^{2}+8\right)^{2}} \varepsilon^{2}$. This fixed point coincides with the decoupled fixed point with $O(m) \times O(m)$ symmetry when $m=4$. For $m=n$ the stable fixed points are then the $O(2 m)$ symmetric case for $m<2$, the coupled $O(m) \times O(m)$ fixed point for $2<m<4$ and the decoupled one for $m>4$.

For $g_{2} \neq 0$ and $m \neq n$ finding fixed points is simplified by letting $x=g_{1} / g_{2}, y=g_{3} / g_{2}$ and then it is sufficient to solve

$$
((m+2) x-6 y+4) y=m, \quad((n+2) y-6 x+4) x=n .
$$

The couplings at a $g_{2}$ non zero fixed point to $\mathrm{O}(\varepsilon)$ are given by

$$
g_{2 *}=\varepsilon /(4+(m+2) x+(n+2) y), \quad g_{1 *}=x g_{2 *}, \quad g_{3 *}=y g_{2 *} .
$$


The $O(N)$ solution corresponds to $x=y=1$. Defining

$$
p=(m+2)(n+2)-36,
$$

then for $p$ small there is a solution

$$
x \approx-\frac{4}{p}(n+8)-\frac{m(n+2)}{4(m+8)}-\frac{3 n}{2(n+8)}, \quad y \approx-\frac{4}{p}(m+8)-\frac{n(m+2)}{4(n+8)}-\frac{3 m}{2(m+8)},
$$

giving

$$
g_{2 *} \approx-\frac{p}{4(m+8)(n+8)} \varepsilon, \quad g_{1 *}=\frac{1}{m+8} \varepsilon+\mathrm{O}(h), \quad g_{3 *}=\frac{1}{n+8} \varepsilon+\mathrm{O}(h) .
$$

As $p \rightarrow 0$ this coincides with the decoupled $O(m) \times O(n)$ fixed point. Besides $x=y=$ 1 (B.6) has three solutions, for $m, n>1$ two are complex conjugates, one is real and matches with (B.9) as $p \rightarrow 0$. For $m \rightarrow 0, x \rightarrow-\frac{3 n}{n-16}, y \rightarrow-\frac{n-32}{3(n-16)}$ and similarly for $m \leftrightarrow n, x \leftrightarrow y$. For $m, n$ large $x \sim a, y \sim 1 / a$ where $3 a^{3}+a^{2}+\frac{n}{m}(a+3)=0$ giving $-\frac{1}{3}>a>-3$ for $0<\frac{n}{m}<\infty$ and $a=-1$ for $n=m$.

At higher orders the critical point where the decoupled and coupled theories is shifted from $p=0$. Imposing the requirement that one of the eigenvalues of the stability matrix in the decoupled theory vanishes gives

$$
p_{\text {crit }}=-48 \varepsilon+8\left(1+3 \zeta_{3}\right) \varepsilon^{2}+\frac{72\left(7+6 \zeta_{3}\right)}{m+n+16} \varepsilon^{2},
$$

or in the symmetric case $n=m$

$$
m_{\mathrm{crit}}=4-4 \varepsilon+\frac{1}{2}\left(1+7 \zeta_{3}\right) \varepsilon^{2} .
$$

The same condition ensures $g_{2}=0$ in the coupled theory.

\section{Bounds for tensor products}

The tensorial relation (5.2) necessary at lowest order for a simple two coupling renormalisable theory in four dimensions introduces two parameters $a, b$. Here we demonstrate the inequality (5.3) valid so long as $d_{i j k l}$ are real. To this end we consider the decomposition of the rank six tensor $d_{i j k p} d_{l m n p}$ into irreducible components.

A symmetric traceless tensor may be defined by

$$
S_{i j k l m n}=d_{(i j k \mid p} d_{l m n) p}-\frac{9 b}{N+8} \delta_{(i j} d_{k l m n)}-\frac{3 a}{N+4} \delta_{(i j} \delta_{k l} \delta_{m n)} .
$$

Using (5.59) and (5.61) this has the norm

$$
S_{i j k l m n} S_{i j k l m n}=\frac{1}{40} N(N+2) a\left(\frac{(N+2)(N-2)(N+14)}{(N-1)(N+4)} a+\frac{18(N+2)}{N+8} b^{2}-9 e^{u} h_{u}\right) .
$$


A mixed symmetry tensor corresponding to a $[4,2]$ Young tableaux is given by

$$
\begin{aligned}
M_{i j k l m n}= & d_{i j(k \mid p} d_{l m n) p}-d_{i(k l \mid p} d_{m n) j p} \\
& +\frac{b}{N-2}\left(\delta_{i j} d_{k l m n}-\delta_{i(k} d_{l m n) j}-\delta_{j(k} d_{l m n) i}+\delta_{(k l} d_{m n) i j}\right) \\
& +\frac{a}{N-1}\left(\delta_{i j} \delta_{(k l} \delta_{m n)}-\delta_{i(k} \delta_{l m} \delta_{n) j}\right)-\frac{5}{N+4} e^{u} w_{i j(k l)} \delta_{m n)},
\end{aligned}
$$

satisfying $M_{i j k l m n}=M_{(i j)(k l m n)}, M_{i(j k l m n)}=0$ and traceless on contraction of any pair of indices. The norm is then

$$
M_{i j k l m n} M_{i j k l m n}=\frac{5}{48} N(N+2) a\left((N+2) \frac{N-2}{N-1} a-\frac{2(N+2)}{N-2} b^{2}+\frac{N-16}{N+4} e^{u} h_{u}\right) .
$$

From (5.62) $a e^{u} h_{u}=e^{u} e^{v} a_{u v}^{\prime} \geq 0$. Setting the $w$ tensor contributions to zero positivity for $N>2$ is equivalent to (5.3). For $N=3 M_{i j k l m n}=0$ and we must take $e^{u} h_{u}=0$ so that $a=$ $4 b^{2}$ then. For $N<16$ the $w$-tensor contributions ensure a more stringent bound on $b^{2} / a$.

The 8 index tensor formed from two $d_{i j k l}$ may also be decomposed into representations of $O(N)$. The mixed symmetry $[4,4]$ representation gives the bound $a \leq 15 b^{2}$ in the absence of $w$-tensor contributions when $N=4$.

As an illustration of similar constraints we consider a similar discussion for a three index symmetric traceless tensor $d_{i j k}$ which may define a single coupling renormalisable theory in six dimensions if at one loop order it satisfies $[24,77]$

$$
d_{i k l} d_{j k l}=\alpha \delta_{i j}, \quad \alpha>0, \quad d_{i l m} d_{j m n} d_{k n l}=\beta d_{i j k} .
$$

In this case analogous symmetric traceless and mixed symmetry tensors are formed by

$$
\begin{aligned}
S_{i j k l} & =d_{(i j \mid m} d_{k l) m}-\frac{2 \alpha}{N+2} \delta_{(i j} \delta_{k l)}, \\
M_{i j k l} & =d_{i j m} d_{k l m}-d_{(i \mid k m} d_{j) l m}+\frac{\alpha}{N-1}\left(\delta_{i j} \delta_{k l}-\delta_{(i \mid k} \delta_{j) l}\right),
\end{aligned}
$$

where $M_{(i j k l)}=0, M_{i j k k}=M_{i k j k}=0$. Positivity of $S_{i j k l} S_{i j k l}, M_{i j k l} M_{i j k l}$ gives

$$
-\frac{N-2}{2(N+2)} \alpha \leq \beta \leq \frac{N-2}{N-1} \alpha
$$

where the lower and upper bounds require $S_{i j k l}=0$ and $M_{i j k l}=0$ respectively. At two loop order it is necessary that

$$
d_{i l m} d_{j n p} d_{k r s} d_{l n r} d_{m p s}=\gamma d_{i j k}
$$

where

$$
S_{i j k l}=0 \Rightarrow \gamma=-\frac{N^{2}-10 N-16}{2(N+2)^{2}} \alpha^{2}, \quad M_{i j k l}=0 \Rightarrow \gamma=\frac{N^{2}-4 N+5}{(N-1)^{2}} \alpha^{2} .
$$

Identities similar to (C.8) are required at higher order for graphs of new topology, the coefficients may be calculated for the two limiting cases in a similar fashion [78]. $M_{i j k l}=0$ is realised by taking $d_{i j k}=\sum_{\alpha} e_{i}^{\alpha} e_{j}^{\alpha} e_{k}^{\alpha}$ when $\alpha=\frac{N-1}{N+1}, \beta=\frac{N-2}{N+1}$. The case $S_{i j k l}=0$, with additional assumptions, reduces to the $F_{4}$ family considered in [79] where $N=5,8,14,26$. Other solutions with a single tensor $d_{i j k}$, satisfying (C.5), (C.8) and the various higher order extensions, are provided by the invariant symmetric tensors for $\mathrm{SU}(n), N=n^{2}-1$. 


\section{Perturbations at the hypertetrahedral fixed point}

We here analyse the scaling dimensions for $\phi^{4}$ operators at the stable fixed point obtained by taking $d_{i j k l} \phi^{i} \phi^{j} \phi^{k} \phi^{l}$ to be given by (5.48) and assuming $N>5$. It is convenient to define $\phi^{\alpha}=e_{i}^{\alpha} \phi_{i}, \partial^{\alpha}=e_{i}^{\alpha} \partial_{i}, \alpha=1, \ldots, N+1$, with $e_{i}^{\alpha}$ satisfying (5.47) so that $\sum_{\alpha} \phi^{\alpha}=\sum_{\alpha} \partial^{\alpha}=0$. Using (5.53) the anomalous dimensions to $\mathrm{O}(\varepsilon)$ at the stable fixed point for $N \geq 5$ are determined by the eigenvalues of the operator

$$
\mathcal{D}=\varepsilon \frac{1}{3(N+3)}\left(\frac{1}{2} \phi^{2} \partial^{2}+(\phi \cdot \partial)^{2}-\phi \cdot \partial+\frac{1}{2}(N+1) \sum_{\alpha} \phi^{\alpha} \phi^{\alpha} \partial^{\alpha} \partial^{\alpha}\right) .
$$

Under the action of the symmetry group $\mathcal{S}_{N+1} \phi^{4}$ operators can be decomposed into tensors $V^{\alpha \beta \gamma \delta}, V^{\alpha \beta \gamma}, V^{\alpha \beta}, \alpha \neq \beta \neq \gamma \neq \delta$, where $\sum_{\alpha} V^{\alpha \beta \gamma \delta}=\sum_{\alpha} V^{\alpha \beta \gamma}=\sum_{\alpha} V^{\alpha \beta}=$ 0 for each index, forming irreducible representations under permutations of the indices. Besides $\phi^{2}=\sum_{\alpha} \phi^{\alpha} \phi^{\alpha}$ two $\mathcal{S}_{N+1}$ singlets are relevant

$$
\phi^{3}=\sum_{\alpha} \phi^{\alpha} \phi^{\alpha} \phi^{\alpha}, \quad \phi^{4}=\sum_{\alpha} \phi^{\alpha} \phi^{\alpha} \phi^{\alpha} \phi^{\alpha} .
$$

We first define

$$
\begin{aligned}
V^{\alpha \beta \gamma \delta}= & \phi^{\alpha} \phi^{\beta} \phi^{\gamma} \phi^{\delta}+\frac{1}{N-5} \mathcal{P}_{12} \phi^{\alpha} \phi^{\alpha} \phi^{\beta} \phi^{\gamma} \\
& +\frac{1}{(N-4)(N-5)}\left(2 \mathcal{P}_{6} \phi^{\alpha} \phi^{\alpha} \phi^{\beta} \phi^{\beta}+2 \mathcal{P}_{12} \phi^{\alpha} \phi^{\alpha} \phi^{\alpha} \phi^{\beta}-\phi^{2} \mathcal{P}_{6} \phi^{\alpha} \phi^{\beta}\right) \\
& +\frac{1}{(N-3)(N-4)(N-5)}\left(6 \mathcal{P}_{4} \phi^{\alpha} \phi^{\alpha} \phi^{\alpha} \phi^{\alpha}-3 \phi^{2} \mathcal{P}_{4} \phi^{\alpha} \phi^{\alpha}-2 \phi^{3} \mathcal{P}_{4} \phi^{\alpha}\right) \\
& +\frac{3}{(N-2)(N-3)(N-4)(N-5)}\left(\phi^{2} \phi^{2}-2 \phi^{4}\right), \quad \operatorname{dim} \frac{1}{24}(N+1) N(N-1)(N-6),
\end{aligned}
$$

where $\mathcal{P}_{n}$ denotes a sum over the $n$ permutations of $\alpha, \beta, \gamma, \delta$ necessary for overall symmetry of the relevant term. This satisfies

$$
\mathcal{D} V^{\alpha \beta \gamma \delta}=\varepsilon \frac{4}{N+3} V^{\alpha \beta \gamma \delta} .
$$

For the three index case there is a symmetric tensor

$$
\begin{aligned}
V_{S}^{\alpha \beta \gamma}= & \mathcal{P}_{3} \phi^{\alpha} \phi^{\alpha} \phi^{\beta} \phi^{\gamma} \\
& +\frac{1}{N-3}\left(2 \mathcal{P}_{3} \phi^{\alpha} \phi^{\alpha} \phi^{\beta} \phi^{\beta}+2 \mathcal{P}_{6} \phi^{\alpha} \phi^{\alpha} \phi^{\alpha} \phi^{\beta}-\mathcal{P}_{3} \phi^{2} \phi^{\alpha} \phi^{\beta}\right) \\
& +\frac{1}{(N-2)(N-3)}\left(6 \mathcal{P}_{3} \phi^{\alpha} \phi^{\alpha} \phi^{\alpha} \phi^{\alpha}-3 \phi^{2} \mathcal{P}_{3} \phi^{\alpha} \phi^{\alpha}-2 \phi^{3} \mathcal{P}_{3} \phi^{\alpha}\right) \\
& +\frac{3}{(N-1)(N-2)(N-3)}\left(\left(\phi^{2}\right)^{2}-2 \phi^{4}\right), \quad \operatorname{dim} \frac{1}{6}(N+1) N(N-4),
\end{aligned}
$$

where $\mathcal{P}_{n}$ now symmetrises over $\alpha, \beta, \gamma$ and

$$
\mathcal{D} V_{S}^{\alpha \beta \gamma}=\varepsilon \frac{N+7}{3(N+3)} V_{S}^{\alpha \beta \gamma} .
$$


In addition there are mixed symmetry tensors $\left(V_{M}^{\alpha \beta \gamma}, V_{M}^{\alpha \gamma \beta}\right)$, determined by

$$
\begin{aligned}
V_{M}^{\alpha \beta \gamma}= & \left(\phi^{\alpha} \phi^{\alpha} \phi^{\beta}-\phi^{\alpha} \phi^{\beta} \phi^{\beta}\right) \phi^{\gamma} \\
& +\frac{1}{N}\left(\left(\phi^{\alpha} \phi^{\alpha}-\phi^{\beta} \phi^{\beta}\right) \phi^{\gamma} \phi^{\gamma}+\phi^{2}\left(\phi^{\alpha}-\phi^{\beta}\right) \phi^{\gamma}\right)+\frac{1}{N-2}\left(\phi^{\alpha} \phi^{\alpha} \phi^{\alpha} \phi^{\beta}-\phi^{\alpha} \phi^{\beta} \phi^{\beta} \phi^{\beta}\right) \\
& +\frac{1}{N(N-2)}\left(\left(\phi^{\alpha} \phi^{\alpha} \phi^{\alpha}-\phi^{\beta} \phi^{\beta} \phi^{\beta}\right) \phi^{\gamma}-(N-1)\left(\phi^{\alpha}-\phi^{\beta}\right) \phi^{\gamma} \phi^{\gamma} \phi^{\gamma}+\phi^{3}\left(\phi^{\alpha}-\phi^{\beta}\right)\right),
\end{aligned}
$$

with an overall dimension $\frac{1}{3}(N+1)(N-1)(N-3)$ and

$$
\mathcal{D} V_{M}^{\alpha \beta \gamma}=\varepsilon \frac{N+13}{3(N+3)} V_{M}^{\alpha \beta \gamma} .
$$

In the two index case

$$
\begin{aligned}
V_{1}^{\alpha \beta}= & \phi^{\alpha} \phi^{\alpha} \phi^{\alpha} \phi^{\beta}+\phi^{\alpha} \phi^{\beta} \phi^{\beta} \phi^{\beta} \\
& +\frac{1}{N-1}\left(2\left(\phi^{\alpha} \phi^{\alpha} \phi^{\alpha} \phi^{\alpha}+\phi^{\beta} \phi^{\beta} \phi^{\beta} \phi^{\beta}\right)-\phi^{3}\left(\phi^{\alpha}+\phi^{\beta}\right)\right)-\frac{2}{N(N-1)} \phi^{4}, \\
V_{2}^{\alpha \beta}= & \phi^{\alpha} \phi^{\alpha} \phi^{\beta} \phi^{\beta} \\
& +\frac{1}{N-1}\left(\phi^{\alpha} \phi^{\alpha} \phi^{\alpha} \phi^{\alpha}+\phi^{\beta} \phi^{\beta} \phi^{\beta} \phi^{\beta}-\phi^{2}\left(\phi^{\alpha} \phi^{\alpha}+\phi^{\beta} \phi^{\beta}\right)\right)+\frac{1}{N(N-1)}\left(\left(\phi^{2}\right)^{2}-\phi^{4}\right), \\
V_{3}^{\alpha \beta}= & \phi^{2} \phi^{\alpha} \phi^{\beta}+\frac{1}{N-1} \phi^{2}\left(\phi^{\alpha} \phi^{\alpha}+\phi^{\beta} \phi^{\beta}\right)-\frac{1}{N(N-1)}\left(\phi^{2}\right)^{2},
\end{aligned}
$$

where each have dimension $\frac{1}{2}(N+1)(N-2)$, and

$$
V_{A}^{\alpha \beta}=\phi^{\alpha} \phi^{\alpha} \phi^{\alpha} \phi^{\beta}-\phi^{\alpha} \phi^{\beta} \phi^{\beta} \phi^{\beta}+\frac{1}{N+1} \phi^{3}\left(\phi^{\alpha}-\phi^{\beta}\right), \quad \operatorname{dim} \frac{1}{2} N(N-1) .
$$

For these

$$
\begin{aligned}
\mathcal{D}\left(\begin{array}{c}
V_{1}^{\alpha \beta} \\
V_{2}^{\alpha \beta} \\
V_{3}^{\alpha \beta}
\end{array}\right) & =\varepsilon \frac{1}{3(N+3)}\left(\begin{array}{ccc}
3(N+3) & -6 & 6 \\
-4 & 2(N+5) & 0 \\
2(N+1) & 0 & 2(N+8)
\end{array}\right)\left(\begin{array}{c}
V_{1}^{\alpha \beta} \\
V_{2}^{\alpha \beta} \\
V_{3}^{\alpha \beta}
\end{array}\right), \\
\mathcal{D} V_{A}^{\alpha \beta} & =\varepsilon V_{A}^{\alpha \beta} .
\end{aligned}
$$

In addition

$$
\begin{aligned}
U_{1}^{\alpha} & =\phi^{\alpha} \phi^{\alpha} \phi^{\alpha} \phi^{\alpha}-\frac{1}{N+1} \phi^{4}, \quad U_{2}^{\alpha}=\phi^{2} \phi^{\alpha} \phi^{\alpha}-\frac{1}{N+1}\left(\phi^{2}\right)^{2}, \quad U_{3}^{\alpha}=\phi^{3} \phi^{\alpha}, \\
\mathcal{D}\left(\begin{array}{l}
U_{1}^{\alpha} \\
U_{2}^{\alpha} \\
U_{3}^{\alpha}
\end{array}\right) & =\varepsilon \frac{1}{3(N+3)}\left(\begin{array}{ccc}
6(N+1) & 6 & 0 \\
4(N+1) & 3(N+5) & -4 \\
3(N+1) & 0 & 3(N+3)
\end{array}\right)\left(\begin{array}{c}
U_{1}^{\alpha} \\
U_{2}^{\alpha} \\
U_{3}^{\alpha}
\end{array}\right),
\end{aligned}
$$

where $U_{1}^{\alpha}, U_{2}^{\alpha}, U_{3}^{\alpha}$ each have dimension $N$, and there are also two singlets

$$
\mathcal{D}\left(\begin{array}{c}
\phi^{4} \\
\left(\phi^{2}\right)^{2}
\end{array}\right)=\varepsilon \frac{1}{3(N+3)}\left(\begin{array}{cc}
6(N+1) & 6 \\
4(N+1) & 4(N+4)
\end{array}\right)\left(\begin{array}{c}
\phi^{4} \\
\left(\phi^{2}\right)^{2}
\end{array}\right) .
$$

For each perturbation then for $\kappa=\Delta-d$ to lowest order $\kappa / \varepsilon$ is given for each case in turn by $-\frac{N-1}{N+3},-\frac{2(N+1)}{3(N+3)},-\frac{2(N-2)}{3(N+3)},-\frac{N+5}{3(N+3)}, \frac{1}{6(N+3)}\left(13-N \pm \sqrt{N^{2}+22 N+25}\right), 0$, $-\frac{2}{N+3}, \frac{1}{6(N+3)}\left(3 N+9 \pm \sqrt{9 N^{2}+6 N+33}\right)$ and $1, \frac{N-5}{3(N+3)}$. The dimensions of the representations listed above add up to $\frac{1}{24} N(N+1)(N+2)(N+3)$ as expected. 


\section{E $\quad C_{T}$ for scalar theories}

For a CFT obtained at an RG fixed point a crucial observable is $C_{T}$, the coefficient of the energy momentum tensor two point function. In even dimensions this is related to particular contributions to the anomaly under Weyl recalling of the metric for a curved space background. For interacting theories the leading contributions to the anomaly have been calculated in both four and six dimensions as well as $C_{T}$ directly in both cases. Higher order terms in the perturbation expansion have not so far been determined. Here we consider some constraints obtained by matching the perturbation expansion with $O(N)$ results for large $N$ in any dimension. A similar argument is described in [80].

In four dimensions for a potential as in (3.1) then the general form for $C_{T}$ to four loops is given by, after rescaling the coupling $\lambda_{i j k l} \rightarrow 16 \pi^{2} \lambda_{i j k l}$, has the form

$$
\frac{C_{T}}{C_{T, \text { scalar }}}=N-\frac{5}{36} \lambda_{i j k l} \lambda_{i j k l}+\alpha \lambda_{i j k l} \lambda_{k l m n} \lambda_{m n i j},
$$

where $C_{T \text {,scalar }}$ is the result for a single free scalar and the four loop coefficient $\alpha$ has not been obtained directly. For the $O(N)$ symmetric theory given by (5.1) with $g=0$, so that $\lambda_{i j k l}=\lambda\left(\delta_{i j} \delta_{k l}+\delta_{i k} \delta_{j l}+\delta_{i l} \delta_{j k}\right)$, then (E.1) becomes

$$
\frac{C_{T}}{C_{T, \text { scalar }}}=N\left(1-\frac{5}{12}(N+2) \lambda^{2}+\alpha(N+2)(N+8) \lambda^{3}\right) .
$$

At the fixed point in $4-\varepsilon$ dimensions to $\mathrm{O}\left(\varepsilon^{2}\right)$, which follows from $\beta_{\lambda}$ in (5.9) and (5.23).

$$
\lambda_{*}=\frac{1}{N+8} \varepsilon+3 \frac{3 N+14}{(N+8)^{3}} \varepsilon^{2},
$$

which gives

$$
\frac{C_{T}}{C_{T, \text { scalar }}}=N\left(1-\frac{5}{12} \frac{N+2}{(N+8)^{2}} \varepsilon^{2}-\frac{5}{2} \frac{(N+2)(3 N+14)}{(N+8)^{4}} \varepsilon^{3}+\alpha \frac{N+2}{(N+8)^{2}} \varepsilon^{3}\right) .
$$

The leading $\mathrm{O}\left(N^{0}\right)$ contributions for large $N$ in [81-83] $C_{T} / C_{T, \text { scalar }}=N-\frac{5}{12} \varepsilon^{2}-\frac{7}{36} \varepsilon^{3}$, determine

$$
\alpha=-\frac{7}{36} \text {. }
$$

The result for $N=1$ then gives

$$
\frac{C_{T}}{C_{T, \text { scalar }}}=1-\frac{5}{324} \varepsilon^{2}-\frac{233}{81 \times 108} \varepsilon^{3},
$$

in accord with $[84,85]$. In an ancillary file we include results for the central charge for the theories of section 5 .

A similar approach may be adopted in six dimensions. In this case a renormalisable scalar theory, for $\mathcal{N}$ component $\phi_{i}$, with interaction $\frac{1}{6} \lambda_{i j k} \phi_{i} \phi_{j} \phi_{k}$ the general form for $C_{T}$ to three loops in perturbation theory is given by, after rescaling $\lambda_{i j k} \rightarrow(4 \pi)^{\frac{3}{2}} \lambda_{i j k}$,

$$
\frac{C_{T}}{C_{T, \text { scalar }}}=\mathcal{N}-\frac{7}{72} \lambda_{i j k} \lambda_{i j k}+\alpha \lambda_{i j k} \lambda_{i l m} \lambda_{j l n} \lambda_{k m n}+\beta \lambda_{i j k} \lambda_{i j l} \lambda_{m n k} \lambda_{m n l},
$$


where two three loop contributions are possible. For the $O(N)$ model with potential as in (4.1) with $h=0 \phi_{i} \rightarrow\left(\sigma, \varphi_{i}\right), \mathcal{N}=N+1$ and the coupling is determined by $\lambda_{000}=$ $\lambda, \lambda_{i j 0}=g \delta_{i j}$. This gives

$$
\begin{aligned}
\frac{C_{T}}{C_{T, \text { scalar }}}= & N+1-\frac{7}{72}\left(3 N g^{2}+\lambda^{2}\right) \\
& +\alpha\left(N g^{3}(3 g+4 \lambda)+\lambda^{4}\right)+\beta\left(\left(N g^{2}+\lambda^{2}\right)^{2}+4 N g^{4}\right) .
\end{aligned}
$$

This theory has a fixed point for large $N$ with $g_{*}^{2} \approx \frac{6}{N}\left(1+\frac{44}{N}-\frac{155}{3 N} \varepsilon\right) \varepsilon$ and $\lambda_{*}^{2} \approx \frac{6^{3}}{N} \varepsilon$. In the large $N$ limit to leading order in $1 / N$ corrections

$$
\frac{C_{T}}{C_{T, \text { scalar }}}=N+1-\frac{7}{4} \varepsilon+\frac{23}{288} \varepsilon^{2}+\mathrm{O}\left(\varepsilon^{3}\right) .
$$

This would require $6^{4} \beta=\frac{23}{8}$ but $\alpha$ is undetermined.

Open Access. This article is distributed under the terms of the Creative Commons Attribution License (CC-BY 4.0), which permits any use, distribution and reproduction in any medium, provided the original author(s) and source are credited.

\section{References}

[1] K.G. Wilson and M.E. Fisher, Critical exponents in 3.99 dimensions, Phys. Rev. Lett. 28 (1972) 240 [INSPIRE].

[2] A.B. Zamolodchikov, Renormalization group and perturbation theory near fixed points in two-dimensional field theory, Sov. J. Nucl. Phys. 46 (1987) 1090 [Yad. Fiz. 46 (1987) 1819] [INSPIRE].

[3] A.W.W. Ludwig and J.L. Cardy, Perturbative evaluation of the conformal anomaly at new critical points with applications to random systems, Nucl. Phys. B 285 (1987) 687 [INSPIRE].

[4] M. Lassig, Geometry of the renormalization group with an application in two-dimensions, Nucl. Phys. B 334 (1990) 652 [inSPIRE].

[5] M. Lassig, Multiple crossover phenomena and scale hopping in two-dimensions, Nucl. Phys. B 380 (1992) 601 [hep-th/9112032] [INSPIRE].

[6] M.R. Gaberdiel, A. Konechny and C. Schmidt-Colinet, Conformal perturbation theory beyond the leading order, J. Phys. A 42 (2009) 105402 [arXiv:0811.3149] [INSPIRE].

[7] R. Poghossian, Two dimensional renormalization group flows in next to leading order, JHEP 01 (2014) 167 [arXiv:1303.3015] [INSPIRE].

[8] C. Ahn and M. Stanishkov, On the renormalization group flow in two dimensional superconformal models, Nucl. Phys. B 885 (2014) 713 [arXiv:1404.7628] [INSPIRE].

[9] A. Pelissetto and E. Vicari, Critical phenomena and renormalization group theory, Phys. Rept. 368 (2002) 549 [cond-mat/0012164] [INSPIRE].

[10] L. Michel, J.-C. Toledano and P. Toledano,Landau free energies for $n=4$ and the subgroups of o(4), in Symmetries and broken symmetries in condensed matter physics, N. Boccara ed., John Wiley \& Sons Ltd, U.S.A., (1981), pg. 261. 
[11] J.-C. Toledano, L. Michel, P. Toledano and E. Brezin, Renormalization-group study of the fixed points and of their stability for phase transitions with four-component order parameters, Phys. Rev. B31 (1985) 7171.

[12] D.M. Hatch, H.T. Stokes, J.S. Kim and J.W. Felix, Selection of stable fixed points by the Toledano-Michel symmetry criterion: six-component example, Phys. Rev. B 32 (1985) 7624.

[13] J.S. Kim, D.M. Hatch and H.T. Stokes, Classification of continuous phase transitions and stable phases. I. Six-dimensional order parameters, Phys. Rev. B 33 (1986) 1774.

[14] D.M. Hatch, J.S. Kim, H.T. Stokes and J.W. Felix, Renormalization-group classification of continuous structural phase transitions induced by six-component order parameters, Phys. Rev. B 33 (1986) 6196.

[15] L. Michel, Renormalization-group fixed points of general n-vector models, Phys. Rev. B 29 (1984) 2777 [INSPIRE].

[16] E. Vicari and J. Zinn-Justin, Fixed point stability and decay of correlations, New J. Phys. 8 (2006) 321 [cond-mat/0611353] [INSPIRE].

[17] D.J. Wallace and R.K.P. Zia, Gradient properties of the renormalization group equations in multicomponent systems, Annals Phys. 92 (1975) 142 [INSPIRE].

[18] J.A. Gracey, I. Jack and C. Poole, The a-function in six dimensions, JHEP 01 (2016) 174 [arXiv: 1507.02174] [INSPIRE].

[19] H. Osborn, Weyl consistency conditions and a local renormalization group equation for general renormalizable field theories, Nucl. Phys. B 363 (1991) 486 [INSPIRE].

[20] A. Stergiou, D. Stone and L.G. Vitale, Constraints on perturbative RG flows in six dimensions, JHEP 08 (2016) 010 [arXiv: 1604.01782] [INSPIRE].

[21] S. Gukov, RG flows and bifurcations, Nucl. Phys. B 919 (2017) 583 [arXiv:1608.06638] [INSPIRE].

[22] L. Fei, S. Giombi and I.R. Klebanov, Critical $O(N)$ models in $6-\epsilon$ dimensions, Phys. Rev. D 90 (2014) 025018 [arXiv: 1404.1094] [INSPIRE].

[23] L. Fei, S. Giombi, I.R. Klebanov and G. Tarnopolsky, Three loop analysis of the critical $O(N)$ models in $6-\varepsilon$ dimensions, Phys. Rev. D 91 (2015) 045011 [arXiv:1411.1099] [INSPIRE].

[24] O.F. de Alcantara Bonfim, J.E. Kirkham and A.J. McKane, Critical exponents to order $\epsilon^{3}$ for $\phi^{3}$ models of critical phenomena in six $\epsilon$-dimensions, J. Phys. A 13 (1980) L247 [Erratum ibid. A 13 (1980) 3785] [INSPIRE].

[25] B. Grinstein, A. Stergiou, D. Stone and M. Zhong, Two-loop renormalization of multiflavor $\phi^{3}$ theory in six dimensions and the trace anomaly, Phys. Rev. D 92 (2015) 045013 [arXiv: 1504.05959] [INSPIRE].

[26] R.K.P. Zia and D.J. Wallace, On the uniqueness of $\phi^{4}$ interactions in two and three-component spin systems, J. Phys. A 8 (1975) 1089 [InSPIRE].

[27] I. Jack and H. Osborn, Analogs for the c-theorem for four-dimensional renormalizable field theories, Nucl. Phys. B 343 (1990) 647 [InSPIRE].

[28] I.F. Herbut and L. Janssen, Critical $O(2)$ and $O(3) \phi^{4}$ theories near six dimensions, Phys. Rev. D 93 (2016) 085005 [arXiv: 1510.05691] [INSPIRE].

[29] J.A. Gracey and R.M. Simms, Six dimensional Landau-Ginzburg-Wilson theory, Phys. Rev. D 95 (2017) 025029 [arXiv:1701.03618] [INSPIRE]. 
[30] A. Pelissetto, P. Rossi and E. Vicari, Large $N$ critical behavior of $O(n) \times O(m)$ spin models, Nucl. Phys. B 607 (2001) 605 [hep-th/0104024] [INSPIRE].

[31] J.A. Gracey, Chiral exponents in $O(N) \times O(m)$ spin models at $O\left(1 / N^{2}\right)$, Phys. Rev. B 66 (2002) 134402 [cond-mat/0208309] [INSPIRE].

[32] J.A. Gracey, Critical exponent omega at $O(1 / N)$ in $O(N) \times O(m)$ spin models, Nucl. Phys. B 644 (2002) 433 [hep-th/0209053] [INSPIRE].

[33] E. Brézin, J.C. Le Guillou and J. Zinn-Justin, Discussion of critical phenomena in multicomponent systems, Phys. Rev. B 10 (1974) 892 [INSPIRE].

[34] D.J. Wallace and R.K.P. Zia, Harmonic perturbations of generalized Heisenberg spin systems, J. Phys. C 8 (1975) 839.

[35] A. Aharony and M.E. Fisher, Critical behavior of magnets with dipolar interactions. I. Renormalization group near four dimensions, Phys. Rev. B 8 (1973) 3323.

[36] A. Aharony, Critical behavior of anisotropic cubic systems, Phys. Rev. B 8 (1973) 4270 [INSPIRE].

[37] D.J. Wallace, Critical behaviour of anisotropic cubic systems, J. Phys. C 6 (1973) 1390.

[38] L. Fei, S. Giombi, I.R. Klebanov and G. Tarnopolsky, Generalized F-theorem and the $\epsilon$ expansion, JHEP 12 (2015) 155 [arXiv: 1507.01960] [INSPIRE].

[39] R.K.P. Zia and D.J. Wallace, Critical behavior of the continuous $N$ component Potts model, J. Phys. A 8 (1975) 1495 [INSPIRE].

[40] N.V. Antonov, M.V. Kompaniets and N.M. Lebedev, Critical behaviour of the $O(n)-\phi^{4}$ model with an antisymmetric tensor order parameter, J. Phys. A 46 (2013) 405002 [arXiv: 1307.1991] [INSPIRE].

[41] N.V. Antonov, M.V. Kompaniets and N.M. Lebedev, Critical behavior of the $O(n) \phi^{4}$ model with an antisymmetric tensor order parameter: three-loop approximation, Theor. Math. Phys. 190 (2017) 204 [Teor. Mat. Fiz. 190 (2017) 239] [INSPIRE].

[42] H. Kawamura, Generalized chiral universality, J. Phys. Soc. Jpn. 59 (1990) 2305.

[43] S.A. Antonenko, A.I. Sokolov and K. Vaernshev, Chiral transitions in three-dimensional magnets and higher order $\epsilon$ expansions, Phys. Lett. A 208 (1995) 161.

[44] D. Mukamel and S. Krinsky, $\epsilon$-expansion analysis of some physically realizable $n \geq 4$ vector models, J. Phys. C 8 (1975) L496.

[45] N.A. Shpot, Critical behavior of the mn component field model in three-dimensions, Phys. Lett. A 133 (1988) 125.

[46] N.A. Shpot, Critical behavior of the mn component field model in three-dimensions. 2: three loop results, Phys. Lett. A 142 (1989) 474 [INSPIRE].

[47] A.I. Mudrov and K.B. Varnashev, Critical thermodynamics of three-dimensional MN component field model with cubic anisotropy from higher loop $\epsilon$-expansion, J. Phys. A 34 (2001) L347 [cond-mat/0108298] [inSPIRE].

[48] M. Stephen and J. McCauley Jr., Feynman graph expansion for tricritical exponents, Phys. Lett. A 44 (1973) 89.

[49] A.L. Lewis and F.W. Adams, Tricritical behavior in two dimensions. 2. Universal quantities from the $\epsilon$-expansion, Phys. Rev. B 18 (1978) 5099 [INSPIRE]. 
[50] P. Basu and C. Krishnan, $\epsilon$-expansions near three dimensions from conformal field theory, JHEP 11 (2015) 040 [arXiv: 1506.06616] [INSPIRE].

[51] K. Nii, Classical equation of motion and anomalous dimensions at leading order, JHEP 07 (2016) 107 [arXiv: 1605.08868] [INSPIRE].

[52] J. O'Dwyer and H. Osborn, $\epsilon$-expansion for multicritical fixed points and exact renormalisation group equations, Annals Phys. 323 (2008) 1859 [arXiv:0708.2697] [INSPIRE].

[53] R.D. Pisarski, Fixed point structure of $\phi^{6}$ in three-dimensions at large $N$, Phys. Rev. Lett. 48 (1982) 574 [INSPIRE].

[54] J. Hager and L. Schäfer, $\Theta$-point behavior of diluted polymer solutions: can one observe the universal logarithmic corrections predicted by field theory?, Phys. Rev. E 60 (1999) 2071.

[55] J.S. Hager, Six-loop renormalization group functions of $O(n)$-symmetric $\phi^{6}$-theory and $\epsilon$-expansions of tricritical exponents up to $\epsilon^{3}$, J. Phys. A 35 (2002) 2703 [InSPIRE].

[56] P.K. Townsend, Consistency of the $1 / N$ expansion for three-dimensional $\phi^{6}$ theory, Nucl. Phys. B 118 (1977) 199 [inSPIRE].

[57] T. Appelquist and U.W. Heinz, Vacuum stability in three-dimensional $O(N)$ theories, Phys. Rev. D 25 (1982) 2620 [inSPIRE].

[58] W.A. Bardeen, M. Moshe and M. Bander, Spontaneous breaking of scale invariance and the ultraviolet fixed point in $O(n)$ symmetric $\left(\phi_{3}^{6}\right)$ theory, Phys. Rev. Lett. 52 (1984) 1188 [INSPIRE].

[59] F. David, D.A. Kessler and H. Neuberger, The Bardeen-Moshe-Bander fixed point and the ultraviolet triviality of $\phi^{6}$ in three-dimensions, Phys. Rev. Lett. 53 (1984) 2071 [InSPIRE].

[60] H. Omid, G.W. Semenoff and L.C.R. Wijewardhana, Light dilaton in the large $N$ tricritical $O(N)$ model, Phys. Rev. D 94 (2016) 125017 [arXiv: 1605.00750] [INSPIRE].

[61] T. Appelquist and U.W. Heinz, Three-dimensional $O(N)$ theories at large distances, Phys. Rev. D 24 (1981) 2169 [INSPIRE].

[62] R.D. Pisarski, On the fixed points of $\phi^{6}$ in three-dimensions and $\phi^{4}$ in four-dimensions, Phys. Rev. D 28 (1983) 1554 [inSPIRE].

[63] S. Yabunaka and B. Delamotte, Surprises in $O(N)$ models: nonperturbative fixed points, large $N$ limits and multicriticality, Phys. Rev. Lett. 119 (2017) 191602 [arXiv:1707.04383] [INSPIRE].

[64] S. Giombi, I.R. Klebanov and G. Tarnopolsky, Bosonic tensor models at large $N$ and small $\epsilon$, Phys. Rev. D 96 (2017) 106014 [arXiv:1707.03866] [InSPIRE].

[65] I. Jack, D.R.T. Jones and C. Poole, Gradient flows in three dimensions, JHEP 09 (2015) 061 [arXiv: 1505.05400] [INSPIRE].

[66] I. Jack and C. Poole, $\alpha$-function in three dimensions: beyond the leading order, Phys. Rev. D 95 (2017) 025010 [arXiv: 1607.00236] [INSPIRE].

[67] D. Mukamel and S. Krinsky, Physical realizations of $n \geq 4$-component vector models. II. $\epsilon$-expansion analysis of the critical behavior, Phys. Rev. B 13 (1976) 5078.

[68] E.J. Blagoeva et al., Fluctuation-induced first-order transitions in unconventional superconductors, Phys. Rev. B 42 (1990) 6124. 
[69] A.I. Mudrov and K.B. Varnashev, Three-loop renormalization-group analysis of a complex model with stable fixed point: critical exponents up to $\epsilon^{3}$ and $\epsilon^{4}$, Phys. Rev. B 57 (1998) 3562.

[70] A.I. Mudrov and K.B. Varnashev, Stability of the three-dimensional fixed point in a model with three coupling constants from the $\epsilon$ expansion: three-loop results,

Phys. Rev. B 57 (1998) 5704.

[71] A. Cayley, On contour and slope lines, Phil. Mag. 18 (1859) 264.

[72] J.C. Maxwell, On hills and dales, Phil. Mag. 40 (1870) 421.

[73] D. Mukamel, Physical realizations of $n \geq 4$ vector models, Phys. Rev. Lett. 34 (1975) 481 [INSPIRE].

[74] L. Michel, The symmetry and renormalization group fixed points of quartic hamiltonians, in Symmetries in Particle Physics, Proceedings of a symposium celebrating Feza Gursey's sixtieth birthday, B. Bars, A. Chodos and C.-H. Tze eds., Plenum Press, U.S.A., (1984), pg. 63 [INSPIRE].

[75] G. Grinstein and D. Mukamel, Stable fixed points in models with many coupling constants, J. Phys. A 15 (1982) 233.

[76] V. Bashmakov, M. Bertolini and H. Raj, Broken current anomalous dimensions, conformal manifolds and renormalization group flows, Phys. Rev. D 95 (2017) 066011 [arXiv: 1609.09820] [INSPIRE].

[77] O.F. de Alcantara Bonfim, J.E. Kirkham and A.J. McKane, Critical exponents for the percolation problem and the Yang-Lee edge singularity, J. Phys. A 14 (1981) 2391 [INSPIRE].

[78] Y. Pang, J. Rong and N. Su, $\phi^{3}$ theory with $F_{4}$ flavor symmetry in $6-2 \epsilon$ dimensions: 3-loop renormalization and conformal bootstrap, JHEP 12 (2016) 057 [arXiv:1609.03007] [INSPIRE].

[79] P. Cvitanović, Group theory: birdtracks, Lie's, and exceptional groups, Princeton University Press, Princeton U.S.A., (2008).

[80] P. Dey, A. Kaviraj and A. Sinha, Mellin space bootstrap for global symmetry, JHEP 07 (2017) 019 [arXiv: 1612.05032] [INSPIRE].

[81] A. Petkou, Conserved currents, consistency relations and operator product expansions in the conformally invariant $O(N)$ vector model, Annals Phys. 249 (1996) 180 [hep-th/9410093] [INSPIRE].

[82] A.C. Petkou, $C_{T}$ and $C_{J}$ up to next-to-leading order in $1 / N$ in the conformally invariant $0(N)$ vector model for $2<d<4$, Phys. Lett. B 359 (1995) 101 [hep-th/9506116] [INSPIRE].

[83] K. Diab, L. Fei, S. Giombi, I.R. Klebanov and G. Tarnopolsky, On $C_{J}$ and $C_{T}$ in the Gross-Neveu and $O(N)$ models, J. Phys. A 49 (2016) 405402 [arXiv:1601.07198] [INSPIRE].

[84] R. Gopakumar, A. Kaviraj, K. Sen and A. Sinha, Conformal bootstrap in Mellin space, Phys. Rev. Lett. 118 (2017) 081601 [arXiv: 1609.00572] [INSPIRE].

[85] R. Gopakumar, A. Kaviraj, K. Sen and A. Sinha, A Mellin space approach to the conformal bootstrap, JHEP 05 (2017) 027 [arXiv: 1611.08407] [INSPIRE]. 